\title{
A tool box for implementing supersymmetric models
}

\author{
Florian Staub ${ }^{1, a}$, Thorsten Ohl ${ }^{1, b}$, Werner Porod ${ }^{1, c}$, Christian Speckner $^{2, d}$ \\ ${ }^{1}$ Institut für Theoretische Physik und Astrophysik, Universität Würzburg, \\ D-97074 Würzburg, Germany \\ ${ }^{3}$ Albert-Ludwigs-Universität Freiburg, Physikalisches Institut \\ Hermann-Herder-Straße 3, D-79104 Freiburg, Germany \\ ${ }^{a}$ Email: florian.staub@physik.uni-wuerzburg.de \\ ${ }^{b}$ Email: ohlephysik.uni-wuerzburg.de \\ ${ }^{c}$ Email: porodephysik.uni-wuerzburg . de \\ ${ }^{d}$ Email: Christian. Speckner@physik.uni-freiburg.de
}

\begin{abstract}
We present a framework for performing a comprehensive analysis of a large class of supersymmetric models, including spectrum calculation, dark matter studies and collider phenomenology. To this end, the respective model is defined in an easy and straightforward way using the Mathematica package SARAH. SARAH then generates model files for CalcHep which can be used with micrOMEGAs as well as model files for WHIZARD and $\mathrm{O}^{\prime}$ Mega. In addition, Fortran source code for SPheno is created which facilitates the determination of the particle spectrum using two-loop renormalization group equations and one-loop corrections to the masses. As an additional feature, the generated SPheno code can write out input files suitable for use with HiggsBounds to apply bounds coming from the Higgs searches to the model. Combining all program provides a closed chain from model building to phenomenology.
\end{abstract}




\section{Contents}

1. Introduction $\quad 2$

2. Building new models with SARAH 4

2.1 Overview 4

2.2 Download, installation and first evaluation 4

$\begin{array}{ll}2.3 \text { Building new models with SARAH } & 6\end{array}$

2.3.1 Model file for the MSSM 6

$\begin{array}{ll}\text { 2.3.2 Creating a model file for the NMSSM } & 10\end{array}$

$\begin{array}{lll}2.4 & \text { Calculations performed by SARAH } & 10\end{array}$

$\begin{array}{lll}2.5 & \text { Export to external programs } & 11\end{array}$

3. Spectrum calculation with SPheno 11

3.1 Introduction to SPheno 11

$\begin{array}{ll}3.2 \text { Combining SPheno and SARAH } & 12\end{array}$

$\begin{array}{lll}3.3 & \text { Features of the generated SPheno version } & 12\end{array}$

$\begin{array}{ll}\text { 3.4 Defining the properties of the generated SPheno version } & 14\end{array}$

\begin{tabular}{ll}
3.5 & Example: The input file for the MSSM \\
\hline
\end{tabular}

3.6 Generating the output 21

$\begin{array}{lll}3.7 & \text { Including Thresholds } & 22\end{array}$

3.7.1 Thresholds without gauge symmetry breaking 22

$\begin{array}{ll}\text { 3.7.2 With gauge symmetry breaking } & 23\end{array}$

3.8 Low energy SPheno version 25

3.9 Differences to SPheno3.1.4 25

3.10 Supported models and known issues 25

4. Checking Higgs constraints with HiggsBounds 26

5. Calculation of cross sections, widths and relic density using $\mathrm{Ca}$ lcHep and micrOMEGAs

5.1 Using CalcHep with SARAH and SPheno 28

$\begin{array}{lll}5.2 & \text { Relic density calculations with micrOMEGAs } & 29\end{array}$

6. Monte Carlo studies with WHIZARD 29

$\begin{array}{lll}6.1 & \text { Introduction } & 29\end{array}$

6.2 The interface between SARAH and WHIZARD 30

6.3 Generating model files and using them with WHIZARD 31

7. Parameter scans with SSP

$\begin{array}{lll}7.1 & \text { Installation and running scans } & 34\end{array}$

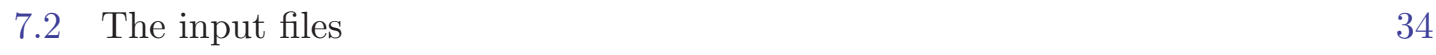

$\begin{array}{lll}7.3 & \text { Features } & 35\end{array}$ 
8. Putting the programs to work $\quad 37$

8.1 Automated setup and model implementation 37

8.2 Preparing a model by hand 38

$\begin{array}{lll}8.3 & \text { Running the chain } & 40\end{array}$

9. Conclusions 41

A. Input files for SARAH to generate a SPheno version with thresholds 41

A.1 Seesaw I 41

A.2 Implementation of a model with a gauge symmetry breaking scale in SARAH and SPheno 43

A.2.1 Summary of the model 43

$\begin{array}{lll}\text { A.2.2 Model files for SARAH } & 45\end{array}$

A.2.3 Model files for SPheno output $\quad 46$

B. Example for using SSP in the MSSM: $\left(m_{0}, M_{1 / 2}\right)$ grid scan 50

C. Validation of SPheno output $\quad 54$

C.1 Mass spectrum of the MSSM 54

C.2 Decay widths and branching ratios in the MSSM 54

C.3 Masses in Seesaw type I - III 54

C.4 Masses in the NMSSM 54

D. Validation of the WHIZARD model files $\quad 59$

\section{Introduction}

With the first collisions at the Large Hadron Collider (LHC), a new era of high energy physics has started. The LHC is designed to get new and deeper insights into the fundamental principles governing our physical world. It is not only supposed to find the last missing particle of the Standard Model (SM) of particle physics, the Higgs boson, but also to discover possible physics beyond the SM.

Despite having proven itself as a successful and precise description of all experiments in particle physics for last 30 years, the SM suffers from several theoretical shortcomings, and therefore a host of extensions of the model which lead to such new effects have been devised over the last years. Among the most prominent examples of such conceptual flaws which plague the model are the hierarchy problem $[1,2]$ and the lack of a candidate for Dark Matter.

Supersymmetry (SUSY) is arguably among the best studied extensions of the SM $[3,4$, 5] which have been developed to cure these problems. Already the minimal, supersymmetric extension of the SM, the MSSM (Minimal Supersymmetric Standard Model) [6, 7, 8], solves the hierarchy problem, is capable of providing a dark matter candidate [9, 10], leads to gauge coupling unification $[11,12,13,14]$ and relates electroweak symmetry breaking to 
the large top mass $[15,16]$. Because of these appealing features, the MSSM was extensively studied in the last decades and many software tools were developed for that purpose.

However, the MSSM might also not be the final answer. One reason is that the MSSM like the SM can't explain neutrino masses $[17,18,19,20]$. In priniple one can find for every SM extension designed to explain the observed neutrino data a supersymmetric version. The most popular among them are the various seesaw models [21, 22, 23, 24, 25, 26] leading to an effective dim-5 operator generating Majorana masses for the neutrinos [27, 28, 29]. Moreover, there is an intrinsic supersymmetric mechanism to explain neutrino data, namely the breaking of R-parity via Lepton number violating interactions [30, 31, 32, 33]. Another reason is the strong CP problem which is also still present in the MSSM. One way to solve this is to postulate an additional $U(1)$ group as proposed by Peccei and Quinn [34]. In SUSY, this would lead to the presence of a pseudo scalar Axion, the scalar Saxion and the fermionic Axino [35]. Another intrinsic problem of the MSSM is the so called $\mu$ problem: above the breaking scale, the $\mu$ parameter in the superpotential of the MSSM is the only dimensionful parameter in the model. The natural scale of that parameter would therefore be either of the GUT scale or exactly 0 if it is forbidden by symmetry. However, we know that it must be of order of the electroweak scale to explain precision data [36] and to fullfill the existing bounds from collider searches [37]. One idea to solve the $\mu$ problem is to create an effective $\mu$ term just after SUSY breaking like in the NMSSM [38].

Of course, this list can be still extended, and over the years, people have come up with many different ways of modifying and extending the MSSM. However, the way from the first idea about a SUSY model to numerical results is normally long and exhaustive: all analytical expressions for masses, vertices and renormalization group equations have to derived. Code has to be generated to calculate the numerical values for the masses and, if the results are to be reasonable, to add loop corrections. Before the existing software tools to calculate widths or cross sections as well as perform Monte Carlo studied can be used, the new model has to be implemented. That demands not only a good knowledge about the different programs but is also a very time consuming task.

In this paper, we present a framework which covers all of the aforementioned steps in an automatized way and which goes also further. Based on SARAH [39, 40, 41], a tool chain can be created for a large variety of SUSY models which covers the spectrum calculation with SPheno [42, 43], the calculation of cross sections with CalcHep [44, 45] and the determination of the dark matter relic density using micromeGAs [46], the analysis of constraints coming from the Higgs searches via HiggsBounds [47, 48] and Monte Carlo simulations of collider observables with WHIZARD $[49,50]$. The basic idea is that the user can implement the model in SARAH in an intuitive and fast way. Afterwards, SARAH generates Fortran source code for SPheno as well as model files for CalcHep, O' Mega and WHIZARD. Since the implementation of the model in all of the programs is based on the one implementation in SARAH, the conventions are the same for all programs, greatly simplifying the transfer of information between the different tools. For example, the spectrum file of SPheno can be directly used by micrOMEGAs to calculate the relic density. In addition, the new SPheno modules include the possibility to write input file for HiggsBounds. 
We start with an introduction to SARAH in sec. 2, demonstrating how models can be implemented and what information can be derived by SARAH. In sec. 3, we discuss the details of the Fortran output of SARAH for SPheno, and in sec. 4, we show how HiggsBounds can be applied to the model. Afterwards, the link to CalcHep and micrOMEGAs is discussed in sec. 5, and the output for $\mathrm{O}^{\prime}$ Mega and WHIZARD is presented in sec. 6. Finally, we introduce the package SSP which is designed for performing parameter scans using the tools supported by SARAH in sec. 7 before explaining all steps necessary to combine SARAH and SSP together with those programs into a closed tool chain in sec. 8.

\section{Building new models with SARAH}

\subsection{Overview}

SARAH is a package for Mathematica version 5.2 or higher and has been designed to handle every $N=1$ SUSY theory with an arbitrary direct product of $S U(n)$ and/or $U(1)$ factors as gauge group. The chiral superfields can transform under arbitrary, irreducible representations with regard to this gauge group, and all possible renormalizable superpotential terms are supported. There are no restrictions on either the number of gauge group factors, the number of chiral superfields or the number of superpotential terms. Furthermore, any number of symmetry breakings or field rotations is allowed. A schematic picture of the different steps performed by SARAH is shown in Fig. 1.

\subsection{Download, installation and first evaluation}

SARAH can be downloaded from

http://projects.hepforge.org/sarah/

The package should be extracted to the application directory of Mathematica,

\$HOME/.Mathematica/Applications/

on a Unix system or

[Mathematica-Directory] \AddOns \Applications \

in a Windows environment (\$HOME and [Mathematica-Directory] should be substituted with the home and Mathematica installation directories respectively).

Initially, the package itself consists of three directories: the directory Package contains all Mathematica package files, while in the directory Models the definitions of the different models are located. The third directory LaTeX contains $\mathrm{LT}_{\mathrm{E}} \mathrm{X}$ packages which are needed for the appropriate output. During execution, a fourth directory called out put is generated by SARAH where the results of the different calculations as well as the model files for the diagram calculators are stored.

A comprehensive manual (sarah.pdf) is included in the package archive and can also be found on the web page and on the arXiv [39]. In addition, a file (models.pdf) with information about all models delivered with the package is part of the archive. Furthermore, a file with a short introduction to the main commands is included (Readme.txt) as well as an example illustrating their use (Example.nb). 


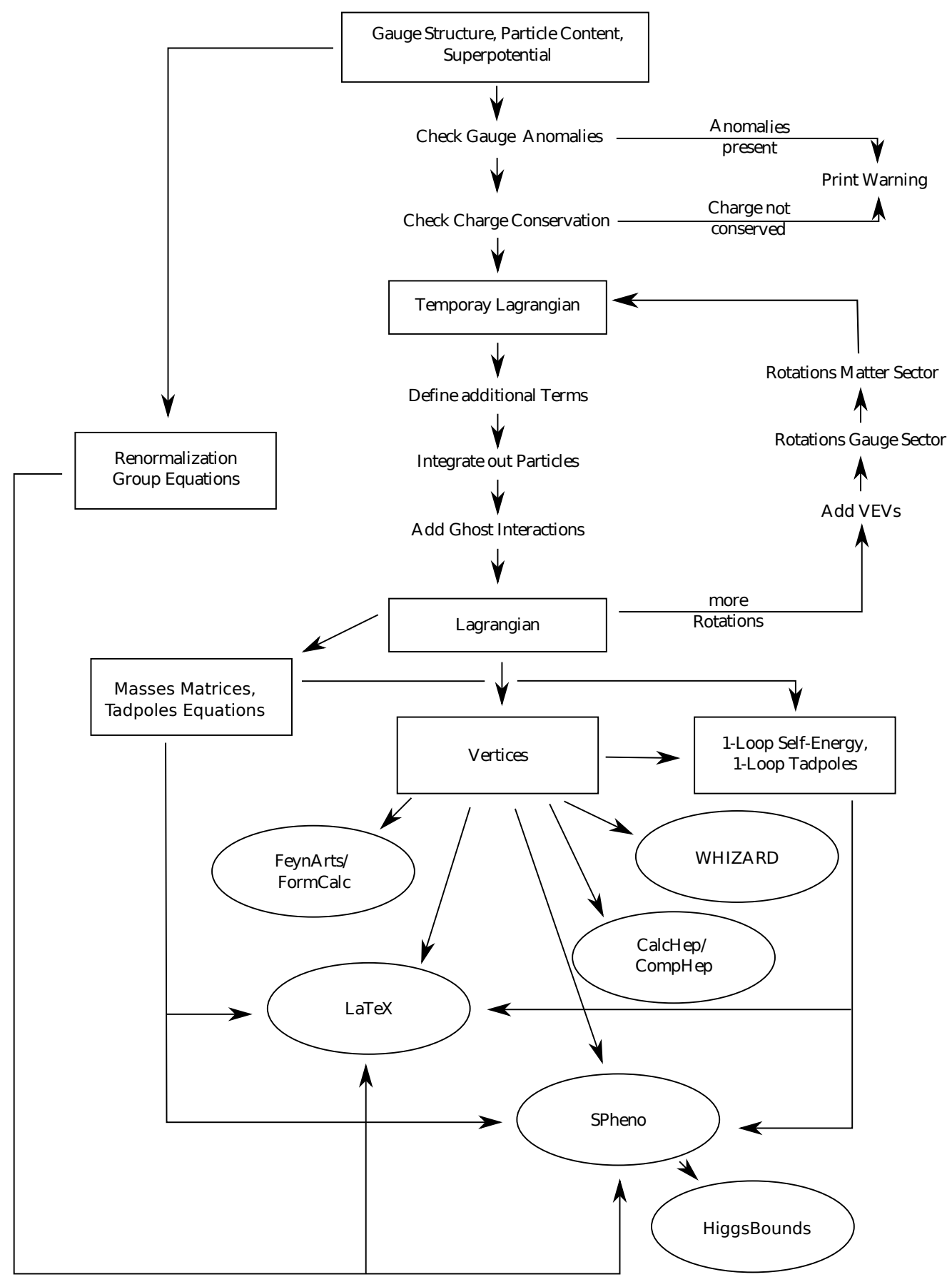

Figure 1: Schematic graph of the different steps performed by SARAH. The user has access to the calculated information shown in boxes. The ellipses show the output which can be created by SARAH.

After the installation, the package is loaded in Mathematica via

In [1]: <<"sarah-3.0/SARAH.m"

Subsequently, the model is initialized by

\section{In [2]: Start ["Modelname"];}

where Model name is the name of the corresponding model file. As an example, the command would read 
In [2]: Start [ "MSSM"] ;

for the minimal supersymmetric standard model and

In [2]: Start ["NMSSM", "CKM"] ;

for the next-to-minimal supersymmetric model in CKM basis.

\subsection{Building new models with SARAH}

As an example of how new models can be defined in SARAH, we briefly go through the existing MSSM model file and then show how this model can be augmented to obtain an implementation of the NMSSM (see [38] and references therein).

\subsubsection{Model file for the MSSM}

In the following, we showcase the different components of the MSSM implementation in SARAH (see [41] for a summary of our conventions).

1. First, give a string as internal name for the model. Make sure to use only numbers and letters and no spaces, because this name is later on used to identify the model in SPheno or CalcHep.

ModelName = "MSSM" ;

ModelNameLaTeX ="MSSM";

ModelNameLaTeX provides much more freedom: any symbol and spaces can be used and even $\mathrm{LT}_{\mathrm{E}} \mathrm{X}$ syntax is supported.

2. The gauge sector is $U(1) \times S U(2) \times S U(3)$ and is defined by declaring the corresponding vector superfields.

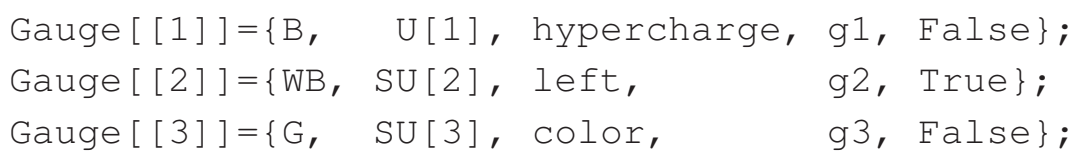

First, the name of the vector superfield is given. The second entry defines the dimension of the group, the third is the name of the gauge group and the forth gives the name of the corresponding gauge coupling. If the last entry is set to True, the sum over the group indices is expanded and the component fields are distinguished; otherwise, the sum is left implicit. In the above example, the color charges are written as indices, while the sum over the isospin multiplets is expanded.

Note that SARAH automatically adds a soft-breaking gaugino mass for every vector superfield.

3. The next step is to define the matter sector. That's done by the array Fields. The conventions are the following. First, the root of the names for the component fields is given (e.g. X): the derived names of the fermionic components start with $F$ in front (i.e. FX), while for scalars a $S$ is used (i.e. SX). At second position the number 
of generations is defined and the third entry is the name of the entire superfield. The remaining entries are the transformation properties with respect to the different gauge groups.

Using these conventions, the doublet superfields $\hat{q}, \hat{l}, \hat{H}_{d}, \hat{H}_{u}$ are added by

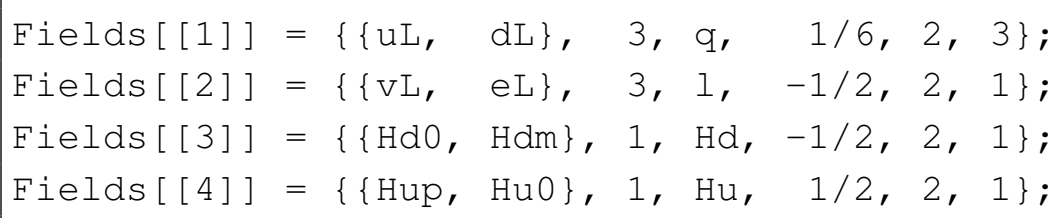

While for the singlet superfields $\hat{d}^{c}, \hat{u}^{c}, \hat{e}^{c}$

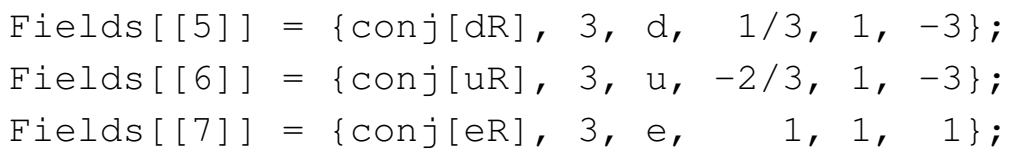

is used.

Note that for scalars SARAH also adds the soft masses automatically.

4. The superpotential of the MSSM is

$$
W=\hat{u}^{c} Y_{u} \hat{q} \hat{H}_{u}-\hat{d}^{c} Y_{d} \hat{q} \hat{H}_{d}-\hat{e}^{c} Y_{e} \hat{l} \hat{H}_{d}+\mu \hat{H}_{u} \hat{H}_{d}
$$

and represented in SARAH by

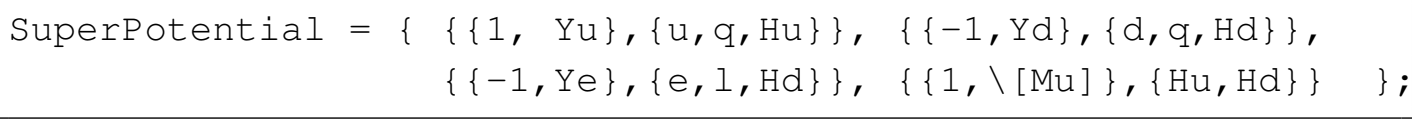

5. There are two different sets of eigenstates: the gauge eigenstates before EWSB and the mass eigenstates after EWSB. The internal names are

NameOfStates $=\{$ GaugeES, EWSB $\}$;

6. The gauge fixing terms for the unbroken gauge groups are

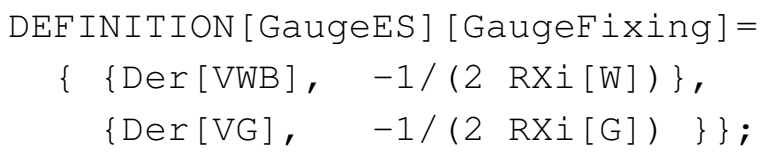

This corresponds to

$$
\mathcal{L}_{G F}=-\frac{1}{2 \xi_{W}}\left|\partial_{\mu} W^{\mu, i}\right|^{2}-\frac{1}{2 \xi_{g}}\left|\partial_{\mu} g^{\mu, i}\right|^{2}
$$

The gauge fixing terms are used for the calculation of the Ghost interactions in general $R_{\xi}$ gauge. These are used to write the vertices for FeynArts depending on gauge fixing constants, while the CalcHep output is restricted to unitary and 't Hooft gauge. Internal computations like those of the one-loop self-energies are performed in 't Hooft gauge by SARAH. 
7. The vector bosons and gauginos rotate after EWSB as follows

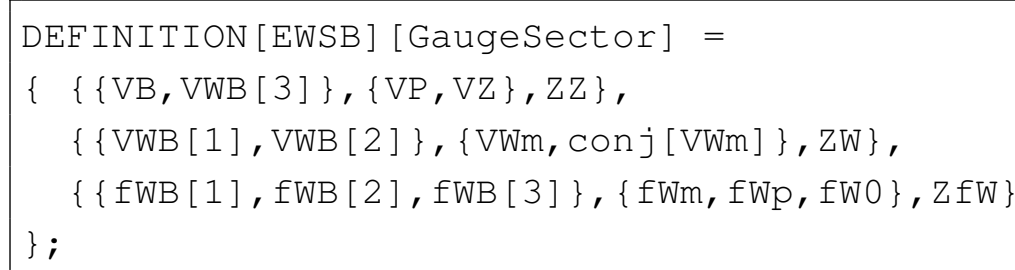

This encodes the common mixing of vector bosons and gauginos after EWSB

$$
\left(\begin{array}{c}
B \\
W^{3}
\end{array}\right)=Z^{\gamma Z}\left(\begin{array}{c}
\gamma \\
Z
\end{array}\right),\left(\begin{array}{c}
W^{1} \\
W^{2}
\end{array}\right)=Z^{W}\left(\begin{array}{c}
W^{-} \\
\left(W^{-}\right)^{*}
\end{array}\right),\left(\begin{array}{c}
\lambda_{\tilde{W}, 1} \\
\lambda_{\tilde{W}, 2} \\
\lambda_{\tilde{W}, 3}
\end{array}\right)=Z^{\tilde{W}}\left(\begin{array}{c}
\tilde{W}^{-} \\
\tilde{W}^{+} \\
\tilde{W}^{0}
\end{array}\right)
$$

The mixing matrices can easily be parameterized in SARAH to have the standard form

$$
Z^{\gamma Z}=\left(\begin{array}{cc}
\cos \Theta_{W} & -\sin \Theta_{W} \\
\sin \Theta_{W} & \cos \Theta_{W}
\end{array}\right), Z^{W}=\left(\begin{array}{cc}
\frac{1}{\sqrt{2}} & \frac{1}{\sqrt{2}} \\
-i \frac{1}{\sqrt{2}} & i \frac{1}{\sqrt{2}}
\end{array}\right), Z^{\tilde{W}}=\left(\begin{array}{ccc}
\frac{1}{\sqrt{2}} & \frac{1}{\sqrt{2}} & 0 \\
-i \frac{1}{\sqrt{2}} & i \frac{1}{\sqrt{2}} & 0 \\
0 & 0 & 1
\end{array}\right)
$$

8. The neutral components of the scalar Higgs receive vacuum expectation values (VEVs) $v_{d} / v_{u}$ and split into scalar and pseudo scalar components

$$
H_{d}^{0}=\frac{1}{\sqrt{2}}\left(v_{d}+i \sigma_{d}+\phi_{d}\right), \quad H_{u}^{0}=\frac{1}{\sqrt{2}}\left(v_{u}+i \sigma_{u}+\phi_{u}\right)
$$

This is encoded in SARAH by

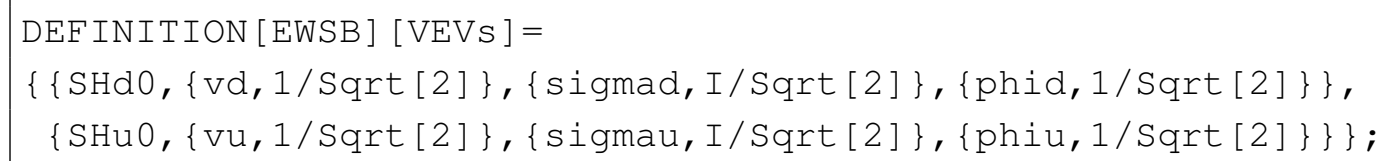

9. After EWSB the particles to new mass eigenstates

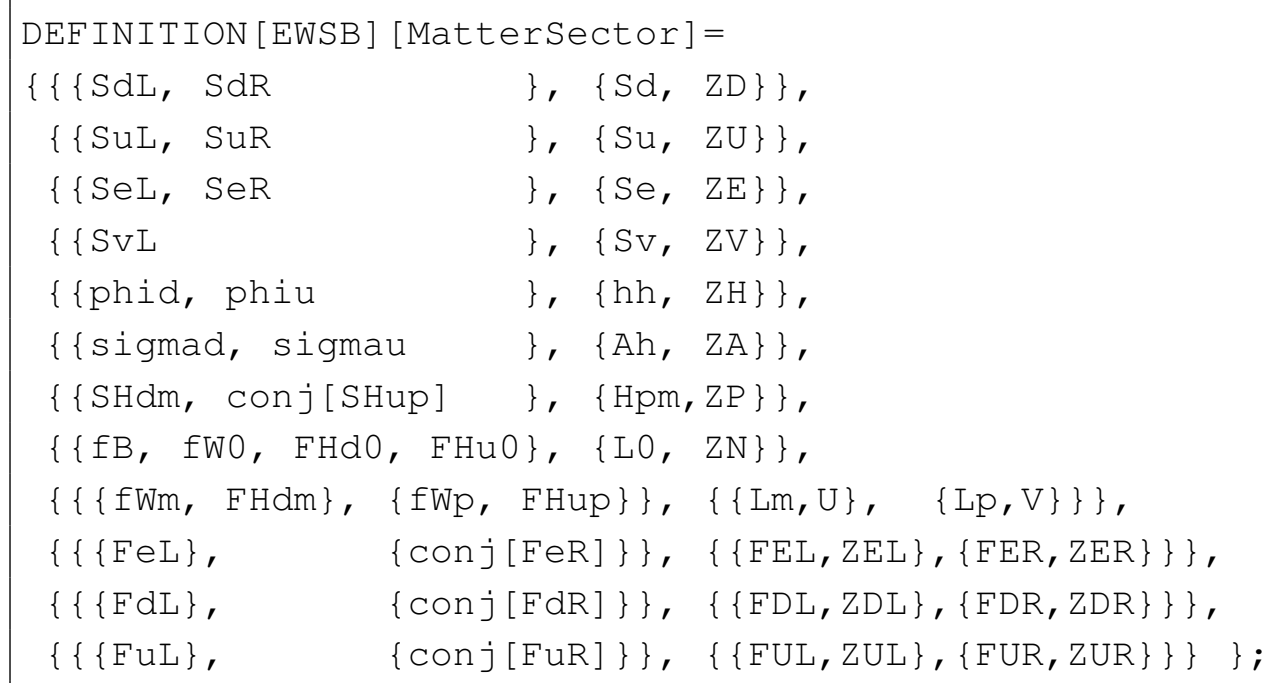


This defines the mixings to the mass eigenstates: first, a list with gauge eigenstates is given, followed by the name of the new mass eigenstates and the mixing matrix. Hence, the first line is interpreted as

$$
\tilde{d}_{L, i \alpha}=\sum_{j=1}^{3} Z_{j i}^{D, *} \tilde{d}_{j \alpha}, \quad \tilde{d}_{R, i \alpha}=\sum_{j=1}^{3} Z_{j+3 i}^{D, *} \tilde{d}_{j \alpha}
$$

while the 8 th line defines the mixing in the chargino sector

$$
\tilde{W}^{-}=\sum_{j} U_{j 1}^{*} \chi_{j}^{-}, \quad \tilde{H}_{d}^{-}=\sum_{j} U_{j 2}^{*} \chi_{j}^{-}, \quad \tilde{W}^{+}=\sum_{j} V_{1 j}^{*} \chi_{j}^{+}, \quad \tilde{H}_{u}^{+}=\sum_{j} V_{2 j}^{*} \chi_{j}^{+}
$$

10. The new gauge fixing terms after EWSB are

$$
\begin{aligned}
\mathcal{L}_{G F}= & -\frac{1}{2 \xi_{\gamma}}\left(\partial^{\mu} \gamma_{\mu}\right)^{2}-\frac{1}{2 \xi_{Z}}\left(\partial^{\mu} Z_{\mu}-\xi_{Z} M_{Z} G^{0}\right)^{2} \\
& -\frac{1}{\xi_{W^{-}}}\left|\partial^{\mu} W_{\mu}^{-}+i \xi_{W^{-}} M_{W} G^{-}\right|^{2}-\frac{1}{2 \xi_{g}}\left|\partial_{\mu} g^{\mu, i}\right|^{2} .
\end{aligned}
$$

That reads in SARAH

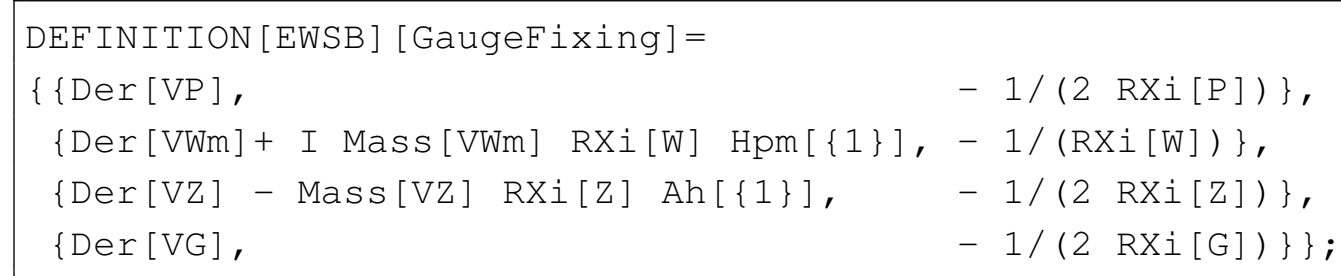

Based on this definition, $A_{1}^{0}$ and $H_{1}^{ \pm}$are interpreted in all calculations as Goldstone bosons.

11. No particles should be integrated out or deleted

Integrateout $=\{\}$;

DeleteParticles $=\{\}$;

12. The Dirac spinors for the mass eigenstates are

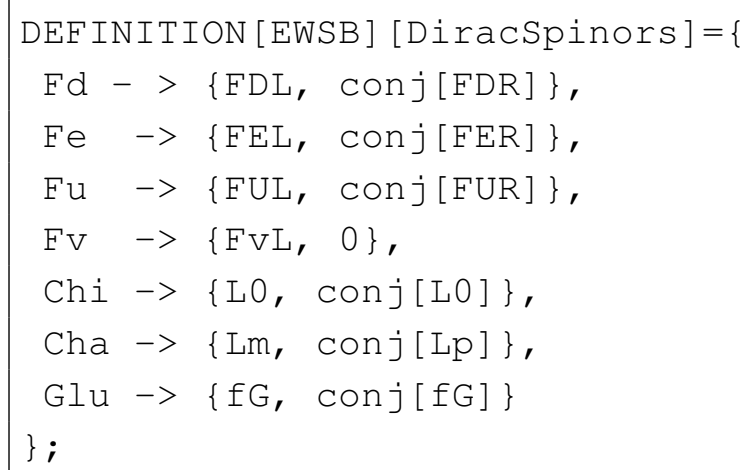


That leads to the replacements

$$
d \rightarrow\left(\begin{array}{c}
d_{L} \\
d_{R}
\end{array}\right), \ldots, \tilde{\chi}^{-} \rightarrow\left(\begin{array}{c}
\lambda^{-} \\
\left(\lambda^{+}\right)^{*}
\end{array}\right), \tilde{g} \rightarrow\left(\begin{array}{c}
\lambda_{g} \\
\lambda_{g}^{*}
\end{array}\right)
$$

when going from four- to two-component formalism.

\subsubsection{Creating a model file for the NMSSM}

Only a few changes are necessary to turn the above MSSM model definition into a fullfledged implementation of the NMSSM:

1. Add a gauge singlet superfield

$$
\text { Fields [ [8] ] }=\{\mathrm{SR}, 1, \mathrm{~s}, \quad 0,1,1\} \text {; }
$$

2. Change the superpotential

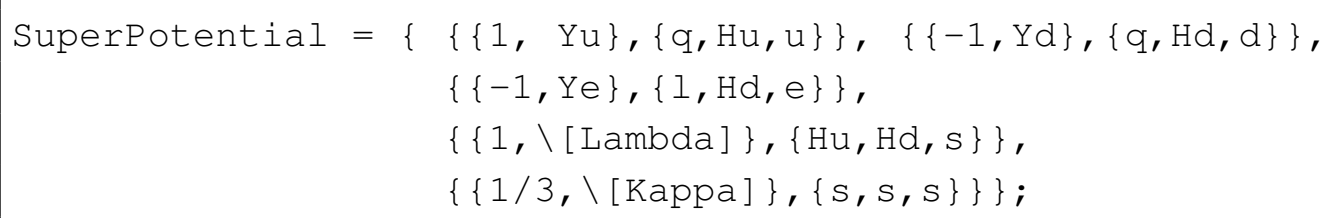

3. Give a VEV to the scalar component of the gauge singlet

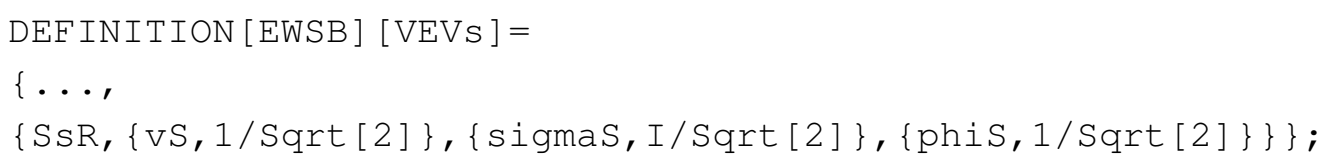

4. Mix the scalar part of the gauge singlet with the Higgs and the fermionic part with the neutralinos

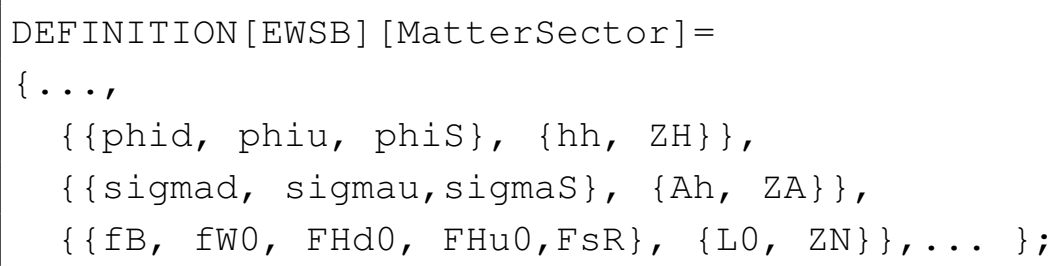

\subsection{Calculations performed by SARAH}

When a model is initialized using the Start command, it is first checked for gauge anomalies and charge conservation. If any of those checks fail, a warning is printed. Afterwards, the calculation of the complete Lagrangian at tree-level begins, and several tree-level results can be obtained after it has finished.

Masses and tadpole equations The masses and tadpole equations are derived automatically during the evaluation of a model. The user has access to both pieces of information through the command MassMatrix [Particle] for the mass matrix of Particle and TadpoleEquation [VEV] for the tadpole equation of the corresponding field. 
Vertices SARAH can be instructed to either calculate all vertices present in the model or to extract only those for specific combinations of external particles. The latter task is performed by

Vertex [ $\{$ Particles $\}$, Options ] ;

(the argument of this function being a list of external particles), while all vertices for a set of eigenstates can be calculated via

MakeVertexList [Eigenstates, Options];

This searches for all possible interactions present in the Lagrangian and creates lists for the generic subclasses of interactions. For more details about the calculation of a supersymmetric Lagrangian and the conventions for extracting the vertices we refer to the appendix of the SARAH manual [39].

Renormalization group equations SARAH calculates the RGEs for the parameters of the superpotential, the soft-breaking terms, the gauge couplings at one- and two-loop level and the VEVs. This is done by using the generic formulas of [51]. In addition, to handle the case of several, abelian gauge groups, the rules given in [52] are implemented. The calculation of the RGEs can be started after the initialization of a model via

CalcRGEs [Options] ;

Loop Corrections SARAH calculates the analytical expressions for the one-loop corrections to the one- and two-point functions (tadpoles and self energies of all particles). These calculations are performed in $\overline{\mathrm{DR}}$-scheme using the 't Hooft gauge. This is a generalization of the calculations for the MSSM presented in [53]. The command to start the calculation is

CalcLoopCorrections [Eigenstates];

\subsection{Export to external programs}

SARAH can export the information derived from the model definition in a form suitable for use with a number of external programs, among them FeynArts/FormCalc, CalcHep/CompHep, WHIZARD/O'Mega, SPheno and also plain $\mathrm{IAT}_{\mathrm{E} X} \mathrm{X}$. In the next sections, we proceed with a detailed discussion of this functionality and demonstrate how it can be used in order to facilitate a comprehensive analysis of new models.

\section{Spectrum calculation with SPheno}

\subsection{Introduction to SPheno}

SPheno $[42,43]$ is a F95 program designed for the precise calculation of the masses of supersymmetric particles. For this the formulas of [53] for the 1-loop masses have been extended to account for the flavour structures, e.g. to calculate the 1-loop corrected $6 \times 6$ mass matrices for squarks and charged leptons and the 1-loop corrected $3 \times 3$ mass matrix 
for sneutrinos $[54,55]$. Here the complete one-loop contributions including general flavour mixing and general CP-phases are taken into account. In addition, the dominant twoloop contributions for the Higgs boson masses are included based on [56, 57, 58, 59, 60] Moreover, the decay rates for 2- and 3-body decays of supersymmetric particles and Higgs bosons are calculated. The current version of SPheno can be downloaded from

http://projects.hepforge.org/spheno/

\subsection{Combining SPheno and SARAH}

SARAH is based on Mathematica and therefore it is not sensible to do exhaustive numerical calculations in SARAH's native environment. As opposed to that, SPheno provides fast numerically routines for the evaluation of the RGEs, calculating the phase space of 2- and 3-body decays as well as Passarino Veltman integrals and much more. Since these routines are model independent, they can be used for all SUSY models implemented in SARAH.

Our approach for combining all advantages of SPheno and SARAH in order to create a very efficient and easy way from model building to numerical results is depicted in Fig. 2: the model is defined in SARAH in the usual way. SARAH calculates all analytical expressions needed for a complete analysis of the model. This information is exported to Fortran code in a way suitable for inclusion in SPheno. This generates a fully functional version of SPheno for the new model without any need to change the source code by hand.

The user has control over the properties of the generated SPheno version by means of a special input file for SARAH. First, it is possible to define the free parameters of the model. Those build later on the Block MINPAR in the LesHouches input file. Second, the boundary conditions at the GUT-, SUSY- and electroweak scales as well as at possible threshold scales can be set. Third, the parameters which are to be fixed by the solutions of the tadpoles equations can be defined. An approximate solution to the tadpoles can also be given, if there isn't an analytic one.

SARAH produces replacements for all model dependent files of SPheno. These files have to be copied to a new subdirectory of the SPheno directory. A Makefile for compiling the new model afterwards as well as a template for a LesHouches input file are written by SARAH.

The command to automatically calculate all necessary information like vertices and RGEs and generate the source code is

In [3] : MakeSPheno [Options];

The name of the SPheno specific input file of SARAH can be given as an option. This offers the possibility to easily create SPheno versions for the same model with changed boundary conditions or another set of free parameters.

\subsection{Features of the generated SPheno version}

Calculation of the mass spectrum The SPheno version generated by SARAH calculates the complete mass spectrum using 2-loop RGEs and 1-loop corrections to the masses, including the full momentum dependence of all loop integrals. In addition, for MSSM-like 
Higgs sectors, the known two loop corrections to the Higgs masses and tadpoles can be included. All calculations are performed with the most general flavor structure and allow for the inclusion of CP phases.

The calculation of the mass spectrum happens in an iterative way: the gauge and Yukawa couplings are fitted at $M_{Z}$. Afterwards, a run to the GUT scale is performed and the boundary conditions are enforced. The parameters are than evolved down again to the SUSY scale and the mass spectrum is calculated. These steps are iterated until the all masses have converged to a given relative precision, by default $10^{-4}$.

The routines of SARAH for calculating the gauge and Yukawa couplings closely follow the procedures described in [42]. The values for the Yukawa couplings giving mass to the SM fermions and the gauge couplings are determined at the scale $M_{Z}$ based on the measured values of the quark and lepton masses, the mass of the $Z$-boson $M_{Z}$, the Fermi constant $G_{F}$ and the electromagnetic coupling in the Thompson limit $\alpha_{e m}(0)$. The 1-loop corrections to the mass of W- and Z-boson as well as the SUSY contributions to muon-decay are taken into account into the calculation. In addition, we include the complete 1-loop corrections to the self-energies of SM fermions [53] and re-sum the $\tan \beta$ enhanced terms in the calculation of the Yukawa couplings of the $b$-quark and the $\tau$-lepton as described in [42]. The vacuum expectation values $v_{d}$ and $v_{u}$ are calculated with respect to the given value of $\tan \beta$ at $M_{Z}$.

SUSY scale input It is also possible to define the full set of free parameters (i.e. gauge couplings, VEVs, superpotential and soft-breaking parameters) of the model at a specific scale without RGE running. These parameters are afterward used to calculate the loop corrected mass spectrum and the decays. To use this option with SPheno, the LesHouches input file ${ }^{1}$ must contain

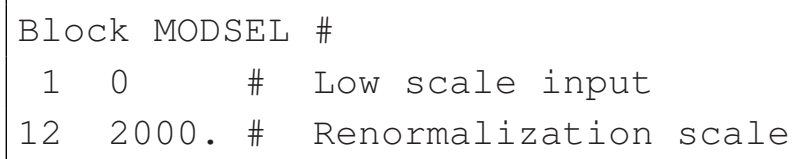

In this example all parameters are declared to be renormalized at $2 \mathrm{TeV}$. If the no explicit renormalization scale is defined by the flag $12,1 \mathrm{TeV}$ is used. In addition, the numerical values of all parameters have to be given in the LesHouches input file, e.g.

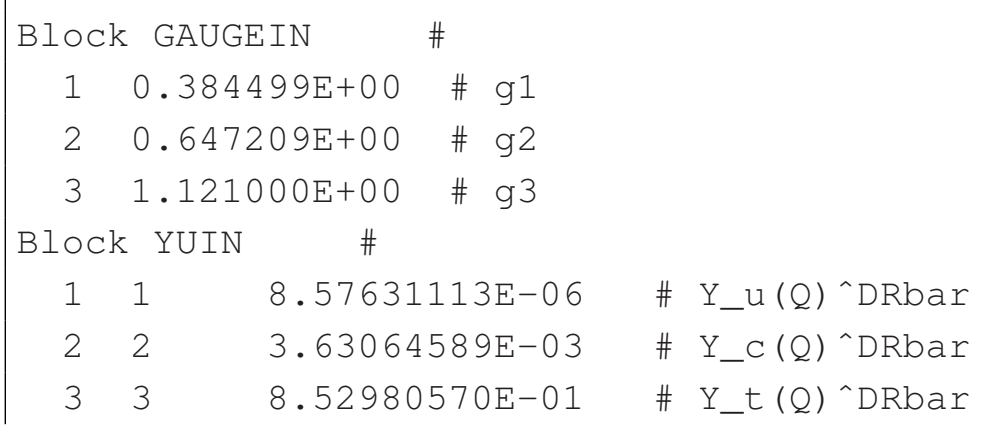

\footnotetext{
${ }^{1}$ We extend and partly depart from the SUSY LesHouches convention [61, 62] to obtain a greater flexibility for the implementation of new models.
} 
Calculation of decay widths The generated version of SPheno is capable of calculating the widths and branching ratios of all non-SM scalars and fermions. While only two body decays are taken in account for decaying scalars and vector bosons, the fermion widths also include the three body decays to all-fermion final states. In the case of Higgs fields, the decays into two-photon and two-gluon final states are included at leading order [63], as are the gluonic QCD corrections for the Higgs decays into quarks and squarks. [64, 65].

\subsection{Defining the properties of the generated SPheno version}

An additional file which defines the properties of the generated SPheno function is required by SARAH for generating SPheno output. This file must be located in the same directory as the other models files for SARAH. By default, it is assumed that this file is named SPheno.m, but it is also possible to use other file names (see sec. 3.6). This way it is easily possible to generate different SPheno implementations of the same model.

The content of the SPheno specific input file for SARAH is the following:

1. MINPAR: A list of parameters which should be read by SPheno from the block MINPAR in a LesHouches file. First, the number in the block is defined, afterwards the variable. For example:

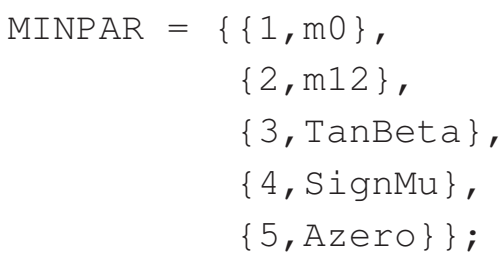

Later, the values of those parameters can be communicated to SPheno by using an input file which contains

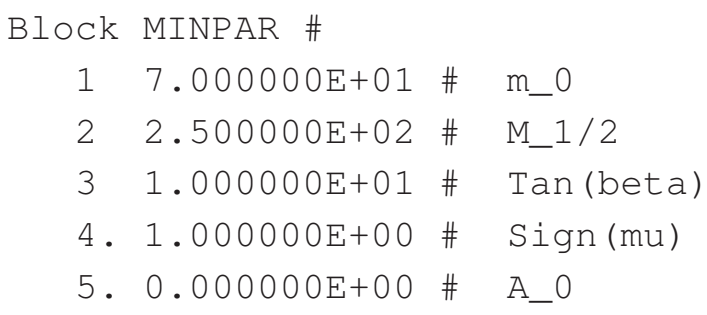

2. EXTPAR: It is also possible to define additional parameters for the block EXTPAR of the LesHouches input file by

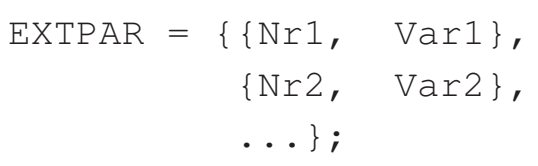

For instance, in order to give three additional VEVs as input, we can use 


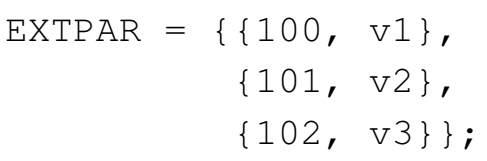

and set the values later on in the input file by

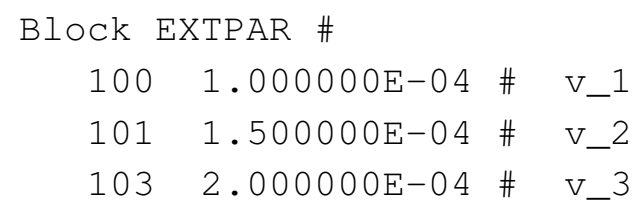

Note that there are no hard coded entries for MINPAR or EXTPAR. This makes it necessary to define these blocks also for models with already existing SLHA conventions. However, this also provides more freedom in varying the model and the free parameters.

3. RealParameters: By default, all parameters defined in MINPAR or EXTPAR assumed to be complex, i.e. it is possible to use also the block IMMINPAR to define the imaginary part. However, some Fortran functions like sin can't be used with complex numbers, therefore is is necessary to define parameters like $\tan \beta$ explicitly as real, e.g.

RealParameters $=\{$ TanBeta $\}$;

4. ParametersToSolveTadpoles: For each field which can obtain a VEV, SARAH derives the corresponding minimum condition. These equations constrain as many parameters as there are VEVs in the model. ParametersToSolveTadpoles defines which parameters the tadpole equations will be solved for.

For example, to use the standard choice in the MSSM $\mu, B_{\mu}$, the entry reads:

ParametersToSolveTadpoles $=\{\backslash[\mathrm{mu}], \mathrm{B}[\backslash \mathrm{mu}]\}$;

SARAH uses the Solve command of Mathematica to solve the tadpole equations for the given set of parameters. If the solution is not unique because a parameter $X$ appears squared, SARAH solves the equations for the absolute squared. The phase is then defined by the automatically generated variable SignumX, which is expected to be given as input. That's for instance the case of the $\mu$ parameter in the MSSM.

The solutions for the tadpole equations are applied by SPheno during the numerical analysis at the SUSY as well at the electroweak scale. For that purpose the running values of all parameters including the VEVs are taken as input at the considered scale.

5. UsegivenTapdoleSolution: In cases, in which Mathematica won't find an analytical solution for the tadpole equations for the given set of parameters, this 
variable has to be set to True and an approximated solution can be given. These solutions are defined by

- SubSolutionsTadpolestree: For the solution at tree level SubSolutionstadpolestree $=\{x 1->$ soll, $x 2->$ sol2,... $\}$;

Here, $x 1, x 2$ are the names of the parameters which are fixed by the tadpole equations and sol1, sol2 are the approximated expressions for them.

- SubSolutionsTadpolesLoop: The solutions of the one loop corrected tadpole equations. The one loop corrections to the different VEVs have to be named Tad1Loop [i] .

6. RenormalizationScaleFirstGuess: For the first run of the RGEs, before any mass has been calculated by SPheno, the squared renormalization scale can be defined by this entry. For example, for a mSugra scenario the common choice is

RenormalizationscaleFirstGuess $=\mathrm{mo}^{\wedge} 2+4 \mathrm{~m}^{2} 2^{\wedge} 2$;

This affects the running only if the SUSY scale is not fixed and SPA conventions are disabled in the LesHouches input file.

7. Renormalizationscale: For all further runs, another renormalization scale can be given which is a function of the calculated masses, e.g.

Renormalizationscale $=\operatorname{MSu}[1] * \operatorname{MSu}[6]$;

8. Two loop contributions to the Higgs masses: if the Higgs sector of the model is the same as for the MSSM, the original SPheno routines for calculating the two- loop tadpole equations and two-loop self energies to the the scalar and pseudo scalar Higgs can be activated by setting

UseHiggs2LoopMSSM = True;

9. Condition for the GUT scale: to set a condition for a dynamically adjusted GUT scale, use

ConditionguTscale = l.h.s == r.h.s;

A common choice would be the unification point of $g_{1}$ and $g_{2}$. In that case the condition reads

Conditiongutscale $=g 1==g 2$;

Note, that the value of the left-hand side must be smaller at scales below the GUT scale as the right hand side. 
10. Boundary Condition: It is possible to define boundary conditions at three different scales:

- Electroweak scale: BoundaryEWSBScale

- SUSY scale: BoundarysUSYScale

- GUT scale: BoundaryHighscale

In addition, if thresholds are involved, boundary conditions can be set at the threshold scale, see sec. 3.7. It is also possible to use a low scale input without any RGE running. In that case special boundary conditions can be defined by the array BoundaryLowscaleInput.

All boundaries are defined by a two dimensional array. The first entry is the name of the parameter, the second entry is the used condition at the considered scale. The condition can be...

- ... an input parameter from MINPAR or EXTPAR, e.g.

$\{$ MassB, m12\};

- ... a block in the SLHA input file, e.g.

$\{\mathrm{Yv}, \mathrm{LHInput}[\mathrm{YV}]\}$;

- ... a function of different parameters, e.g.

$\{\mathrm{TYd}, \mathrm{Azero}$ Y $\mathrm{d}\}$;

- ... a diagonal matrix, e.g.

$\left\{\mathrm{md} 2, \mathrm{DIAGONAL} \mathrm{mo}^{\wedge} 2\right\}$;

- ... matrix multiplications or the inverse of a matrix, e.g.

$\{\mathrm{X}, \operatorname{MatMul2}[\mathrm{A}$, InverseMatrix [B], Fortranfalse] ;

For the matrix multiplication MatMul2 has to be used. The third argument controls whether if only diagonal elements (Fortrantrue) should be considered or not ( FortranFalse).

- ... a self defined function

$\{X, \operatorname{Func}[A, B, C]\}$;

It is also possible to use some self defined function. The Fortran code of that function has to included in the array SelfDefinedFunctions in SPheno.m. Later on it will be written to Model_Data.f90. Note, that the standard functions needed for GMSB are already included [66]: 
- fGMSB $[\mathrm{X}]$ :

$$
\begin{aligned}
f(x)= & \frac{1+x}{x^{2}}\left(\ln (1+x)-2 \operatorname{Li}_{2}\left(\frac{x}{1+x}\right)+\frac{1}{2} \operatorname{Li}_{2}\left(2 \frac{x}{1+x}\right)\right)+ \\
& \frac{1-x}{x^{2}}\left(\ln (1-x)-2 \operatorname{Li}_{2}\left(\frac{x}{x-1}\right)+\frac{1}{2} \operatorname{Li}_{2}\left(2 \frac{x}{x-1}\right)\right)
\end{aligned}
$$

$-\operatorname{gGMSB}[\mathrm{X}]$ :

$$
g(x)=\frac{1+x}{x^{2}} \ln (1+x)+\frac{1-x}{x^{2}} \ln (1-x)
$$

Boundary conditions can be overwritten by assigning a value to a parameter in the LesHouches input file. For example, the Higgs soft breaking masses at the GUT scale can be forced to have specific values instead of $m_{0}^{2}$ by declaring

\begin{tabular}{|cll||}
\hline Block MSOFTIN & $\#$ & \\
21 & 10000.000 & $\#$ mHd2 \\
22 & 20000.00 & $\#$ mHu2 \\
\hline
\end{tabular}

in the SLHA file.

Several sets of boundary conditions In order to implement different versions of a single model which differ only by the used boundary conditions, BoundaryEWSBScale, BoundarySUSYScale, BoundaryHighScale can be also a nested list, e.g.

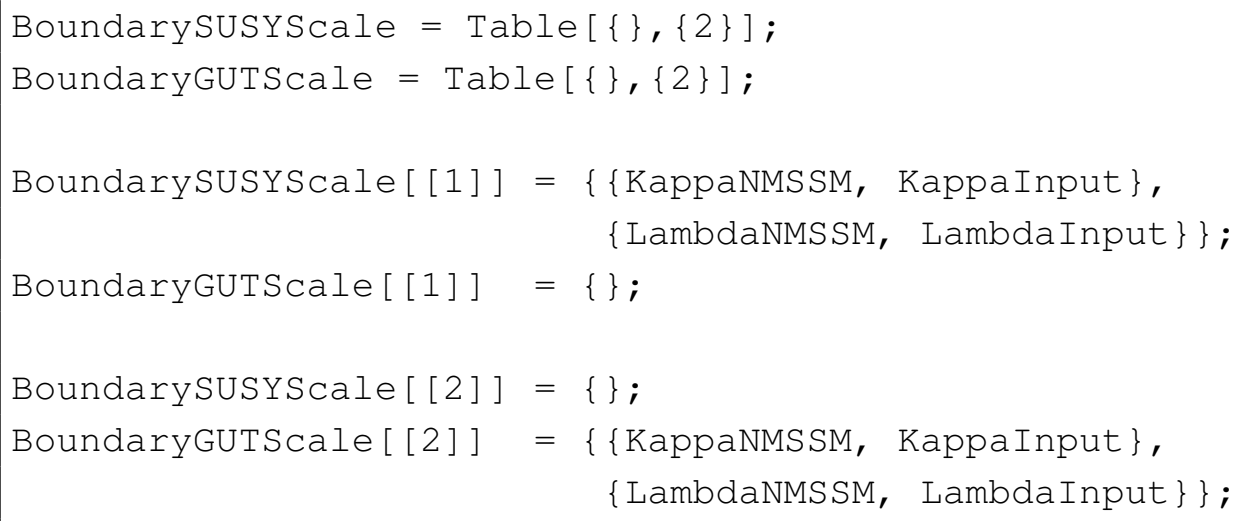

In the first case, the input values for $\lambda$ and $\kappa$ are taken at the SUSY scale, in the second one at the GUT scale. To communicate to SPheno which set of boundary conditions should be used for a run, flag 2 in MODSEL is used:

Block MODSEL \#

$2 \mathrm{X}$ \# This uses the $\mathrm{x}$. set of boundary conditions.

The default value is 1 .

11. Lists for calculating decay widths:

- ListDecayParticles: List of particles for which two-body decays will be calculated. This can be a list of particles using the names inside SARAH, e.g. 
ListDecayParticles $=\{\mathrm{Sd}, \mathrm{Su}, \mathrm{Se}, \mathrm{hh}, \mathrm{Ah}, \mathrm{Hpm}, \mathrm{Chi}\}$;

or just Automatic. If Automatic is used, the widths of all particles not defined as standard model particles as well as the top width are calculated by SPheno.

- ListDecayParticles3B; Three body decays of fermions. This can be a list with the names of the particles and the corresponding files names, e.g.

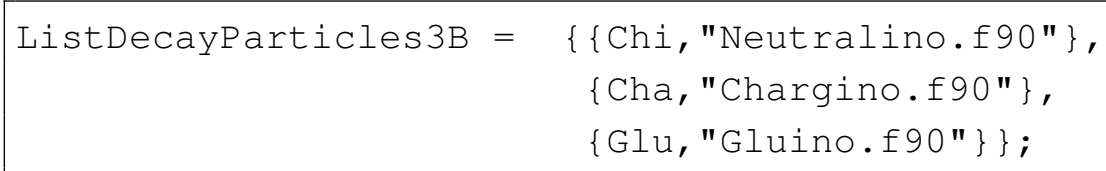

or just Automatic. If Automatic is used, the widths of all fermions not defined as standard model particles are calculated. The auto generated file names are ParticleName. 190.

12. Ordering of mass eigenstates: normally, all particles of one kind are ordered in SPheno by their mass. However, it might be desirable to override this behavior and instead define another ordering scheme. For example, consider several massless $\mathrm{CP}$ odd particles at tree level exist which can be assigned to a Goldstone boson. For this purpose, a condition can be defined by using

ConditionforMassordering $=\{$ Particle, Condition $\}, \ldots\}$;

The condition has to be Fortran source code and is added to the corresponding routine. For instance, a condition for the NMSSM would read

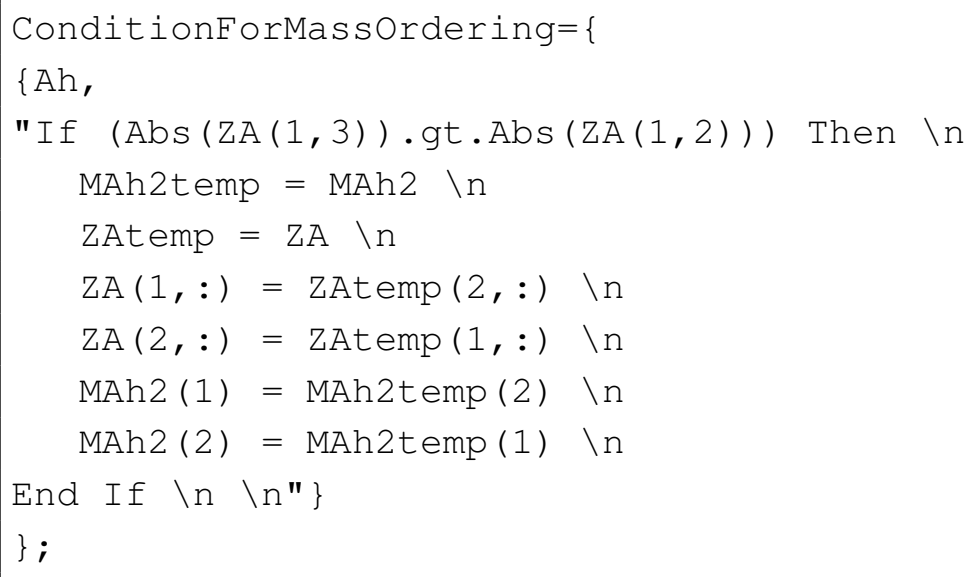

This example checks whether two massless pseudo scalars are present in the spectrum and, if this is the case, uses the not singlet-like particle as Goldstone boson.

\subsection{Example: The input file for the MSSM}

To generate the SPheno output for the MSSM, SPheno.m should include the following information: 
1. We want to have mSugra like boundary conditions. Therefore, we chose the minimal set of parameters defining the model as $m_{0}, M_{1 / 2}, A_{0}, \operatorname{sign} \mu$ and $\tan \beta$. These will later be read from the MINPAR block of a LesHouches input file.

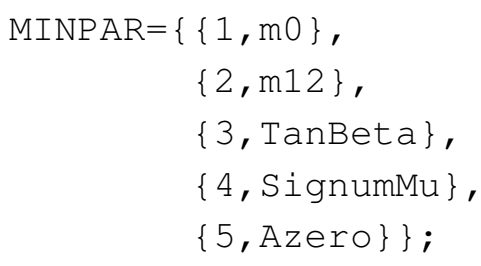

2. TanBeta has to be declared as a real parameter

RealParameters $=\{$ TanBeta $\}$;

3. As usual in the MSSM, the tadpole equations should be solved with respect to $\mu$ and $B_{\mu}$

ParametersToSolveTadpoles $=\{\backslash[\mathrm{Mu}], \mathrm{B}[\backslash[\mathrm{Mu}]]\}$;

4. To study models with a dynamically adjusted SUSY scale, the expressions for the definition of the SUSY scale can be given. The first expression is used only before the mass spectrum has be calculated the first time. Note, that these definitions can easily disabled in the LesHouches input file by flag MODSEL 12 and a fixed scale can be used. Also, when SPS conventions are switched on in the LesHouches input file by SPhenoInput 2, a fixed scale of $1 \mathrm{TeV}$ is used.

RenormalizationScaleFirstGuess $=\mathrm{m0}^{\wedge} 2+4 \mathrm{~m}^{\wedge} 2^{\wedge} 2$;

Renormalizationscale $=\operatorname{MSu}[1] \star \operatorname{MSu}[6]$;

5. The GUT scale is the unification point of $g_{1}$ and $g_{2}$

ConditionguTscale $=g 1==92$;

6. As said, we want to use mSugra like boundary conditions. These are straightforward define by

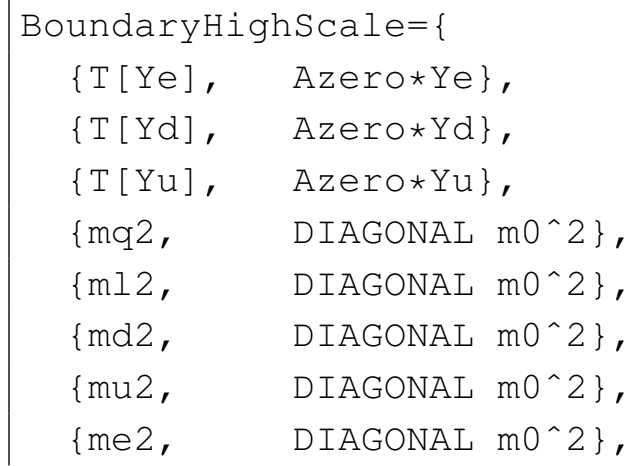




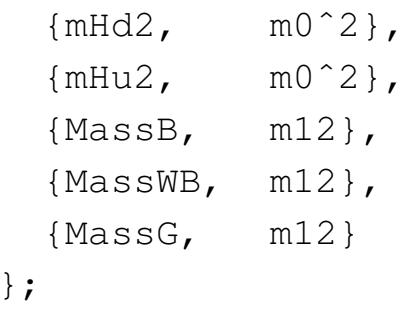

7. It is also possible to use the generated SPheno version with a low scale input. This is enabled by setting MODSEL 1 to 0 . In that case, input values for all free parameters of the model are expected. However, also in this case, we can define a set of boundary conditions, e.g. for dynamically calculating the SUSY VEVs

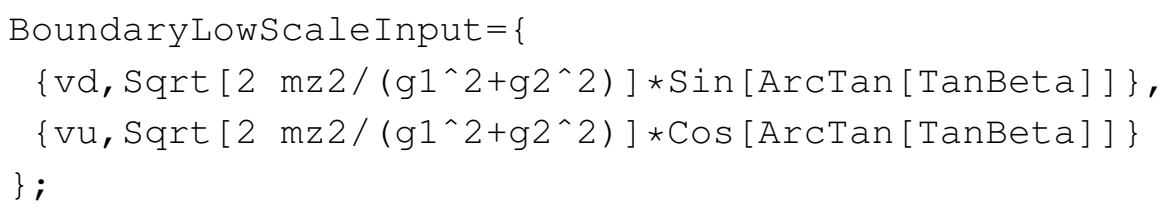

8. Finally, we define that the code for the calculation of the two and three body decays is generated for all SUSY particles. That's done by using the flag Automatic.

ListDecayparticles = Automatic;

ListDecayParticles3B = Automatic;

\subsection{Generating the output}

After an input file with all necessary information has been created, the generation of the source code for SPheno is triggered by

\section{In [5]: MakeSPheno [Options]}

The different options are:

- Eigenstates->Name of Eigenstates. If not specified, the last set of eigenstates is used which corresponds to the last entry of NameOfStates (see sec. 2.3.1).

- ReadLists->True can be used if all vertices and RGEs have already been calculated for the model and the former results should be used to save time.

- InputFile. The name of the SPheno input file. If not defined, SPheno.m is used.

The generated source code is located in

\section{[SARAH Directory]/Output/[Model]/[Eigenstates]/SPheno/}

It is sufficient to copy all files from this directory to a sub-directory NAME of SPheno version 3.1.4 or later and compile it using in the SPheno root directory

$>$ make Model $=\mathrm{NAME}$

NAME has to be the same as the name of the model defined in SARAH (see sec. 2.3.1). 


\subsection{Including Thresholds}

Using SARAH it is possible to include thresholds in the RGE running performed by SPheno.

\subsubsection{Thresholds without gauge symmetry breaking}

If all scales have the same gauge structure, it is possible for SARAH to derive the RGEs for all scales from the RGEs for the highest scale by performing the following steps:

- For those fields which should be integrated out during the run, variables $n_{g e n}\left(\Phi_{i}\right)$ are introduced internally which define the number of generation of the heavy field $\Phi_{i}$. All gauge group constants like the Dynkin index summed over chiral superfields, $S(R)$, are expressed as function of $n_{g e n}\left(\Phi_{i}\right)$. These $n_{g e n}\left(\Phi_{i}\right)$ are dynamically adjusted, when the energy scale crosses a threshold.

- When crossing a threshold, the couplings involving heavy fields are set to zero. For example, the Yukawa type coupling of the form $Y^{i j} \Phi_{i} \phi_{j} H$ involves three generations of the heavy field $\Phi$. At the threshold of $\Phi_{k}$, the $k$-th row of $Y$ is set to zero. That happens similarly for all other superpotential and soft-breaking parameters.

- The masses of scalar and fermionic components of the heavy superfields are assumed to be identical, i.e. the soft SUSY breaking terms are assumed to be negligible. These masses are given by a bilinear superpotential term.

In order to include thresholds without gauge symmetry breaking, the following steps have to be performed:

1. The heavy fields must be marked for deletion in the SARAH model definition:

Deletefields $=\{\ldots\}$;

This ensures, that the decays, loop corrections, etc. at the SUSY scale calculated by SPheno receive no contributions from the heavy fields

2. The thresholds have to be defined in SPheno.m :

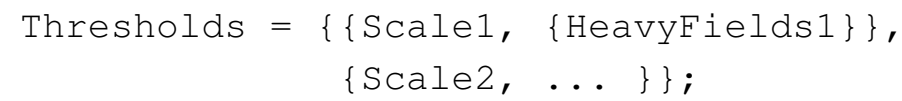

For all scales an entry in the array Thresholds has to be added. Each entry defines the threshold scale and at second position a list of the heavy superfields which can be restricted to specific generations.

It is possible to define boundary conditions at each threshold scale for running up and down separately:

BoundaryConditionsUp $[\mathrm{x}]]=\{\ldots\}$;

BoundaryConditionsDown $[\mathrm{x}]]=\{\ldots\}$; 
Threshold corrections Using 2-loop RGEs requires 1-loop boundary condition. Therefore, at each threshold scale the one loop threshold corrections to gauge couplings and gaugino masses are calculated. The general expressions are [14]

$$
\begin{aligned}
g_{i} & \rightarrow g_{i}\left(1 \pm \frac{1}{16 \pi^{2}} g_{i}^{2} I_{2}^{i}(r) \ln \left(\frac{M^{2}}{M_{T}^{2}}\right)\right), \\
M_{i} & \rightarrow M_{i}\left(1 \pm \frac{1}{16 \pi^{2}} g_{i}^{2} I_{2}^{i}(r) \ln \left(\frac{M^{2}}{M_{T}^{2}}\right)\right) .
\end{aligned}
$$

$I_{2}^{i}(r)$ is the Dynkin index of a field transforming as representation $r$ with respect to the gauge group belonging to the gauge coupling $g_{i}, M$ is the mass of this particle and $M_{T}$ is the threshold scale.

Example As an example, a version of SPheno implementing the seesaw type II and type III models can be generated by adding the following entries to Spheno.m

1. Seesaw II:

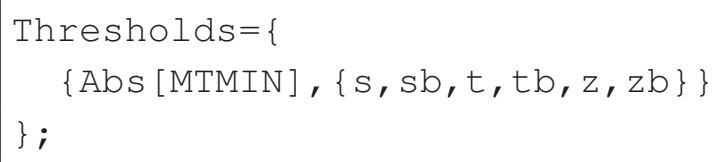

2. Seesaw III:

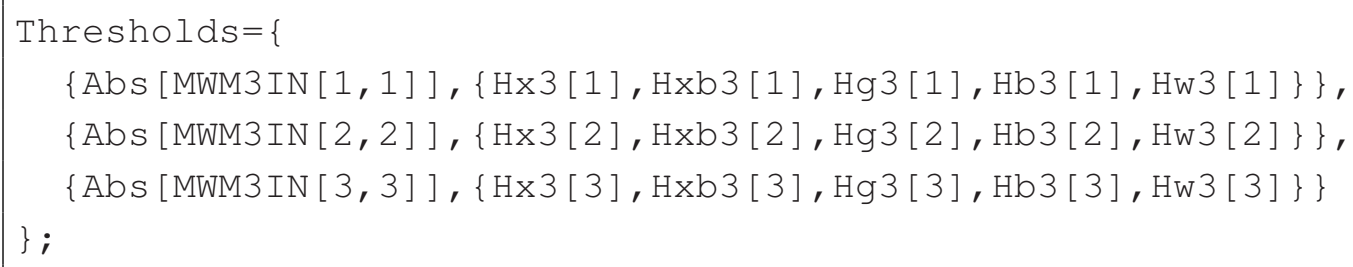

For a more comprehensive discussion of the model files for the Seesaw I, see appendix A.1.

\subsubsection{With gauge symmetry breaking}

If the gauge structure at the different scales are different, each set of RGEs is calculated separately and this information is then combined into one consistent version of SPheno which includes routines for calculating finite shifts in the gauge couplings, gaugino and scalar mass parameters. As an example, the implementation of a left-right supersymmetric model with one symmetry breaking scales is shown in sec. A.2.

In order to implement such a model, the following steps are necessary:

1. For each regime, a separate model file for SARAH has to be created. These model file have to be saved in the subdirectories Regime-1, Regime-2, ... of

[SARAH Directory]/Models/[Model]/

beginning with the highest scale. 
2. The SPheno input file for the higher scales must provide the following information:

- Intermediatescale = True

- Regimenr $=\mathrm{X}$

- A list of the heavy fields, which should be integrated out, the gauge sector below the threshold as well as the corresponding quantum numbers of the fields which are to be integrated out. This is needed to calculate the finite shifts at the threshold scale, for instance

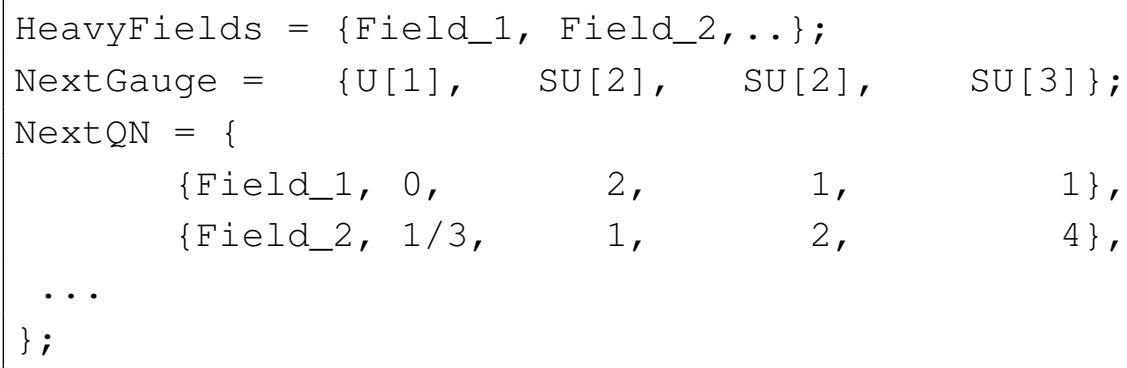

Care has to be taken to use the same ordering of the $S U(2)$ gauge groups as in the orignal definition.

3. All necessary information for combining the regimes to one SPheno is given in SPheno.m of the lowest scale.

- IntermediateScale = False

- Regimenr = X

- The threshold scales: ThresholdScales $=\ldots$

- The boundary conditions for running up and down at each threshold scale:

BoundaryConditionsUp $[\mathrm{x}]]=\{\ldots\}$;

BoundaryConditionsDown $[\mathrm{x}]]=\{\ldots\}$;

In the boundary conditions index 1 , index $2, \ldots$ can be used for defining sums over indices.

- The usual information for SPheno, defined in the sec. 3.4.

When starting the SPheno output of the lowest scale, all other scaler are evolved automatically. Note that, in order to calculate the RGEs of the different regimes, SARAH starts one additional Mathematica kernel. For passing the information between the different Mathematica kernels a directory Meta in the model directory is created by SARAH. The screen output of Mathematica during the evaluation of the higher regimes is written to that directory (Output-Regime-X.m), allowing the user to supervise the progress and see potential error messages. The necessary information of each regime for writing the combined source code for SPheno at the end is saved by SARAH in the files Regime-X.m. 


\subsection{Low energy SPheno version}

It is also possible to create a SPheno version with much less features which only accepts low energy input. That means, the RGEs are not written out and also the fit to the electroweak data is not performed in the numerical evaluation of one point. It just solves the tadpole equations, calculates the tree- and one-loop masses as well as the decay widths and branching ratios. The advantage of such a SPheno version is that it works with a larger set of models, e.g. also non-SUSY models or other models not supported by a full evaluation as explained in sec. 3.10. To get a SPheno version without RGE evolution, insert

OnlyLowEnergySPheno = True;

in SPheno.m. The remaining information needed by SARAH is only a small subset of the settings discussed above and consists of

- MINPAR

- ParametersToSolveTadpoles

- BoundaryLowScaleInput

- ListDecayParticles and ListDecayParticles3B. Note that the Automatic statement for automatically deriving the decays of all non-SM particles does not work in this case as SARAH doesn't differ between SUSY or Non-SUSY particle in order to make the output as generic as possible. Therefore, the lists of the decaying particles have to be supplied manually.

\subsection{Differences to SPheno3.1.4}

A few things are handled in a slightly different way in a SPheno MSSM module created by SARAH in comparison to SPheno 3.1.4. Also, some things are not yet implemented. We give an overview in Tab. 1.

\subsection{Supported models and known issues}

While SARAH can create valid SPheno code for many different models, there are some requirements on the model and some minor restrictions on the functionality of the resulting SPheno module. At the moment, those are

- Fit to low energy data: in order to perform a fit to low energy data (e.g. for fermion masses, $m_{Z}, G_{F}$ and $\alpha_{e m}$ ) as starting point of the RGE evaluation, the following parameters must be present in the model: Yukawa couplings for lepton and quarks, two Higgs VEVs and, of course, the three SM gauge couplings and the SM particle content. However, it is still possible to use at least some features of the SPheno output of SARAH by manually supplying model parameters for SPheno. In that way, the RGE evaluation and the fit the electroweak data is skipped, but the one-loop corrected masses as well as the decay widths and branching ratios are calculated. 


\begin{tabular}{|c|c|c|}
\hline & SPheno3.1.4 & SPheno by SARAH \\
\hline $\begin{array}{l}\text { Loop corrections in } \\
\text { Higgs sector }\end{array}$ & $\begin{array}{l}\text { Complete one-loop correc- } \\
\text { tions and dominant two-loop } \\
\text { corrections. }\end{array}$ & $\begin{array}{l}\text { One-loop corrections are cal- } \\
\text { culated by SARAH. Dominant } \\
\text { two-loop corrections imple- } \\
\text { mented in SPheno can be } \\
\text { linked. }\end{array}$ \\
\hline Tadpole equations & $\begin{array}{l}\text { Solved at SUSY scale. Solu- } \\
\text { tions are evaluated at the EW } \\
\text { due to RGE running }\end{array}$ & $\begin{array}{l}\text { Solved at SUSY and EW } \\
\text { scale. }\end{array}$ \\
\hline Three body decays & $\begin{array}{l}\text { Three body decays of fermions } \\
\text { and stop }\end{array}$ & Three body decays of fermions \\
\hline $\begin{array}{l}\text { Loop induced } \\
\text { neutralino decays }\end{array}$ & $\begin{array}{l}\text { One-loop decay into photon } \\
\text { and neutralino included }\end{array}$ & $\begin{array}{l}\text { No loop induced decays in- } \\
\text { cluded }\end{array}$ \\
\hline $\begin{array}{l}\text { Loop induced } \\
\text { gluino decays }\end{array}$ & $\begin{array}{l}\text { One-loop decay into gluon } \\
\text { and neutralino included }\end{array}$ & $\begin{array}{l}\text { No loop induced decays in- } \\
\text { cluded }\end{array}$ \\
\hline$e^{+} e^{-}$collisions & Calculates cross sections & $\begin{array}{l}\text { Cross section calculation not } \\
\text { included }\end{array}$ \\
\hline
\end{tabular}

Table 1: Different handling and implementation status in SPheno 3.1.4 vs. the SPheno modules created by SARAH.

- Flavor decomposition: with SARAH it is possible to assign a unique name to each generation of a particular field and this way treat the individual generations as independent fields. That is not yet supported in the SPheno output. Furthermore, mixing matrices generated with the option NoFlavorMixing can not yet be handled by the numerical code.

\section{Checking Higgs constraints with HiggsBounds}

HiggsBounds [47, 48] is a tool to test the neutral and charged Higgs sectors against the current exclusion bounds from the Higgs searches at the LEP, Tevatron and LHC experiments. The required input consists of the masses, width and branching ratios of the Higgs fields. In addition, it is either possible to provide full information about production cross sections in $e^{+} e^{-}$and $p p$ collisions, or to work with a set of effective couplings. HiggsBounds can be downloaded from

http://projects.hepforge.org/higgsbounds

Although HiggsBounds supports the LesHouches interface, this functionality is restricted so far to at most 5 neutral Higgs fields, and therefore, we don't use it. Instead, SPheno modules generated by SARAH can create all necessary input files needed for a run of HiggsBounds with effective couplings (option whichinput=effC). To write these 
files, the flag 75 in the block SPhenoInput in the LesHouches input file has to be set to 1 .

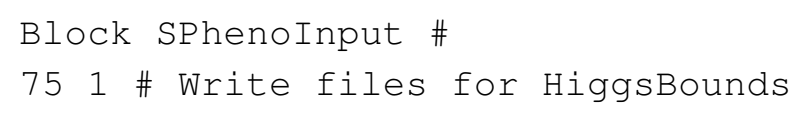

Unfortunately, we can not provide all information which can be used by HiggsBounds to check the constraints. In particular, the effective couplings $H \rightarrow \gamma Z$ and $H \rightarrow g g Z$ are not yet calculated by SPheno and therefore set to zero in the output. In addition, as already mentioned, the SPheno version created by SARAH does not support calculating the $e^{+} e^{-}$ cross sections. For this reason, also the LEP production cross section of charged Higgs fields is not available for SPheno and it sets this value also to 0. However, it is of course possible to calculate this cross section as well as all other cross sections needed for the options (whichinput=hadr or whichinput=part) of HiggsBounds using CalcHep or WHIZARD with the corresponding model files created by SARAH (cf. sec. 5 and 6).

The following files are written by SPheno

- MH_GammaTot.dat:

Masses and widths of all neutral Higgs fields

- MHplus_GammaTot.dat:

Masses and widths of all charged Higgs fields

- BR_H_NP . dat:

Branching ratios of neutral Higgs fields into invisible and other neutral Higgs fields.

- BR_Hplus.dat:

Branching ratios of charged Higgs fields into $c \bar{s}, c \bar{b}$ and $\tau \bar{\nu}$ final states

- BR_t.dat:

Top quark branching rations into a bottom quark plus either a $W$ boson or a charged Higgs

- effC.dat: Effective couplings among neutral Higgs fields and of neutral Higgses to $s \bar{s}, c \bar{c}, b \bar{b}, t \bar{t}, \mu \bar{\mu}, \tau \bar{\tau}, \gamma \gamma, g g, \gamma Z, g g Z$

- LEP_HPHm_CS_ratios.dat:

LEP cross sections for the production of charged Higgs bosons (set to zero, see above)

HiggsBounds can be run on the generated files by invoking it as

$>$./HiggsBounds LandH effC [NN] [NC] [SPheno Directory]

where [NN] has to be replaced by the number of neutral Higgses and [NC] by the number of the charged ones. Please consult the HiggsBounds manual for further information. The results of the check are written to the file HiggsBounds_results. dat which is located in the same directory as the input file, i.e. in our case in the SPheno root directory. 


\section{Calculation of cross sections, widths and relic density using $\mathrm{Ca}$ lcHep and micrOMEGAs}

CalcHep $[45,44]$ is a package for the calculation of Feynman amplitudes and their integration over multi-particle phase space. It is designed to provide a direct transition from the Lagrangian to the cross sections and distributions. The CalcHep homepage is located at

http://theory.sinp.msu.ru/ pukhov/calchep.html

\subsection{Using CalcHep with SARAH and SPheno}

The CalcHep model files produced by SARAH support both the Feynman and unitarity gauges. Furthermore, SARAH can split interactions between four colored particles as required by CalcHep/CompHep. Models with CP violation are also supported. The model files for $\mathrm{CalcHep/CompHep} \mathrm{are} \mathrm{created} \mathrm{by}$

In [5]: MakeCHep [Options];

Options exist for specifying the gauge (FeynmanGauge $\rightarrow$ True/False) and for activating the support for $\mathrm{CP}$ violation (CPViolation $\rightarrow$ True). In addition, the splitting of specific four-scalar interactions can be suppressed as long as they are not colored (NoSplitting $\rightarrow$ list of fields) and the running of the strong coupling constant can be included as it is usually done in the standard CalcHep files (UseRunningCoupling $\rightarrow$ True).

Recently, it became also possible to use SLHA files with CalcHep to provide the numerical values of the parameters [67]. SARAH uses this option if the flag SLHAinput $\rightarrow$ True is used. Thus, the parameters calculated by SPheno can be directly passed to CalcHep.

In order to calculate the same process for different points in parameter space, the so called blind mode of $\mathrm{CalcHep}$ can be used for performing calculations without the need to start the graphical interface. The blind mode is most conveniently put to work using the following steps:

1. Start CalcHep, insert a process and choose make $n_{-}$calchep. This creates an executable file called $\mathrm{n}_{-} \mathrm{calchep}$ in the results subdirectory of your CalcHep model.

2. In order to keep a copy of the files, copy the content of results to another directory (make sure that the subdirectory aux is included in the copy). The spectrum file generated by SPheno goes into the same directory.

3. Change to the directory containing $n_{-} c a l c h e p$ and start it via

$$
>\text {./n_calchep tolind }
$$

4. Make the desired adjustments and start the numerical integration using Vegas. 
5. When $n_{-}$calchep exits, a line of the form [ \{]$][\{\{[]]$is returned which mimics the keyboard commands you did

6. Now, the same calculation can be repeated without the CalcHep frontend using the command

$>$./n_calchep -blind " [ \{]$][\{\{[]] "$

This way, a number n_calchep instances for several processes (e.g. sparticle or Higgs production at LEP, Tevatron or LHC) can be created and called non-interactively from a script, thus facilitating automatized scans over parameter space.

\subsection{Relic density calculations with micrOMEGAs}

micrOMEGAs [46] is a well known tool for the calculation of the relic density of a dark matter candidate. The download is located at

http://lapth.in2p3.fr/micromegas/

As micromegAs uses CalcHep for the calculation of (co-)annihilation cross sections, a model file for CalcHep must be generated first before micrOMEGAs can be used.

SARAH writes two files for micrOMEGAs which can serve as so-called main files, i.e. they can be compiled with micrOMEGAs and executed to perform calculations. While Calcomega.cpp calculates only $\Omega h^{2}$ and writes the result to the file omg.out. Calcomega_with_DDetection.cpp computes also direct detection signals. As the SLHA+ import functionality of CalcHep can also be used with micrOMEGAs, it is sufficient to simply copy the spectrum file written by SPheno to the directory of micrOMEGAs and start the calculation.

\section{Monte Carlo studies with WHIZARD}

\subsection{Introduction}

WHIZARD [50] is a fast tree-level Monte Carlo generator for parton level events. A particular strength of the code is the efficient generation of unweighted events for high multiplicity final states (simulations with 8 final state particles have been performed successfully) using exact matrix elements. This makes it particularly useful for the study of supersymmetric models which generically feature complicated multiparticle final states arising from long decay chains.

Behind the scenes, WHIZARD builds on the optimizing matrix element generator $O^{\prime}$ Mega [49]. In order to deliver the fast tree level matrix elements required by WHIZARD, $\mathrm{O}^{\prime}$ Mega builds up matrix elements as directed acyclical graphs of one-particle off shell wave functions (1POW, Green's functions with all but one legs amputated). These graphs are then transformed into highly optimized FORTRAN 90 code which is called by WHIZARD to calculate helicity matrix elements by recursively fusing 1POWs. This algorithm is guaranteed to avoid any redundancies arising from the repeated evaluation of subdiagrams from the start and can be shown to grow only exponentially in complexity with the number of 
external legs (as opposed of the factorial growth of any traditional Feynman diagram based approach).

For the treatment of color, WHIZARD and $\mathrm{O}^{\prime}$ Mega leverage the color flow decomposition [68]. The amplitudes are decomposed into all possible different color flows, for which the amplitudes are then calculated using $\bigcirc^{\prime}$ Megas recursive algorithm and finally combined into the squared and color summed matrix elements. In addition to the fast calculation of the color trace, this algorithm also provides the color connection information later required by fragmentation and hadronization algorithms.

The integration and event generation in WHIZARD is performed using the adaptive multichannel Monte Carlo code VAMP [69]. For each process, WHIZARD dynamically determines a set of suitable phase space maps. VAMP assigns a grid to each map, all which are then linearly combined to form the phase space parameterization. During the integration process, VAMP adapts both the grids and the weights of the different channels. After integration, the optimized grids are used to facilitate the efficient generation of unweighted events.

Recently, WHIZARD has received a major upgrade, going from the (now legacy) 1.x version branch (currently 1.97) to the modern 2.x branch (currently 2.0.5). While the interface between SARAH and WHIZARD supports both branches, the new version features both many new physics features (including factorized matrix elements incorporating full spin and color correlations) and technical improvements, so using the newer version is highly advised. The WHIZARD package can be downloaded from

http://www.hepforge.org/archive/whizard/

\subsection{The interface between SARAH and WHIZARD}

The interface between SARAH and WHIZARD shares significant parts of its code with the interface between FeynRules [70], with a thin layer on top to interface with SARAH. As such, the feature set is identical with that of the FeynRules interface and, although we will now give a short overview, we point the reader to above reference for more information.

At the moment, the interface can handle spin $0, \frac{1}{2}$ and 1 fields, with support for spin $\frac{3}{2}$ being being planned for a future revision. The supported interactions are a subset of the operators currently available in $\mathrm{O}^{\prime}$ Mega. Specifically, up to very few exceptions, nearly all dimension 3 and 4 interactions are available, together with a couple of higher dimension operators. In particular, this list is sufficient to handle all interactions which are generated in a typical application of SARAH to a supersymmetric model without higher-dimensional terms in the Lagrangian.

The list of supported color structures is more complicated, but in practice all color tensors arising from QCD gauge interactions which lead to supported Lorentz structures are supported. Again, this covers all interactions which can be generated by SARAH. A full list of the supported color structures can be found in [71].

The 2.x branch of WHIZARD supports running the strong coupling, and the interface supports this functionality. In order to use it, the model must define a parameter called aS which is treated by the interface as $\alpha_{S}$ at the $Z$ pole. In addition, the QCD coupling 


\begin{tabular}{|l|l|}
\hline WOWhizardVersion & WHIZARD versions supported \\
\hline \hline "1.92" & 1.92 \\
\hline "1.93" & $1.93-1.95$ \\
\hline "1.96" & $1.96+$ \\
\hline "2.0" & $2.0-2.0 .2$ \\
\hline "2.0.3" (default) & $2.0 .3+$ \\
\hline
\end{tabular}

Table 2: Currently available version choices when generating WHIZARD model files, together with the respective WHIZARD versions supported by them.

constant $g_{S}$ must be derived from $\alpha_{S}$ and have the description "Strong-Coupling". Once these criteria are met, the generated model will automatically evolve all vertex factors which depend on either $g_{S}$ or $\alpha_{S}$ if the running coupling is activated in WHIZARD. For example, all model files included in SARAH support the running by deriving all vertices as function of $g_{3}$ and including the following definitions into the parameter list:

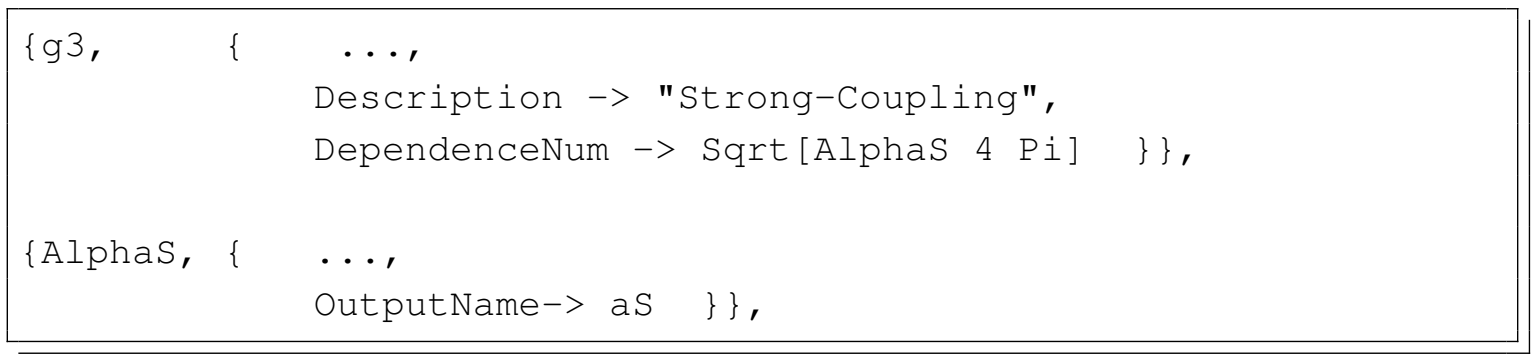

\subsection{Generating model files and using them with WHIZARD}

\section{Calling the interface}

In order to generate model files for WHIZARD, the Modeloutput command is used

In [3]: Modeloutput [Eigenstates, WriteWHIZARD->True];

This writes the model file using the default options. An equivalent syntax which allows for passing options to the interface is

In [3]: ModelOutput [Eigenstates];

In [4]: MakeWHIZARD [Options];

The different options accepted by MakeWHIZARD are:

1. Exclude, Values: list of generic vertex types, Default: \{SSSS \}

Prevents vertices matching the generic types from being generated in order to speed up the program and reduce the complexity of the generated model.

2. WOModelName, Values: string, Default: predefined model name

Gives the possibility to change the model name. If output for WHIZARD 1.x is generated, the name should start with $f r_{-}$in order for the model to be picked up automatically by the WHIZARD build system. 
3. MaximalCouplingsPerFile, Values: Number, Default: 500

Defines the maximal number of couplings written to one file. Adjusting this eases the workload on the compiler when compiling the model.

4. Version, Values: String, Default: latest version

Defines the version of WHIZARD for which the model file is generated. A list of all values for this setting currently valid can be found in Tab.2. In addition, you can get a list via ?WO 'WhizardVersion in Mathematica after SARAH has been loaded.

5. ReadLists, Values: True or False, Default: False

This setting controls whether the cached results from a previous calculation should be used.

While generating the model files, the interface will print status messages and information about potential incompatibilities of the model with WHIZARD to the screen. It is highly advised to read through this information carefully.

\section{Using the generated model files with WHIZARD}

After the interface has completed, the generated files can be found in the WHIZARD_Omega subdirectory of SARAH's output directory. In order to use the model with WHIZARD 2.x, the generated code must be compiled and installed. For most applications, this is done by simply issuing (inside the output directory)

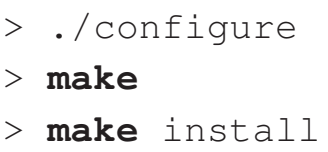

By default, the third command installs the compiled model into . whi zard in current user's home directory where it is automatically picked up by WHIZARD. Alternative installation paths can be specified using the --prefix option to WHIZARD.

>./configure --prefix=/path/to/installation/prefix

If the files are installed into the WHIZARD installation prefix, the program will also pick them up automatically, while WHIZARD's --localprefix option must be used to communicate any other choice to WHIZARD. In case WHIZARD is not available in the binary search path, the WO_CONFIG environment variable can be used to point configure to the binaries

>./configure WO_CONFIG=/path/to/whizard/binaries

More information on the available options and their syntax can be obtained with the -- help option.

In the case of WHIZARD 1.x output, the generated files must be patched into the WHIZARD source tree. To this end, the interface creates a script called inject. In most cases, it is sufficient to simply call the script as

$>$./inject /path/to/whizard

(from within the output directory). Issuing . / inject --help will display a list of options which can be used to adapt the script to more complicated usage scenarios. 


\section{Spectra and parameters}

In order to communicate the numerical values of the parameters calculated by SPheno to WHIZARD, each SPheno version generated by SARAH is capable of writing out a separate file providing this information as SINDARIN code which can be directly included into the WHIZARD input script. The user just has to make sure to use

Block SPhenoInput \#

761 \# WHIZARD file

in the LesHouches input file of SPheno. The created file WHIZARD.par. [Model] can be included in the input script for WHIZARD by means of the include statement. For example, for the MSSM, the corresponding line would read

include ("WHIZARD.par.MSSM")

\section{Parameter scans with SSP}

In the previous sections, we have discussed the implementation of SUSY models into different tools which can be combined to cover much of the analysis of new models. The corresponding work flow is depicted in Fig. 2.

\begin{tabular}{|c|}
\hline Define Model in SARAH \\
\hline$\downarrow$ \\
\hline $\begin{array}{c}\text { Derive necessary information: couplings, masses, loor } \\
\text { contributions, RGEs, decay channels, ... }\end{array}$ \\
\hline$\downarrow$ \\
\hline Generate model dependent source code for SPheno \\
\hline$\downarrow$ \\
\hline Compile and run SPheno \\
\hline$\downarrow$ \\
\hline $\begin{array}{c}\text { Give masses/parameters to WHIZARD, CalcHep, } \\
\text { micrOMEGAs and HiggsBounds }\end{array}$ \\
\hline
\end{tabular}

Figure 2: The model is defined in SARAH. Afterwards, SARAH generates all necessary files to implement this model in SPheno, micrOMEGAs and WHIZARD. In addition, the SPheno version writes a file which can be used as input for HiggsBounds. This provides an completely automatized way from model building to phenomenology.

However, in order to use this tool chain it is still necessary to run all programs and pass the information between them. To ease this task, we have created the SSP (SARAH Scan and Plot) Mathematica package. This tool facilitates a quick overview of a new model by performing the different analysis steps automatically for many different points in parameter space. Furthermore, it is possible to define additional parameter space constraints and to use Mathematica's intrinsic functions for creating 2D parameter space plots. 


\subsection{Installation and running scans}

The package can be downloaded from

http://projects.hepforge.org/sarah/ssP.html

After extracting the package archive to the Mathematica application directory, SSP is loaded via

In [1]: $<<$ "SSP/SSP.m"

A scan is started by

In [2]: Start ["Inputfile"];

where Inputfile is a file containing all necessary information for a scan.

\subsection{The input files}

Each scan performed by SSP is based on the information given in two input files. In a first file, which has to be located in the root directory of SSP, the location of the different programs is given and some basic information about the different tools like the name of the in- and output file has to be defined. For instance, the necessary entries for SPheno read

DEFAULT [SPheno $]=$ "[SPheno Directory]/bin/SPheno[Model]";

DEFAULT [SPhenoInputFile] = "LesHouches.in.[Model]";

DEFAULT [SPhenoSpectrumFile] = "SPheno.spc.[Model]";

The second file contains all information defining a specific scan: the name of the requested settings file, the programs which should be included into the scan, input parameters and ranges, any constraints which should be applied and the definitions of the actual plots. As several different scans can be defined in one input file, each scan has first to be assigned an unique identifier, e.g.

RunScans $=\{\mathrm{ScanM0}, \mathrm{ScanM12}\}$;

In order to toggle the inclusion of the different tools, the flags IncludeWHIZARD, IncludeHiggsBounds, IncludeCalcHep or IncludeMicrOmegas are used

DEFINITION [ScanM0] [IncludeHiggsBounds] = True;

DEFINITION [ScanM12] [IncludeHiggsBounds] = False;

The information on the parameters is defined by different lists for each block of the LesHouches input file which might look like

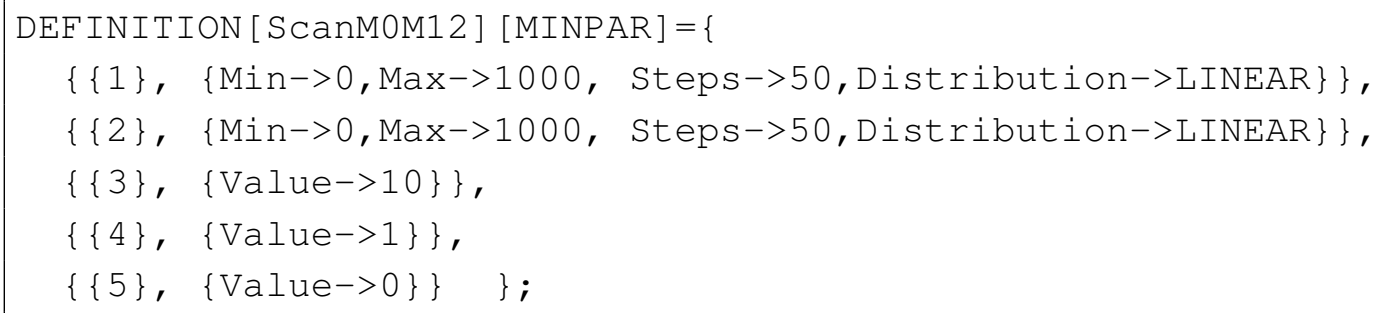


Other possible distributions are LOG and RANDOM.

\subsection{Features}

\section{Fit to constraints}

SSP can be instructed to adjust one or several model parameters such that a list of constraints is fulfilled. To this end, the Mathematica function NMinimize is utilized to find a combination of free parameters which leads to the smallest $\chi^{2}$ for the given constraints. For instance, to fix the Higgs mass between 114.9 and $115.1 \mathrm{GeV}$ by a variation of $A_{0}$ and $\tan \beta$, the necessary input is

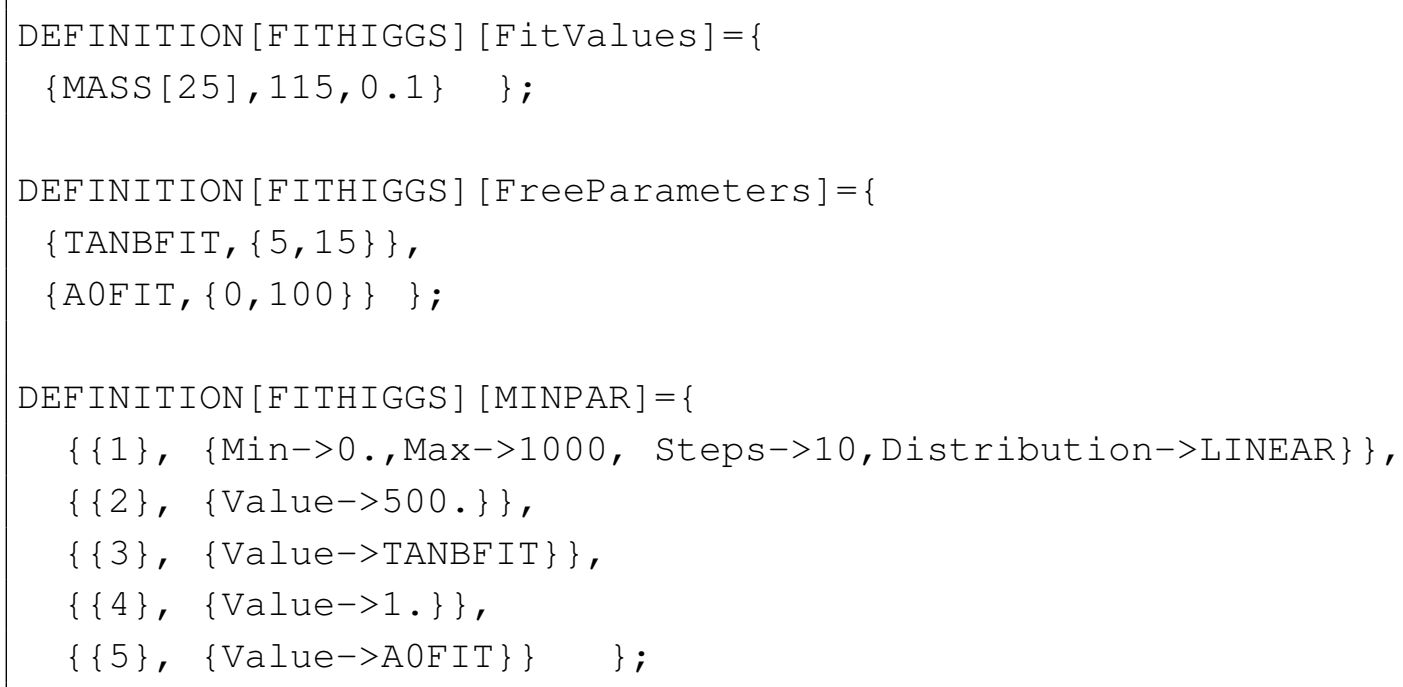

In order to tune the behavior of NMinimize, e.g. manually choosing a fit algorithm, options can be passed to the function

DEFINITION[FITHIGGS] [Fitoptions] =\{Method->"NelderMead" $\}$;

As most of the fit algorithms cannot deal with a finite parameter range $[a, b]$, SSP transforms to infinite boundaries via stretching

$$
P_{e x t}=a+\frac{b-a}{2}+\frac{\operatorname{atan}\left(P_{i n t}\right)}{\pi}(b-a)
$$

with $P_{\text {int }} \in[-\infty,+\infty]$ and $P_{\text {ext }} \in[a, b]$. For more details on NMinimize and its various options, we refer to the Mathematica manual.

\section{D parameter sampling}

One common problem is the sampling of a two dimensional parameter space, e.g. when checking the dark matter relic density in the $\left(m_{0}, M_{1 / 2}\right)$ plane. However, in many cases, a fixed grid or random scan might not be the best choice because certain areas should be sampled more precisely than others. The ContourPlot function of Mathematica was developed exactly for such purposes and therefore, SSP can be instructed to use it.

The input for using ContourPlot with SSP to do a $\left(m_{0}, M_{1 / 2}\right)$ scan is 


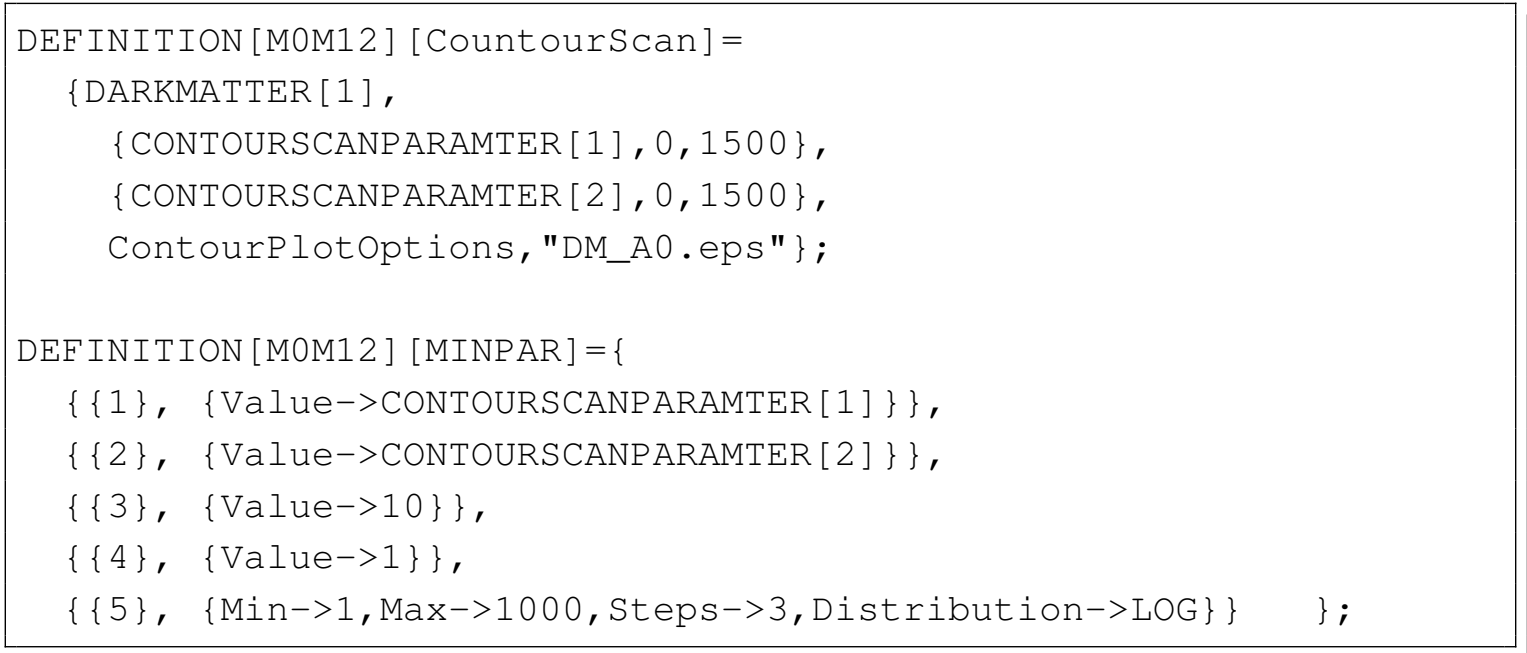

With this setup, contour plots for the relic density in the $\left(m_{0}, M_{1 / 2}\right)$ plane are created for $A_{0}=1,10,1000 \mathrm{GeV}$ and $\tan \beta=10, \operatorname{sign} \mu>0$. As options for the scan, the usual options of ContourPlot can be used. For instance, in Fig. 3, we used PrecisionGoal-> "Quality" and varied the PlotPoints between 5 and 50 . The number of valid parameter points which have been evaluated can be found in Tab. 3 .
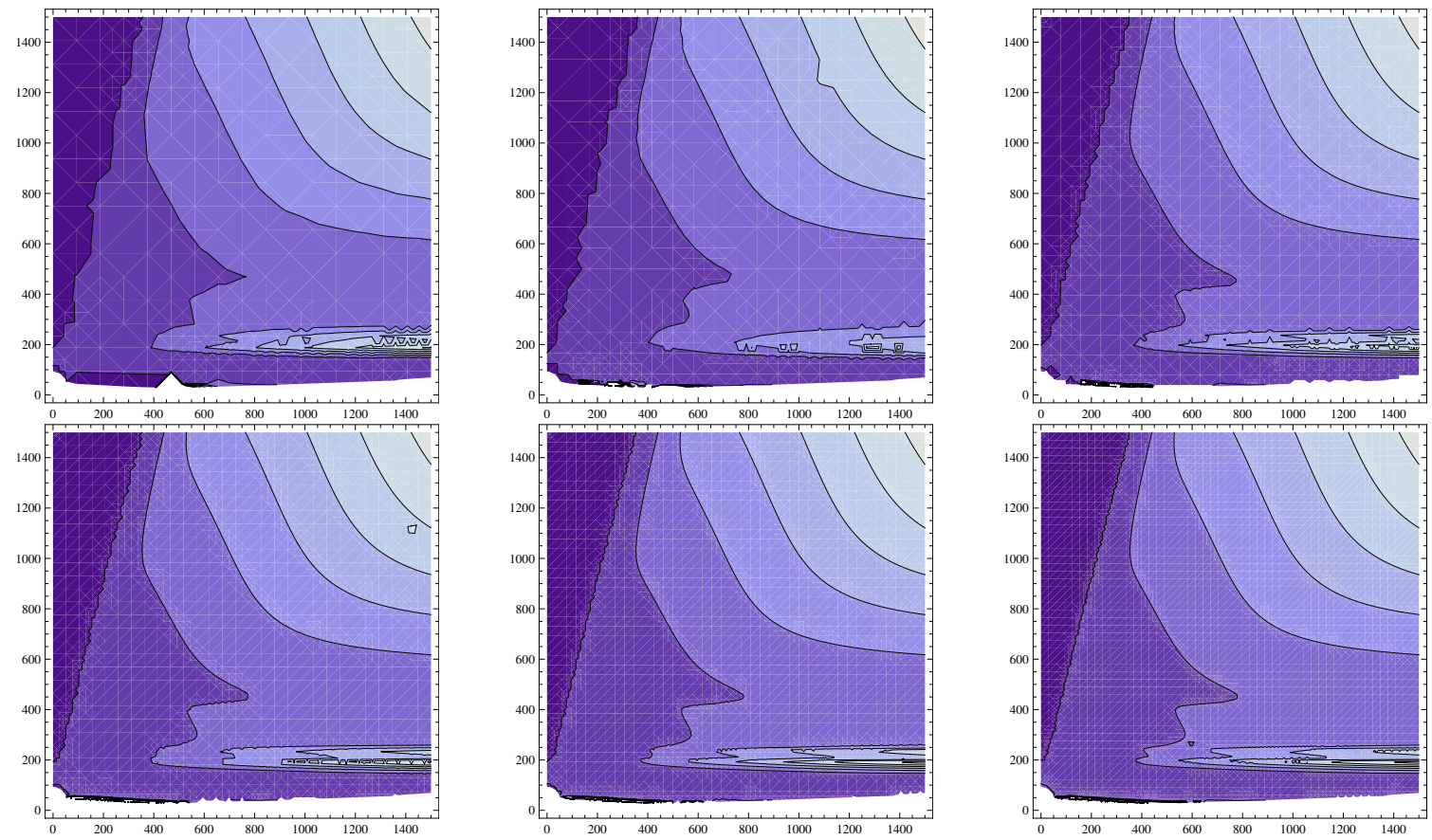

Figure 3: $\left(m_{0}, M_{1 / 2}\right)$ plane for different values of PlotPoints. First row (from left to right): 5 , 10, 20. Second row (from left to right): 30, 40,50. The mesh gives an insight, where Mathematica has decreased the distance between the interpolation points. 


\begin{tabular}{|c|c|c|c|c|c|c|}
\hline PlotPoints & 5 & 10 & 20 & 30 & 40 & 50 \\
\hline Calculated points & 990 & 2077 & 4589 & 9242 & 13324 & 17416 \\
\hline
\end{tabular}

Table 3: Number of evaluated parameter space points in the $\left(m_{0}, M_{1 / 2}\right)$-plane for a given value of PlotPoints.

\section{Putting the programs to work}

\subsection{Automated setup and model implementation}

In order to simplify the setup of the different programs and the generated model files, we provide a script to setup and install the complete environment and another script for the automated implementation of new models. These scripts can be downloaded from

http://projects.hepforge.org/sarah/Toolbox.html

After downloading the current version of the package, untar it using

$>$ tar -xzf toolbox-\$Version.tar.gz

To download and install the different packages, create and change to a working directory (which will later contain the installed packages) and call the configure script from there, e.g.

$>$ mkdir build

$>$ cd build

$>$../configure

The configure script will now proceed to check for the requirements of the different packages and download any missing files. All downloaded archives will be placed in the tarballs subdirectory of the directory containing the configure script from where they will be reused in all subsequent runs. Command line options can be used to disable specific packages and to point the script to custom locations of compilers and of the Mathematica kernel; a full list of those can be obtained by calling configure with the --help option.

After configure finishes successfully, make can be called to build all configured packages

$>$ make

After make has finished, the different packages are ready to use and can be found in subdirectories of the build directory, together with a suitable setup file for SSP.

Also created by configure is a script which automates the implementation of a new models into the different packages

$>$./butler [model]

where [model] is the models' SARAH name. For instance,

$>$./butler NMSSM

resp. 
>./ImplementModel.sh OmegaShort/Regime-2

can be used to install the NMSSM / resp. the left-right symmetric model discussed in sec. A.2. A list of different command line options can be obtained with

$>$. /butler --help

After butler has completed, the new model is implemented into all selected packages and ready for use.

\subsection{Preparing a model by hand}

If more flexibility is required than is offered by butler, the model files must be generated and integrated into the various packages by hand. In order to demonstrate how the corresponding workflow looks like, we show how the NMSSM version included in SARAH is implemented into the other programs ${ }^{2}$.

In order to implement the MSSM and prepare for an automatic parameter scan using SSP, the following steps are necessary

1. Prepare SARAH and generate all output

(a) Define the model in SARAH and evaluate it

$$
\begin{aligned}
& \text { In [1] : }<\text { SARAH.m; } \\
& \text { In [2]: }<\text { Start ["NMSSM"]; }
\end{aligned}
$$

(b) Create the source code for SPheno

In [ 3] : MakeSPheno [ ]

(c) Create model files for CalcHep and micromeGAs

In [6]: MakeCHep [ ] ;

(d) Create model files for WHIZARD

In [ 5] : MakeWHIZARD [ ] ;

The last three steps can also be done at once by using MakeAll[]. This produces the SARAH output for SPheno, CalcHep, WHIZARD, FeynArts and LATEX.

2. Prepare SPheno

(a) Create a subdirectory in your installation of SPheno3.1 or later and copy the gnerated code there

$>$ cd [SPheno Directory]/

$>\operatorname{mkdir} \mathrm{NMSSM}$

$>\mathbf{c p}$ [SARAH Directory]/Output/NMSSM/EWSB/SPheno/* ।

[SPheno Directory] /NMSSM/

\footnotetext{
${ }^{2}$ For the details on the installation of the different packages we refer to the respective documentation of the programs.
} 
(b) Compile SPheno

$>$ make Model=NMSSM

3. Prepare CalcHep

(a) Create a new project in CalcHep

$>$ cd [CalcHep Directory]/

$>$./mkUsrDir NMSSM

(b) Copy the model files from SARAH to CalcHep

$>$ cp [SARAH Directory]/Output/NMSSM/EWSB/CHep/* ।

[CalcHep Directory]/NMSSM/models/

(c) Start CalcHep and create an executable $n_{-}$calchep program for a specific process using make $\mathrm{n}_{-} \mathrm{calchep}$

$>$ cd NMSSM/

$>$./calchep

(d) Copy $n_{-}$calchep and the other created files including the subdirectory aux to a new directory

$>\mathbf{c p}-\mathrm{R}$ results/* [Process Directory]/

(e) Repeat the last two steps for other processes if necessary

4. Prepare micromegAs

(a) Create a new project in micrOMEGAs

$>$ cd [Micromegas Directory]

$>$./newProject NMSSM

(b) Copy the CalcHep model files to the project directory

> cp [SARAH Directory]/Output/NMSSM/EWSB/CHep/* ।

[MicrOmegas Directory]/NMSSM/work/models/

(c) Move the micrOMEGAs main file to the main directory and compile it

$>$ cd [Micromegas Directory]/NMSSM/

$>\mathbf{m v}$ work/models/Calcomega.cpp.

$>$ make main=Calcomega.cpp

5. Prepare WHIZARD

(a) Configure the model source

> cd [SARAH Directory]/Output/NMSSM/EWSB/WHIZARD_Omega/

$>$./configure WO_CONFIG=[WHIZARD Directory]/bin/ 
(b) Compile and install the new model (with this configuration, the files will end up in \$HOME/. whizard)

$>$ make install

\subsection{Running the chain}

Now, everything is in its place, and the model can be used with the different programs:

- Go the the SPheno directory, edit the LesHouches file and run SPheno

$>$ cd [SPheno Directory]/NMSSM/

$>$ edit LesHouches.in.NMSSM

$>$. /./bin/SPhenoNMSSM

Make sure that the flags

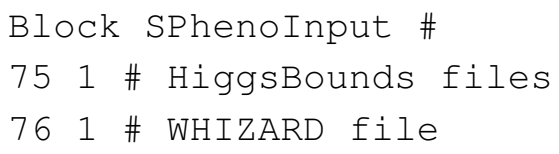

are set in LesHouches.in. nMSSM in order to create the parameter file for WHIZARD as well as the input files for HiggsBounds.

- Copy the spectrum file to the micrOMEGAs directory and the file for WHIZARD to your WHIZARD working directory

$>$ cp SPheno.spc.NMSSM [Micromegas Directory]/NMSSM/

$>\mathbf{c p}$ WHIZARD.par.NMSSM [WHIZARD Working Directory]/

- Run micromeGAs

$>$ cd [Micromegas Directory]/NMSSM/

$>$. / Calcomega

- Create a WHIZARD input file (e.g Input.sin) with your process setup and include the parameter file of SPheno by using Include ("WHIZARD.par.NMSSM") .

- Run WHIZARD

$>$ whizard Input.sin

- Copy the SPheno spectrum files to the directory of your CalcHep executables and run $n_{-}$calchep

$>\mathbf{c p}[$ SPheno Directory]/bin/SPheno.spc.NMSSM [CH Process Directory]/

$>$ cd [CH Process Directory]/

$>$./n_calchep

To run n_calchep using a script you can also use the blind mode, see sec. 5.1. 


\section{Conclusions}

We have presented a toolchain for studying extensions of the MSSM. The model under consideration is implemented in SARAH which creates then source code for SPheno, thus facilitating the implementation of new models into SPheno in a modular way. The new SPheno modules calculate the mass spectrum using two-loop RGE running and one-loop corrections to the masses. The precision of the calculation of the mass spectrum for a large range of SUSY models is comparable to the known precision of the MSSM provided by the established spectrum generators (excluding two loop effects in the Higgs sector). Therefore, SARAH can be regarded as a 'spectrum-generator-generator', extending the availability of spectrum generators for different models dramatically.

Apart from creating output for SPheno, SARAH is capable of creating model files suitable for use with CalcHep/micrOMEGAs, FeynArts, HiggsBounds and WHIZARD. The masses and parameters calculated by SPheno can be used directly as input for those programs which then facilitate further studies of the models' phenomenology. In particular, the dark matter relic density predicted by the model can be simulated using CalcHep/ micrOMEGAs, while WHIZARD is well suited for examining collider observables involving the long decay chains typical for supersymmetric models. The scan of observables over parameter space can be automatized using the SSP package.

As SARAH works in a very generic way, a wide range of possible models is covered. Models with new fields and/or an extended gauge sector at the SUSY scale are supported as well as models with thresholds and/or gauge symmetry breaking at higher energy scales. This flexibility, together with the integration of different existing tools together with SARAH and SSP into the largely automated toolchain presented in this paper should greatly simplify the study of new supersymmetric models.

\section{Acknowledgments}

We thank for beta testing and useful feedback Martin Hirsch, Avelino Vicente, Björn Herrmann, Laslo Reichert, Benedikt Vormwald and Ben O'Leary. W.P. and F.S. have been supported by the DFG project No. PO-1337/1-1. CS has been supported by the Deutsche Forschungsgemeinschaft through the Research Training Group GRK 1102 Physics of Hadron Accelerators.

\section{A. Input files for SARAH to generate a SPheno version with thresholds}

\section{A.1 Seesaw I}

\section{Seesaw1.m}

In the case of seesaw I, the particle content is extended by three generations of a gauge singlet $\hat{\nu}_{R}$.

Fields [[8]] $=\{\mathrm{vR}, 3, \mathrm{v}, 0,1,1\} ;$ 
The new field is assigned a Yukawa like interaction and a mass term. In addition, there is an effective operator which is generated by integrating out the right handed neutrino.

SuperPotential $=$

$\{\ldots,\{\{1, \mathrm{Yv}\},\{\mathrm{v}, 1, \mathrm{Hu}\}\},\{\{1 / 2, \mathrm{Mv}\},\{\mathrm{v}, \mathrm{v}\}\},\{\{1 / 2, \mathrm{WOp}\},\{1, \mathrm{Hu}, \mathrm{l}, \mathrm{Hu}\}\}\} ;$

Since the field is heavy, it should not be included in the calculation of the vertices and masses at the SUSY scale. Therefore, we "delete" it. Note that, although deleted particles do not appear in the low energy spectrum and Feynman rules, they do contribute to the RGEs above the threshold scale.

DeleteParticles $=\{\mathrm{v}\}$;

\section{SPheno.m}

We choose a unification of the soft-breaking mass of the scalar singlet with the other soft-breaking masses at the GUT scale. In addition, we want to define the values of the superpotential parameters at the GUT scale as input values in the LesHouches file. Furthermore, we also impose a mSugra like condition for the trilinear soft-breaking coupling. As the bilinear soft-breaking term does not influence the RGE running of the other parameters, we can safely set it to zero. Altogether, the additional boundary conditions at the GUT scale are

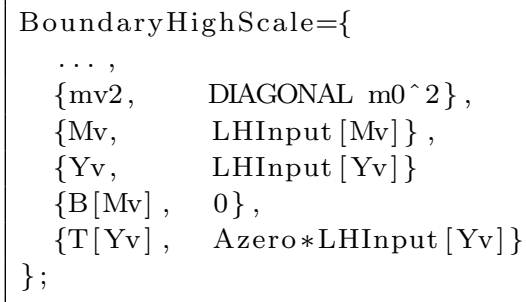

We want to include three threshold scales: each generation of the gauge singlet should be integrated out at energies similar to their mass. Therefore, a good choice is

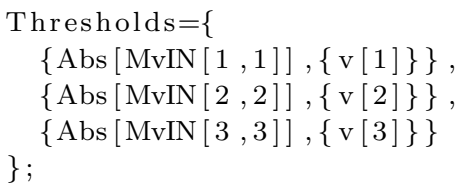

When thresholds are included, the boundary conditions for running up and down the RGEs can be defined separately for each threshold scale. To this end, it is first necessary to initialize the corresponding arrays

BoundaryConditionsUp $=$ Table $[\{\},\{$ Length [Thresholds $]\}]$;

BoundaryConditionsDown=Table $[\{\},\{$ Length [Thresholds $]\}]$;

When a gauge singlet is integrated out, the Wilson coefficient of the effective dim-5 operator receives a contribution of the form

$$
\kappa=-Y_{\nu}^{T} M_{N}^{-1} Y_{\nu}
$$


When heavy superfields are integrated out, the mass splitting between the fermionic and scalar component is neglected. The masses are calculated individually at each threshold scale and saved in arrays with the names

MassOf $<>$ Name of Superfield

Therefore, the contributions to the effective operator at the different scales are given by

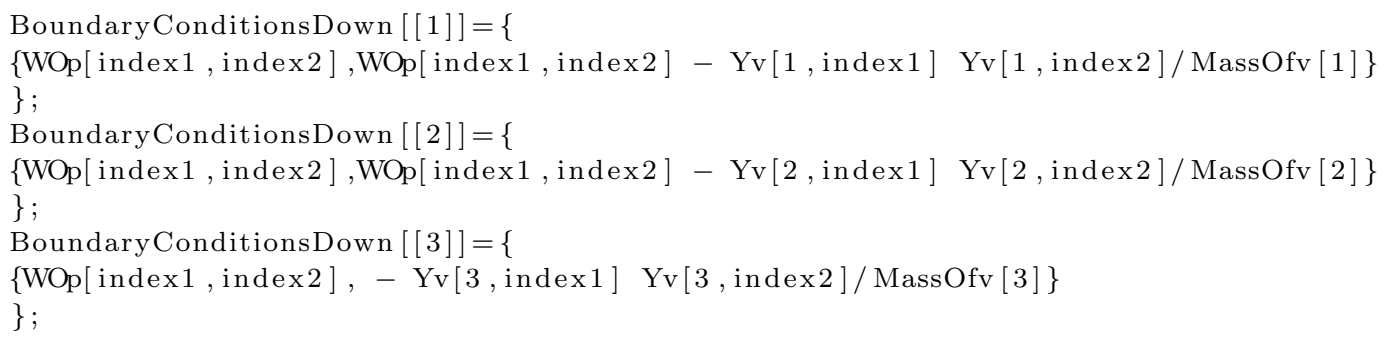

\section{A.2 Implementation of a model with a gauge symmetry breaking scale in $\mathrm{SARAH}$ and SPheno}

This section is devoted to a detailed discussion of the implementation of a left-right supersymmetric model (based on that presented in [72]) into SPheno and SARAH. Since we are mainly interested in the implementation in SARAH, it is sufficient to simplify the model a bit by just choosing one threshold scale and not two as discussed in [72]. Adding the second threshold scale is straightforward, but would lead to some redundancy in the following discussion.

\section{A.2.1 Summary of the model}

We here give only a short summary about the model and refer to [72] and references therein for more details. As adread discussed, we introduce only one threshold scale at which $\times S U(2)_{R} \times U(1)_{B-L}$ gets broken

$$
S U(2)_{L} \times S U(2)_{R} \times U(1)_{B-L} \rightarrow S U(2)_{L} \times U(1)_{Y}
$$

From GUT scale to $S U(2)_{R} \times U(1)_{B-L}$ breaking scale The MSSM particle content above the threshold is extended by the presence of four fields which are triplets under $S U(2)_{L}$ or $S U(2)_{R}$ and which carry a $B-L$ charge. In addition, there are two triplets which are uncharged under $B-L$. Furthermore, the right handed neutrino are part of the spectrum and the Higgs fields are arranged in so-called bi-doublets $\Phi$. To get a non-trivial CKM matrix, we need at least two generations of $\Phi$ fields. The particle content can be summarized as follows 


\begin{tabular}{cccccc}
\hline Superfield & generations & $S U(3)_{c}$ & $S U(2)_{L}$ & $S U(2)_{R}$ & $U(1)_{B-L}$ \\
\hline$Q$ & 3 & 3 & 2 & 1 & $\frac{1}{3}$ \\
$Q^{c}$ & 3 & $\overline{3}$ & 1 & 2 & $-\frac{1}{3}$ \\
$L$ & 3 & 1 & 2 & 1 & -1 \\
$L^{c}$ & 3 & 1 & 1 & 2 & 1 \\
$\Phi$ & 2 & 1 & 2 & 2 & 0 \\
$\Delta$ & 1 & 1 & 3 & 1 & 2 \\
$\bar{\Delta}$ & 1 & 1 & 3 & 1 & -2 \\
$\Delta^{c}$ & 1 & 1 & 1 & 3 & -2 \\
$\bar{\Delta}^{c}$ & 1 & 1 & 1 & 3 & 2 \\
$\Omega$ & 1 & 1 & 3 & 1 & 0 \\
$\Omega^{c}$ & 1 & 1 & 1 & 3 & 0 \\
\hline
\end{tabular}

The superpotential for the model reads

$$
\begin{aligned}
\mathcal{W} & =Y_{Q} Q \Phi Q^{c}+Y_{L} L \Phi L^{c}-\frac{\mu}{2} \Phi \Phi+f L \Delta L+f^{*} L^{c} \Delta^{c} L^{c} \\
& +a \Delta \Omega \bar{\Delta}+a^{*} \Delta^{c} \Omega^{c} \bar{\Delta}^{c}+\alpha \Omega \Phi \Phi+\alpha^{*} \Omega^{c} \Phi \Phi \\
& +M_{\Delta} \Delta \bar{\Delta}+M_{\Delta}^{*} \Delta^{c} \bar{\Delta}^{c}+M_{\Omega} \Omega \Omega+M_{\Omega^{*}}^{*} \Omega^{c} .
\end{aligned}
$$

Below $S U(2)_{R} \times U(1)_{B-L}$ breaking scale Here, we are left with the MSSM plus the effective Weinberg operator which causes neutrino masses after EWSB.

Boundary conditions In order to link both scales, we need the following set of boundary conditions:

$$
\begin{array}{ll}
Y_{d}=Y_{Q}^{1} \cos \theta_{1}-Y_{Q}^{2} \sin \theta_{1}, & Y_{u}=-Y_{Q}^{1} \cos \theta_{2}+Y_{Q}^{2} \sin \theta_{2}, \\
Y_{e}=Y_{L}^{1} \cos \theta_{1}-Y_{L}^{2} \sin \theta_{1}, & Y_{\nu}=-Y_{L}^{1} \cos \theta_{2}+Y_{L}^{2} \sin \theta_{2},
\end{array}
$$

where $R=\sin \left(\theta_{1}-\theta_{2}\right)$. For the soft-trilinear couplings, $Y$ is replaced with $T$ in the expressions. For the sfermionic soft masses, we have

$$
\begin{aligned}
m_{q}^{2}=m_{u^{c}}^{2} & =m_{d^{c}}^{2}=m_{Q^{c}}^{2}, \\
m_{l}^{2} & =m_{e^{c}}^{2}=m_{L^{c}}^{2}, \\
M_{L} & =M_{R}=M_{2} .
\end{aligned}
$$

while in the Higgs sector we need the relations

$$
\begin{aligned}
& m_{H_{d}}^{2}=\cos ^{2} \theta_{1}\left(m_{\Phi}^{2}\right)_{11}+\sin ^{2} \theta_{1}\left(m_{\Phi}^{2}\right)_{22}-\sin \theta_{1} \cos \theta_{1}\left[\left(m_{\Phi}^{2}\right)_{12}+\left(m_{\Phi}^{2}\right)_{21}\right], \\
& m_{H_{u}}^{2}=\cos ^{2} \theta_{2}\left(m_{\Phi}^{2}\right)_{11}+\sin ^{2} \theta_{2}\left(m_{\Phi}^{2}\right)_{22}-\sin \theta_{2} \cos \theta_{2}\left[\left(m_{\Phi}^{2}\right)_{12}+\left(m_{\Phi}^{2}\right)_{21}\right],
\end{aligned}
$$

In the gauge sector, we have to express the hypercharge coupling and the corresponding gaugino through

$$
\begin{aligned}
g_{1} & =\frac{\sqrt{5} g_{2} g_{B L}}{\sqrt{2 g_{2}^{2}+3 g_{B L}^{2}}}, \\
M_{1} & =\frac{2 g_{2}^{2} M_{B L}+3 g_{B L}^{2} M_{R}}{2 g_{2}^{2}+3 g_{B L}^{2}} .
\end{aligned}
$$




\section{A.2.2 Model files for SARAH}

We now discuss the input files for SARAH required for defining the model at the different scales. For shortness, we concentrate on the parts necessary for SPheno output and skip the gauge fixing terms and definition of Dirac spinors above the threshold scale.

\section{From GUT scale to $S U(2)_{R} \times U(1)_{B-L}$ breaking scale}

The vector and chiral superfields at the highest scale defining the gauge sector and particle content are defined by

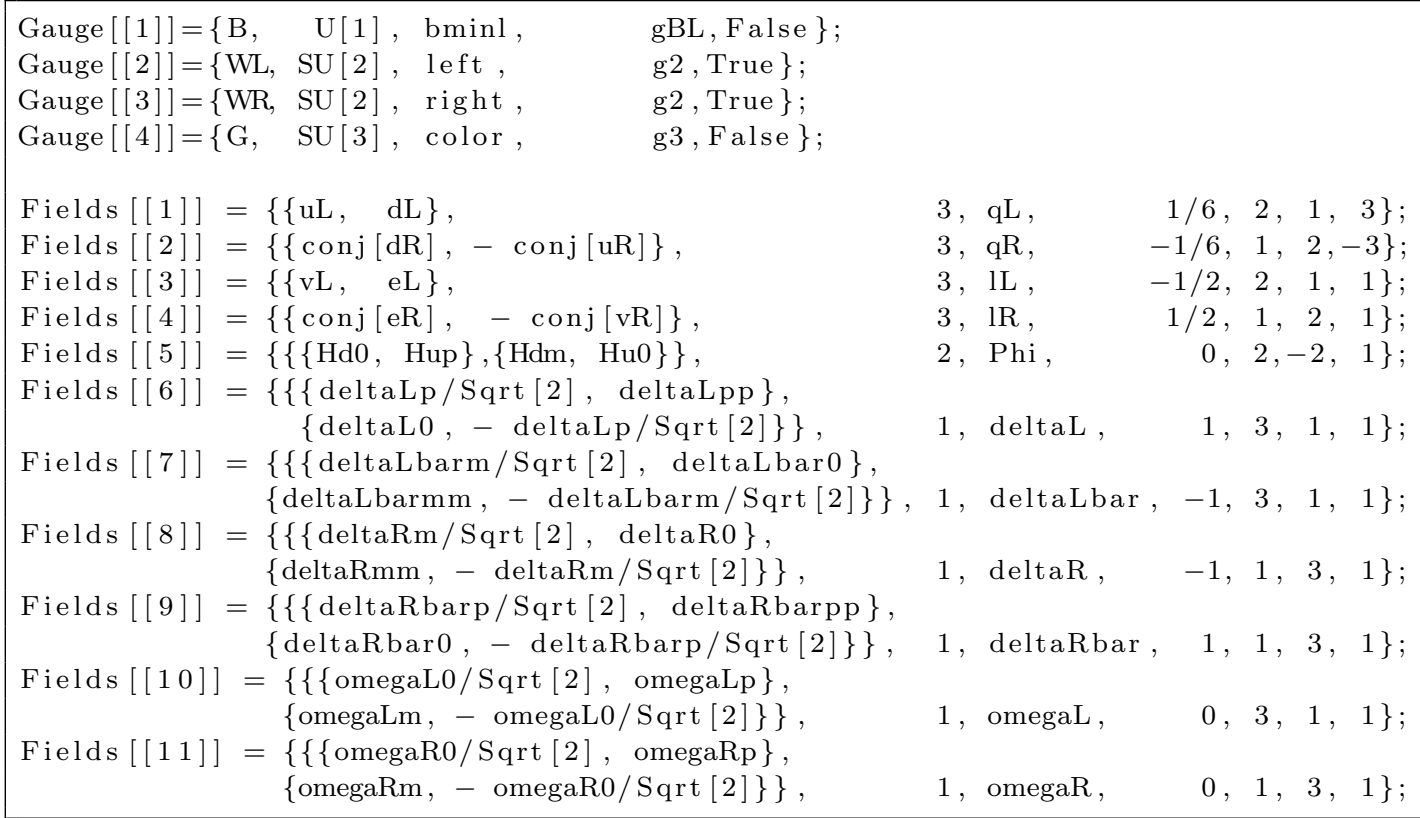

The superpotential reads

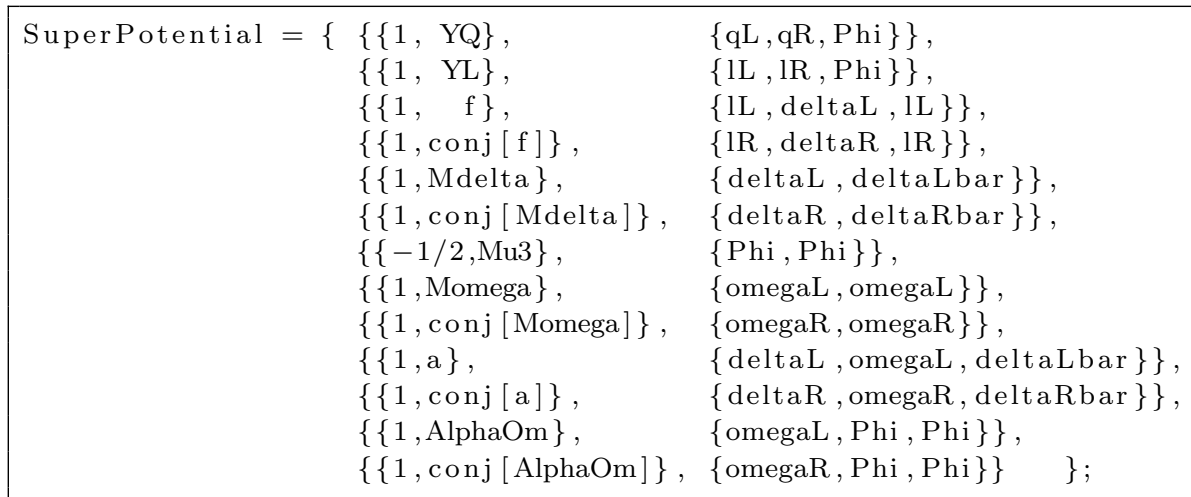

The gauge bosons and gauginos of the right sector decompose into

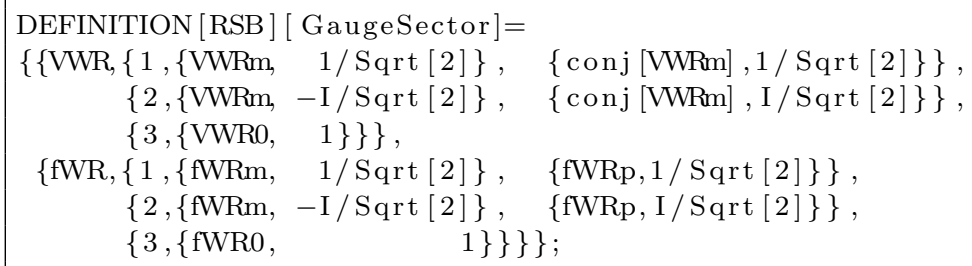


after the $\Omega$ and $\Delta$ fields have received their VEV

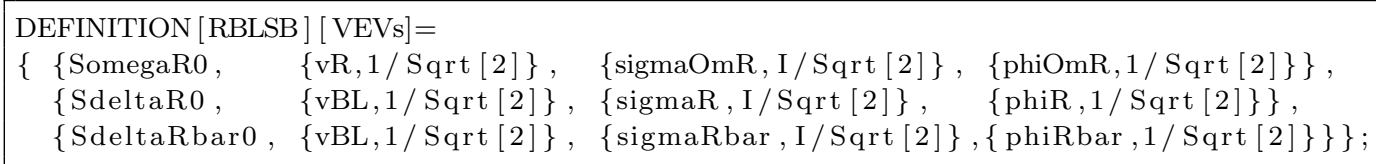

Finally, we need the rotations in the matter sector to the new mass eigenstates

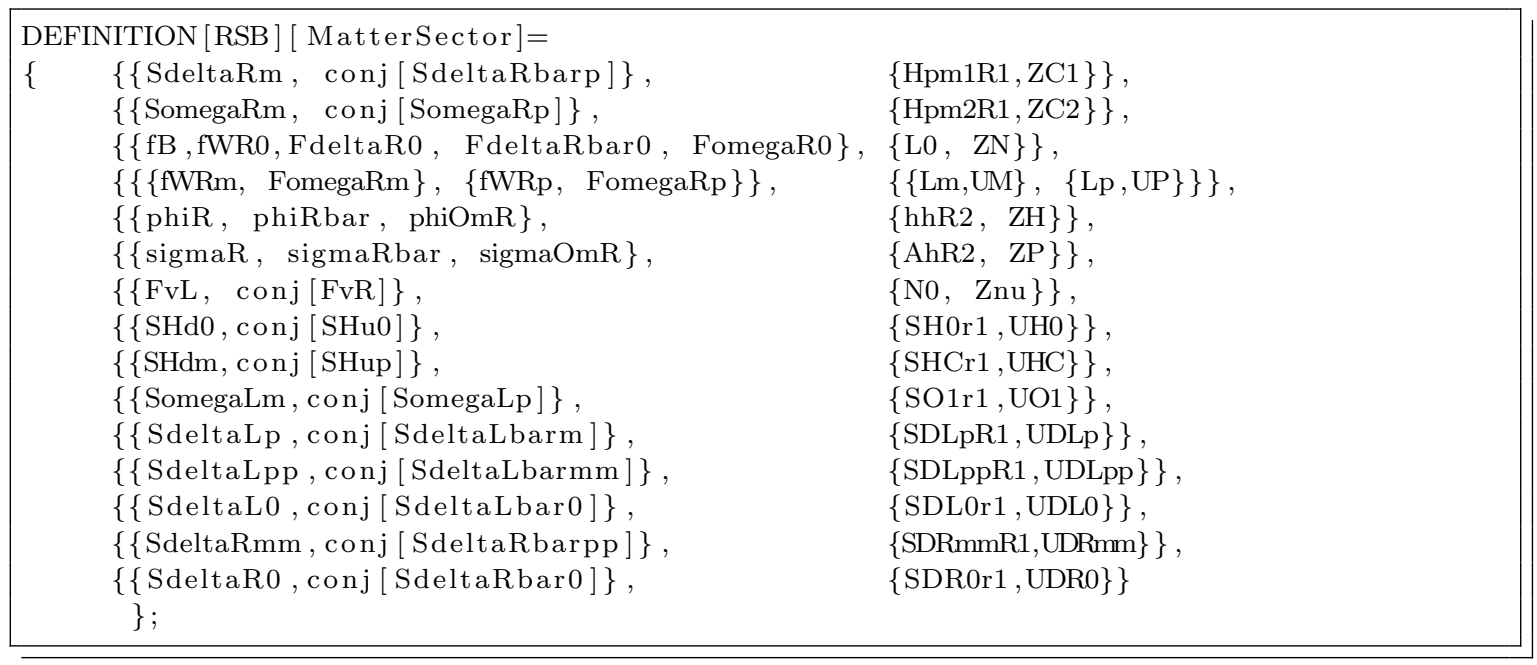

Below $S U(2)_{R} \times U(1)_{B-L}$ breaking scale

Below the breaking scale, only the particles and gauge groups of the MSSM survive as dynamical degrees of freedom. Therefore, the model file is almost identical that for the MSSM. We only point out the differences:

- The superpotential contains the Weinberg operator

SuperPotential $=\{\ldots,\{\{1, \mathrm{WOp}\},\{1, \mathrm{Hu}, \mathrm{l}, \mathrm{Hu}\}\} \quad\}$

- The neutrinos are massive and mix among each other

DEFINITION $[$ EWSB] $[$ MatterSector $]=\{\ldots,\{\{\mathrm{FvL}\},\{\mathrm{FV}, \mathrm{UV}\}\}\}$;

- These states form Majorana spinors

$\operatorname{dirac}[[4]]=\{\mathrm{Fv}, \quad \mathrm{FV}, \operatorname{conj}[\mathrm{FV}]\}$

\section{A.2.3 Model files for SPheno output}

Two SPheno.m files, one for each scale range, are neccessary in order for SARAH to create the Fortran source code. While the first one is rather short, the second one includes all necessary boundary conditions. 


\section{Above $S U(2)_{R} \times U(1)_{B-L}$ breaking scale}

First, the regime must be flagged as an intermediate scale, and SARAH must be told its position in the scale hierarchy (counted from GUT to low scale).

RegimeNr $=1$;

IntermediateScale $=$ True;

Afterwards, we give a list with all particles which are integrated out at the threshold scale after gauge symmetry breaking.

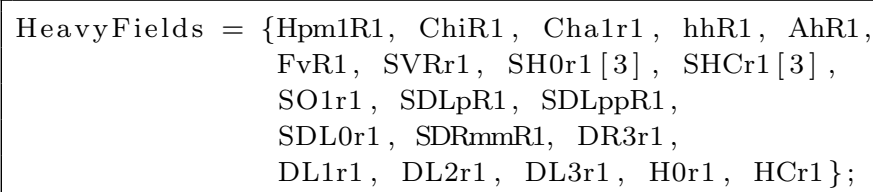

The numbers in square brackets indicate that only the third generation and above is integrated out.

In order to calculate the finite shifts of the gauge couplings and gaugino masses, it is necessary to define the gauge sector of the next scale NextGauge as well as the quantum number of the fields which are integrated out with respect to those gauge groups.

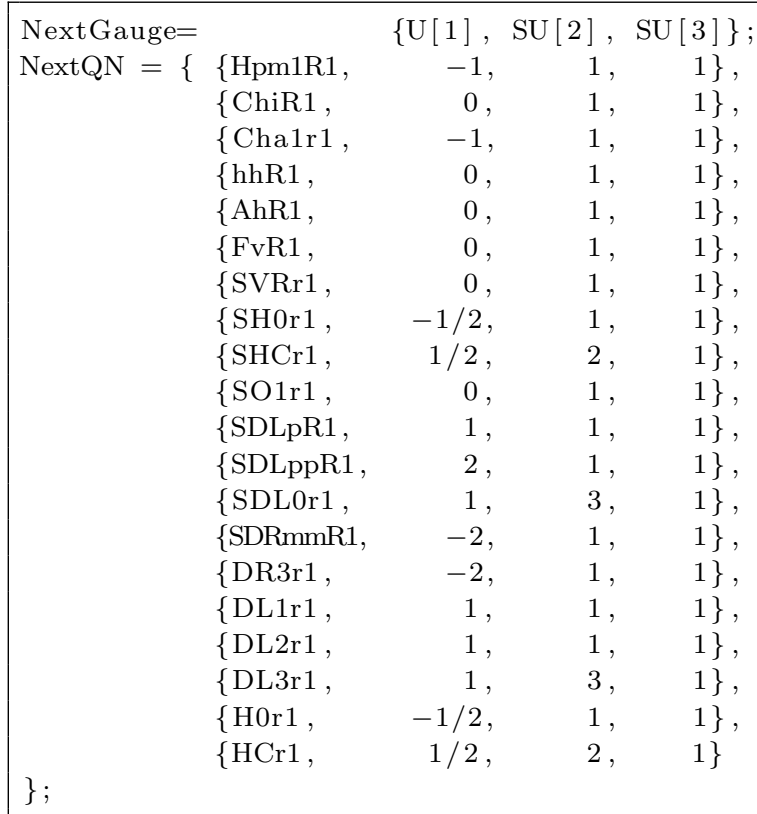

Finally, SARAH needs information on the vacuum conditions. There are two different VEVs, and therefore, we need to choose two parameters which are fixed by the tadpole equations. As there is no closed analytical solution for them, we give an approximation obtained by neglecting the soft-breaking terms.

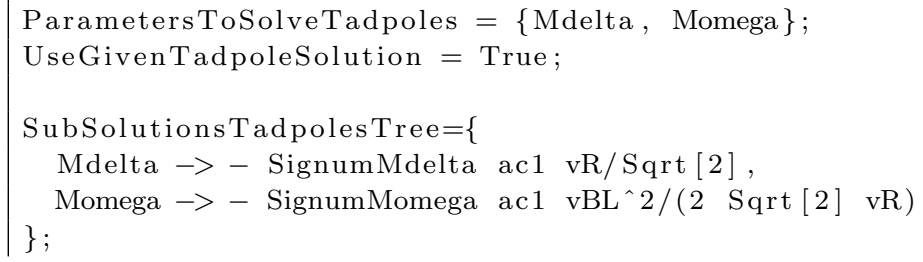




\section{Below $S U(2)_{R} \times U(1)_{B-L}$ breaking scale}

The second scale is not an intermediate scale, and hence

RegimeNr $=2$;

IntermediateScale $=$ False

We make the following choice of free parameters of that model: to the set of standard mSugra parameters $\left(m_{0}, M_{1 / 2}, A_{0}, \tan \beta, \operatorname{sign} \mu\right)$, we add $B_{0}$, the superpotential parameter $a$, the signs of $M_{\Omega}$ and $M_{\Delta}$, the two VEVs $v_{R}$ and $v_{B L}$, and the threshold scale. These are defined in the blocks MINPAR and EXTPAR.

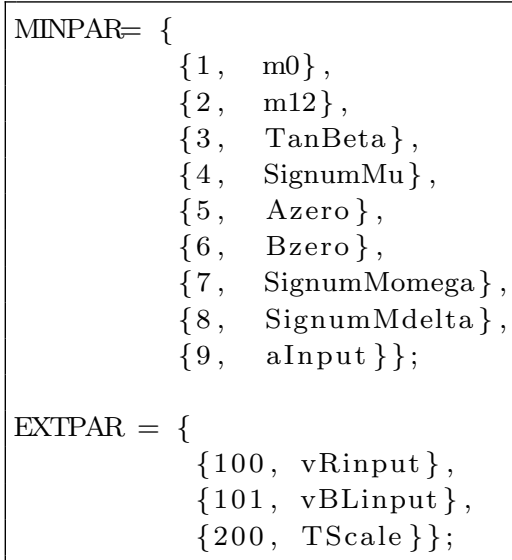

As in the, MSSM we fix the numerical values of $\mu$ and $B_{\mu}$ by the solving the two tadpole equations,

ParametersToSolveTadpoles $=\{\backslash[\mathrm{Mu}], \mathrm{B}[\backslash[\mathrm{Mu}]]\}$;

Furthermore, we use also the common definitions for the SUSY scale, see also sec. 3.5.

RenormalizationScaleFirstGuess $=\mathrm{m} 0 \wedge 2+4 \mathrm{~m} 12^{\wedge} 2$;

Renormalizationscale $=\operatorname{MSu}[1] * \operatorname{MSu}[6]$;

We use as condition for the GUT scale

ConditionGUTscale $=\{\mathrm{gBL}=\mathrm{g} 2, \mathrm{~g} 1=\mathrm{g} 2\}$;

The second entry is necessary because it might be that the masses of the fields breaking the left-right symmetry are above the unification scale which has to be handled seperatly. At the GUT scale we use boundary conditions motivated by minimal supergravity: all scalar soft-breaking masses are proportional to $m_{0}$, the gaugino masses are proportional to $M_{1 / 2}$, the trilinear soft-breaking couplings are given by the corresponding superpotential parameter times $A_{0}$, and the bilinear soft-breaking couplings are set to $B_{0}$ times the superpotential parameter. The values for the coupling matrices $f, \alpha$ as well as for $\mu$ are read from the LesHouches input file.

BoundaryHighScale $=\{$

$\{\mathrm{T}[\mathrm{YQ}], \quad$ Azero $* \mathrm{YQ}\}$, 


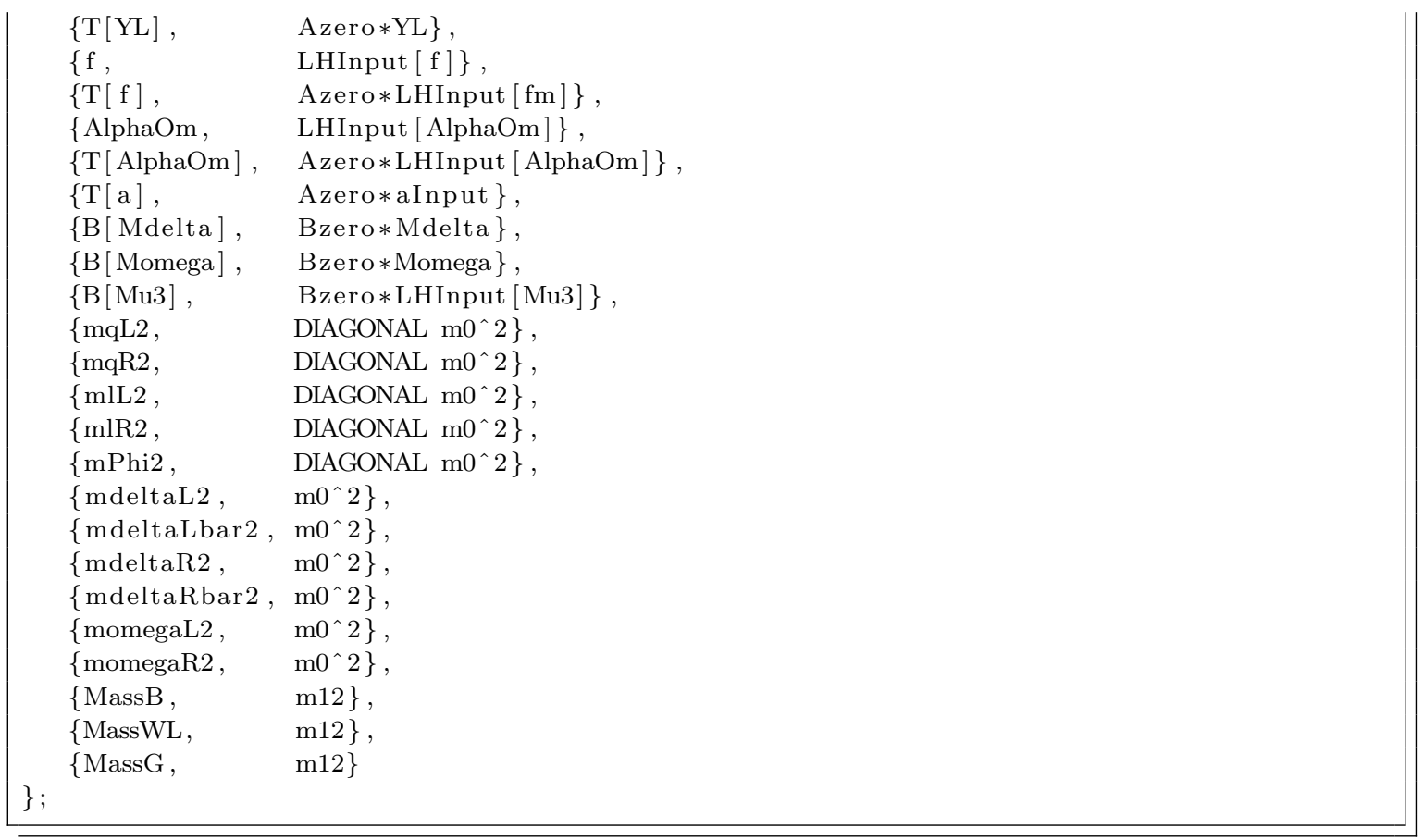

To glue the both regimes, we need to define the appropriate boundary conditions. First, we initialize the arrays

ThresholdScales $=\{$ TSCALE $\}$

BoundaryConditionsUp $=$ Table $[\{\},\{$ Length [ThresholdScales $]\}] ;$

BoundaryConditionsDown $=$ Table $[\{\},\{$ Length $[$ ThresholdScales $]\}]$;

and then encode the equations eqs. (A.4)-(A.12). In order to keep the code short, we define

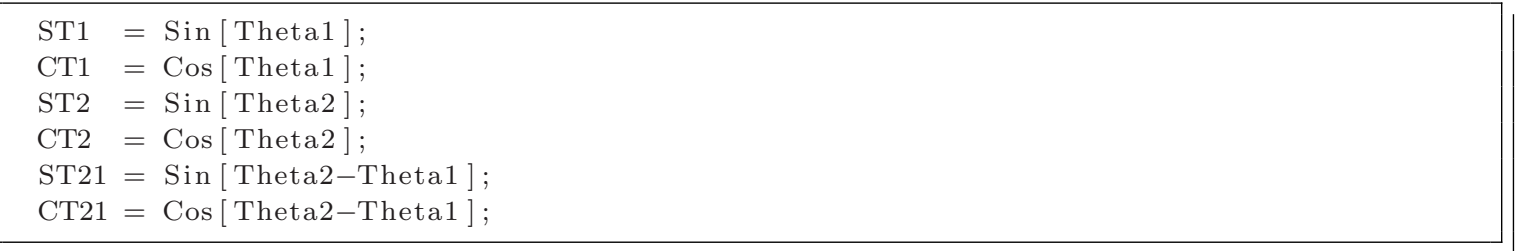

Using these abbreviations, the boundary conditions can be written as

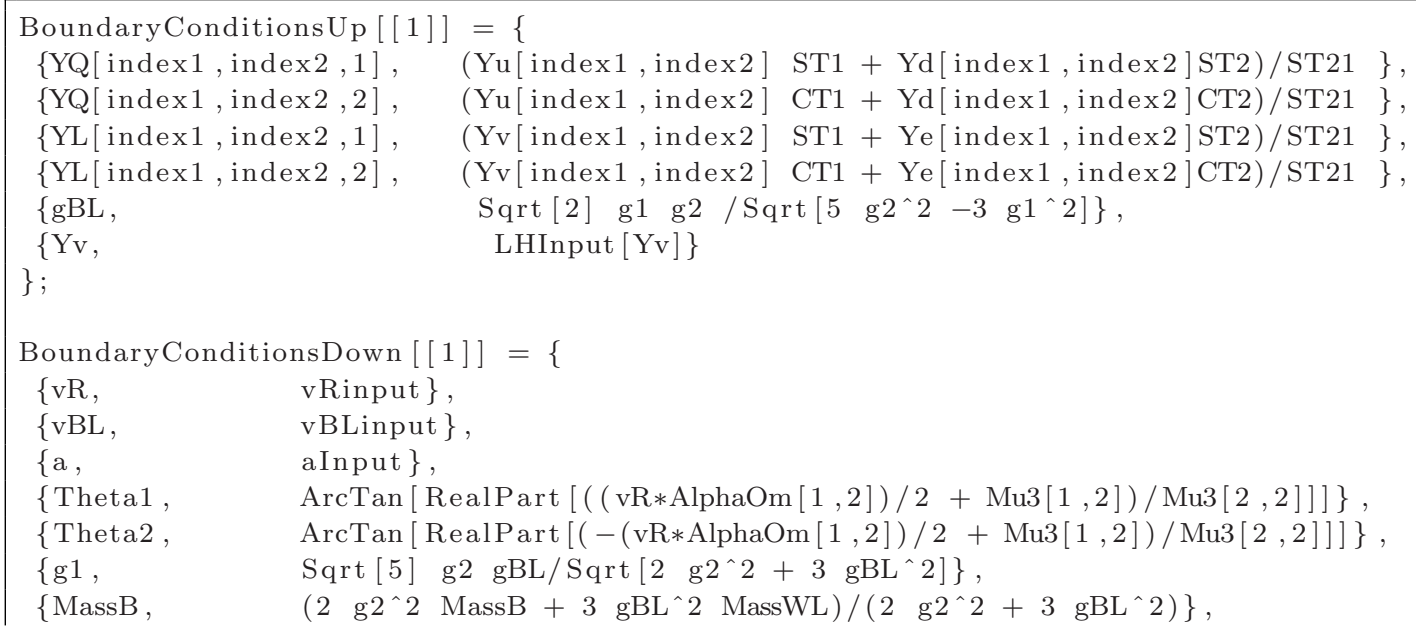




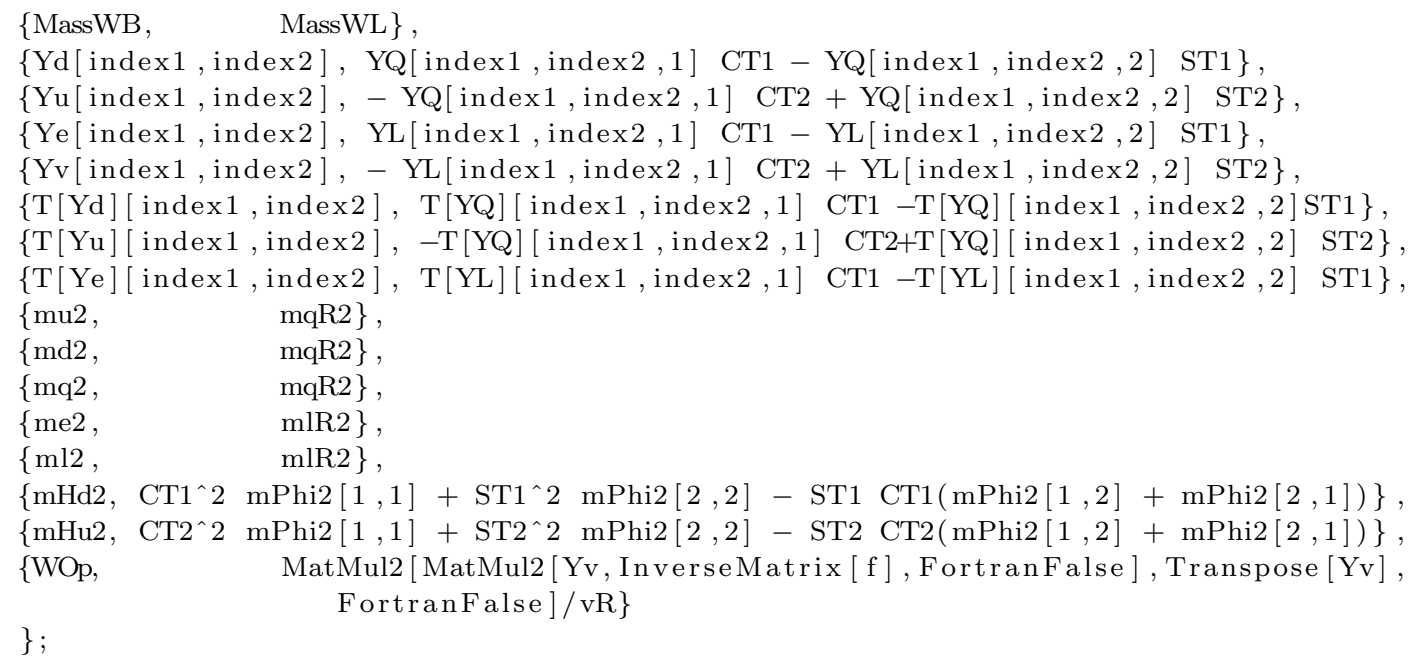

Note that $Y_{\nu}$ does not appear in any of the model files and it is therefore necessary to fix the dimension of that matrix by hand

AdditionalVariablesSPheno $=\{\mathrm{Yv}[3,3]\}$;

Several parameters are restricted to be real. In addition, it is helpful to choose initialization values for some parameters to stabilize the numerics in the first iteration

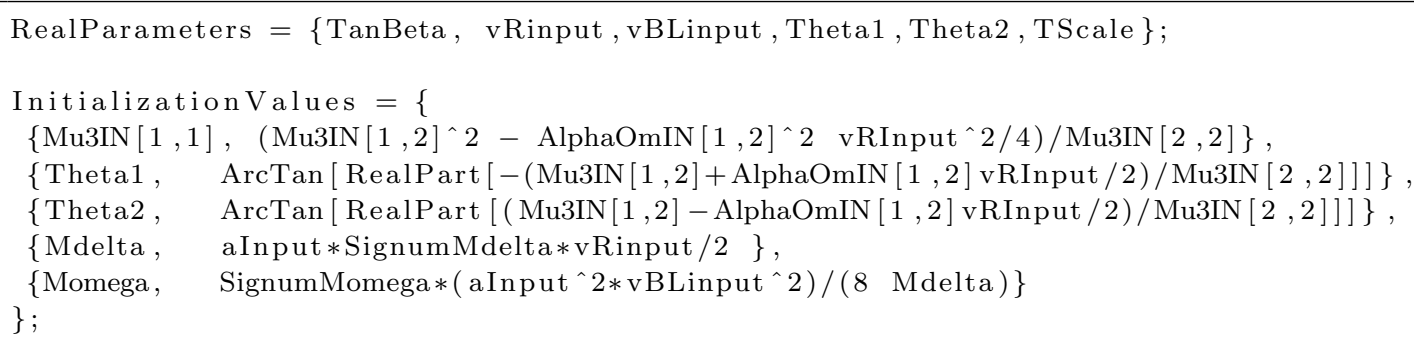

\section{B. Example for using SSP in the MSSM: $\left(m_{0}, M_{1 / 2}\right)$ grid scan}

In this section we give an example on how to generate scans and plots within in the MSSM. The example shown here is a short version of the file Example1.m included in SSP. The goal will be varying $m_{0}$ and $M_{1 / 2}$ between 0 and $1000 \mathrm{GeV}$ and thus investigating how different masses depend on these two parameters.

First, it is necessary to define the location of all tools ${ }^{3}$ in a file called Default Settings.m. The content of Defaultsettings.m should look like

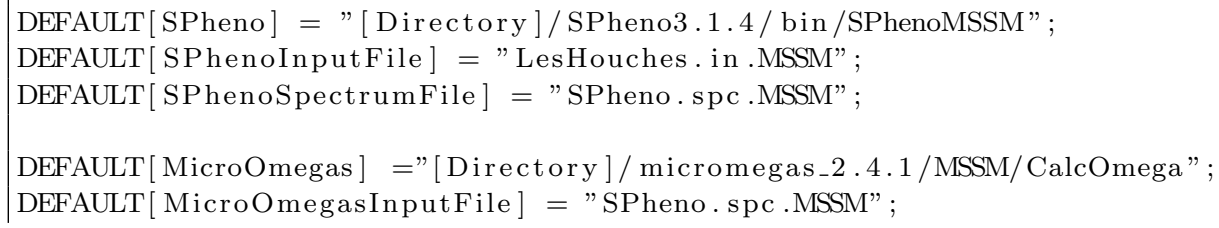

${ }^{3}$ If the environment is set up using our provided script (c.f. 8.1), the necessary file is created automatically in the process. 
DEFAULT[ MicroOmegasOutputFile] = "omg.out";

DEFAULT [WHIZARD] = "[Directory $] /$ whizard/bin/whizard" ;

DEFAULT [WHIZARDparFile] = "WHIZARD. par.MSSM";

DEFAULT[HiggsBounds] = "[Directory]/HiggsBounds/HiggsBounds LandH effC $31 "$;

[Directory] stands for the installation directory of the framework presented here. In principle, for this example it would have been sufficient to set only the information for SPheno.

The properties of the desired scan have to be defined in a second input file located in SSP / Input which we will call Exampple_MSSM.m. The first thing to into the file is a list with identifiers for the different scans, providing the possibility to perform several scans in a row using only one input file. Here, we will restrict ourself to just one scan

RunScans $=\{$ ScanM0M12 $\}$;

In order to actually perform the scans, the necessary information for creating the LesHouches input has to be provided, which comprises all appearing block names as well as numerical values for the parameters.

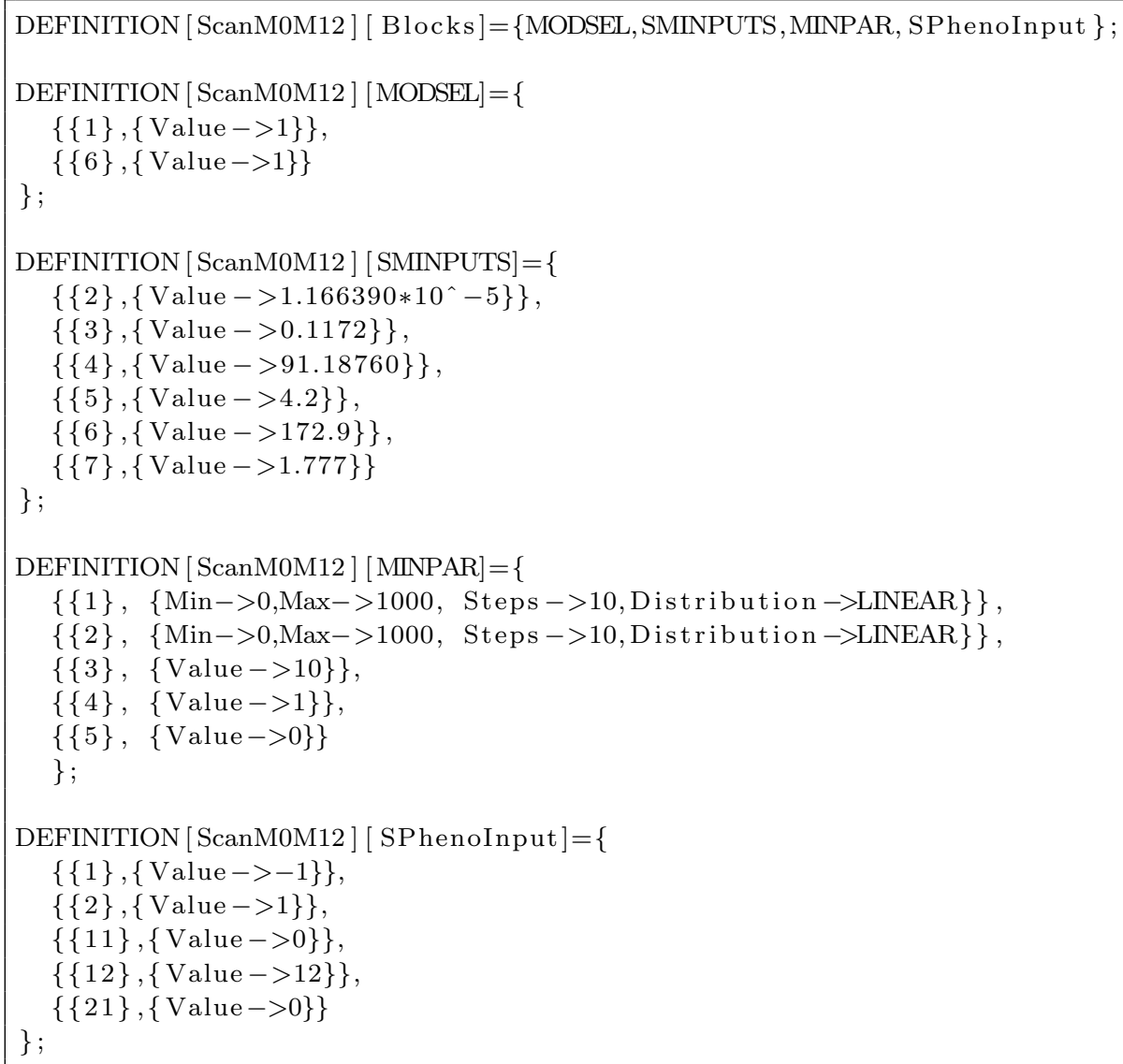

Observe that all parameters are assigned constant value, except $m_{0}$ and $M_{1 / 2}$ which are to be linearly varied.

Finally, we define the plot variables. In this example, we choose 
- All three neutral Higgs masses for different $m_{0}$ as functions of $M_{1 / 2}$

- All six selectron masses for different $m_{0}$ as functions of $M_{1 / 2}$

- All four neutralino masses for different $M_{1 / 2}$ as functions of $m_{0}$

- All three neutral Higgs masses for different $M_{1 / 2}$ as functions of $m_{0}$

- Contour plot of the light Higgs mass in the $\left(m_{0}, M_{1 / 2}\right)$-plane

- Contour plot of the heavy Higgs mass in the $\left(m_{0}, M_{1 / 2}\right)$-plane

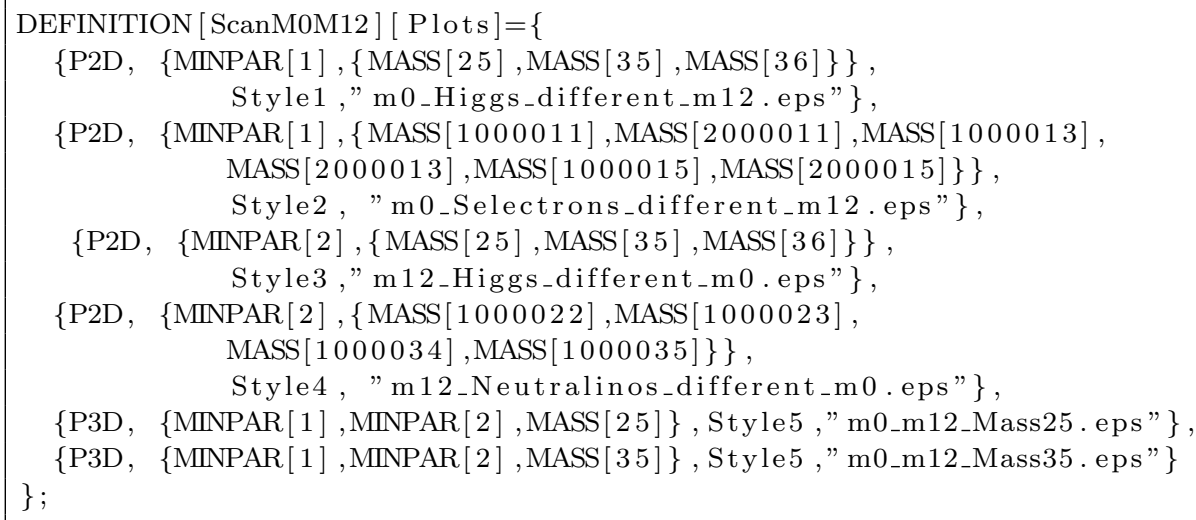

Finally, we polish the plots by adjusting Mathematica's plot options

Style $5=\{$ Frame $\rightarrow$ True, Axes $\rightarrow$ False,

FrameLabel $\rightarrow$ \{Style [Subscript ["m","0"],16], Style [Subscript ["M","1/2"],16]\},

FrameTicksStyle $\rightarrow$ Directive [Black, 14], ContourLabels $\rightarrow$ True $\}$;

For a detailed description of those options, we refer to the manual of Mathematica. The final plots are shown in Fig. 4. 

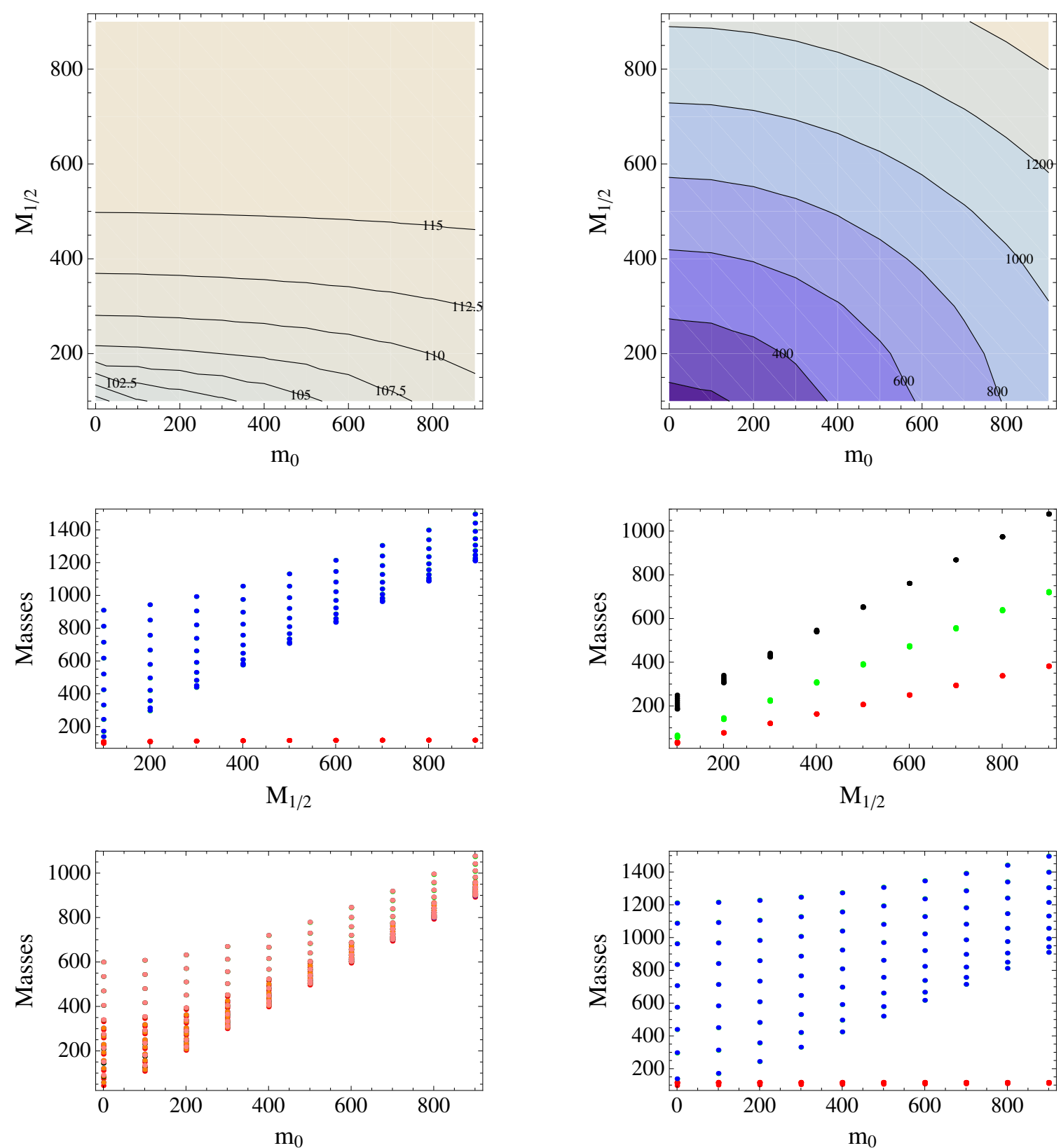

Figure 4: From top to bottom, left to right: mass of the light Higgs in the $\left(m_{0}, M_{1 / 2}\right)$-plane, mass of the heavy Higgs in the $\left(m_{0}, M_{1 / 2}\right)$-plane, masses of the scalar Higgs fields as functions of $M_{1 / 2}$ for different values of $m_{0}$, masses of the neutralinos as functions of $M_{1 / 2}$ for different values of $m_{0}$, masses of the heavy Higgs in the $\left(m_{0}, M_{1 / 2}\right)$ plane, masses of the scalar Higgs fields as functions of $m_{0}$ for different values of $M_{1 / 2}$, masses of the charged sleptons as functions of $m_{0}$ for different values of $M_{1 / 2}$. 


\section{Validation of SPheno output}

\section{C.1 Mass spectrum of the MSSM}

In order to demonstrate the agreement between the "stock" SPheno MSSM and the SPheno version generated by SARAH, the relative mass difference

$$
\Delta m=\frac{m_{\text {SPheno }}-m_{\text {SPheno-SARAH }}}{m_{\text {SPheno }}}
$$

for two different points as functions of $m_{0}$ resp. $M_{1 / 2}$ is shown in Fig. 5 and Fig. 6

All comparisons were done using SPheno 3.1.4 . For obtaining comparable results, the "stock" SPheno was slightly modified in order to compensate for the systematic differences shown in Tab. 1. Specifically, we restricted the calculation of the mass spectrum to twoloop RGEs and one-loop self-energies, thus neglecting the two-loop mass corrections in the Higgs sector normally included by SPheno. In addition we switched off the loop induced decays of neutralinos and gluinos. We used a relative precision of $O\left(10^{-4}\right)$ to calculate the spectrum with both SPheno versions.

\section{C.2 Decay widths and branching ratios in the MSSM}

As discussed in Sec.3, SARAH generates code for SPheno to handle all two-body decays as well as the fermionic three-body decays. Shown in is Tab. 4 is a comparison of the Higgs decays in the MSSM between SPheno and SPheno-SARAH, including all branching ratios which are larger than $10^{-4}$. A comparison of the neutralino decays into two- and threebody final states is given in Tab. 5. Furthermore, in Fig. 7 we show the relative differences between the total decay widths in SPheno "stock" vs. SPheno/SARAH as functions of $m_{0}$ for a scenario based on SPS 4.

\section{C.3 Masses in Seesaw type I - III}

In order to demonstrate that the automatic inclusion of thresholds in SARAH works and that the 1-loop boundary conditions are calculated correctly, we show a comparison for the seesaw type I-III between SPheno "stock" and SPheno/SARAH in Tab.6. The model and SPheno.m files for this scenario are shown in appendix A.1.

\section{C.4 Masses in the NMSSM}

We have compared the results of a SPheno version for the NMSSM created by SARAH with NMSSM-Tools and found good agreement. For detailed discussion including numbers, see $[55]$. 

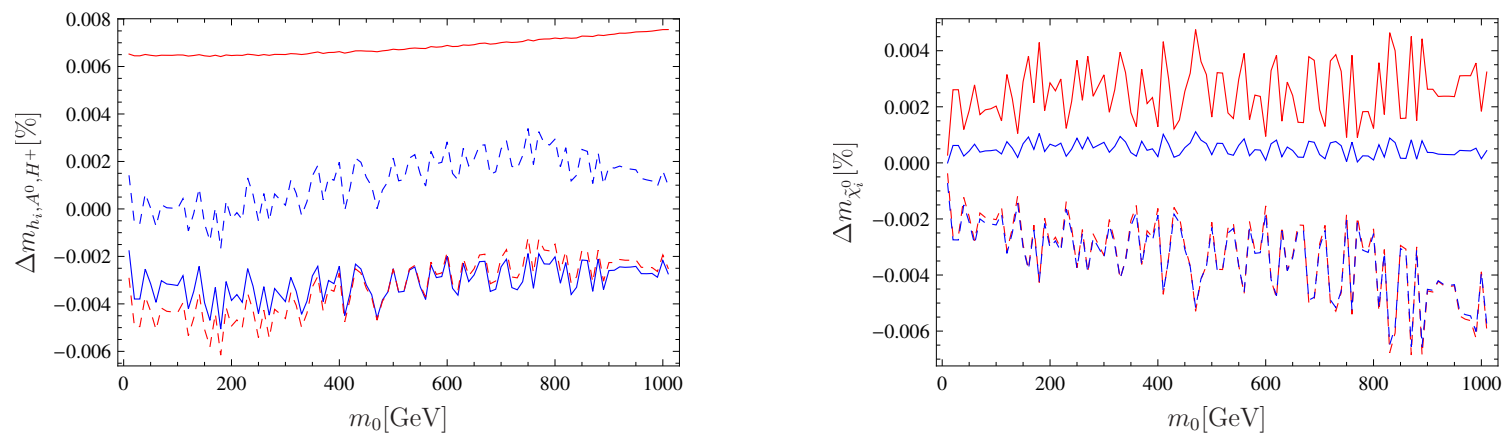

Figure 5: Comparison of the masses of the Higgs fields and neutralinos between SPheno "stock" and SPheno/SARAH. The input values are chosen as $M_{1 / 2}=500 \mathrm{GeV}, A_{0}=0 \mathrm{GeV}, \tan \beta=10$, $\operatorname{sign} \mu=1$. SPheno "stock" is based on SPheno 3.1.4 with small changes as explained in the text.
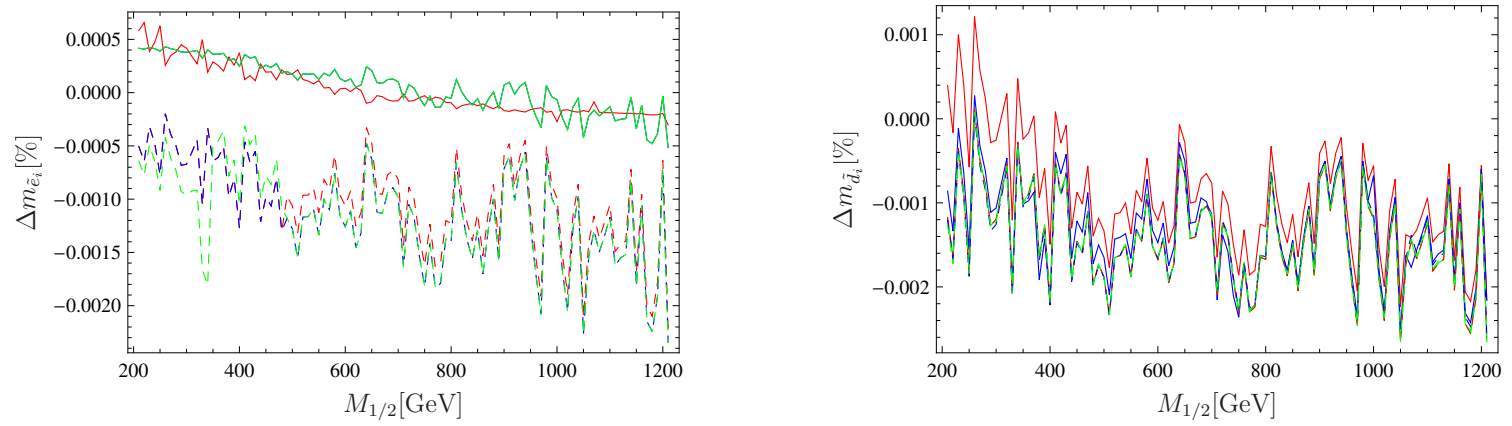

Figure 6: Comparison of the masses of all six charged sleptons and down squarks. Input values: $M_{0}=250 \mathrm{GeV}, A_{0}=-300 \mathrm{GeV}, \tan \beta=20, \operatorname{sign} \mu=1$.
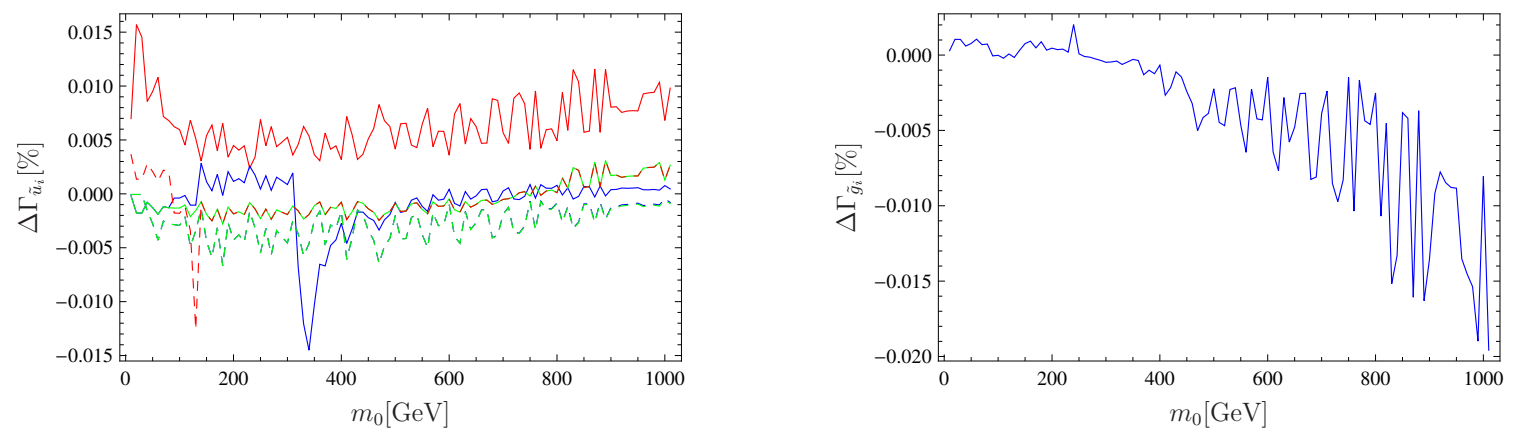

Figure 7: The dependence of the total decay widths of the up-squarks and the gluino on $m_{0}$. Input values: $M_{1 / 2}=500 \mathrm{GeV}, A_{0}=0 \mathrm{GeV}, \tan \beta=10$, sign $\mu=1$. As explained in the text, loop induced decays $\tilde{g} \rightarrow \tilde{\chi}^{0} g$ were disabled in SPheno "stock". 


\begin{tabular}{|l|cc|cc|cc|}
\hline & \multicolumn{2}{|c|}{$h_{1}$} & \multicolumn{2}{c|}{$h_{2}$} & \multicolumn{2}{c|}{$A^{0}$} \\
\hline$\Gamma[\mathrm{GeV}]$ & $2.21 \cdot 10^{-3}$ & $2.21 \cdot 10^{-3}$ & $7.30 \cdot 10^{-1}$ & $7.30 \cdot 10^{-1}$ & 1.16 & 1.16 \\
\hline \multicolumn{7}{|c|}{ Branching Ratios } \\
\hline$s \bar{s}$ & 0.0003 & 0.0003 & 0.0003 & 0.0003 & 0.0002 & 0.0002 \\
$b \bar{b}$ & 0.7763 & 0.7760 & 0.6402 & 0.6403 & 0.4034 & 0.4032 \\
$\mu \bar{\mu}$ & 0.0005 & 0.0005 & 0.0004 & 0.0004 & 0.0002 & 0.0002 \\
$\tau \bar{\tau}$ & 0.1287 & 0.1288 & 0.1051 & 0.1051 & 0.0662 & 0.0662 \\
$c \bar{c}$ & 0.0380 & 0.0379 & $<10^{-4}$ & $<10^{-4}$ & $<10^{-4}$ & $<10^{-4}$ \\
$t \bar{t}$ & - & - & 0.0667 & 0.0668 & 0.1224 & 0.1223 \\
$\tilde{\chi}_{1}^{-} \tilde{\chi}_{1}^{+}$ & - & - & 0.0400 & 0.0400 & 0.2010 & 0.2010 \\
$\tilde{\chi}_{1}^{0} \tilde{\chi}_{1}^{0}$ & - & - & 0.0234 & 0.0234 & 0.0230 & 0.0230 \\
$\tilde{\chi}_{1}^{0} \tilde{\chi}_{2}^{0}$ & - & - & 0.0643 & 0.0643 & 0.0962 & 0.0962 \\
$\tilde{\chi}_{2}^{0} \tilde{\chi}_{2}^{0}$ & - & - & 0.0145 & 0.0145 & 0.0758 & 0.0758 \\
$h_{1} h_{1}$ & - & - & 0.0143 & 0.0143 & - & - \\
$h_{1} Z$ & - & - & - & - & 0.0021 & 0.0021 \\
$\tilde{e}_{1}^{*} \tilde{e}_{1}$ & - & - & 0.0062 & 0.0062 & 0.0049 & 0.0048 \\
$\tilde{e}_{1}^{*} \tilde{e}_{6}$ & - & - & 0.0052 & 0.0052 & $<10^{-4}$ & $<10^{-4}$ \\
$\tilde{e}_{2}^{*} \tilde{e}_{2}$ & - & - & 0.0006 & 0.0006 & $<10^{-4}$ & $<10^{-4}$ \\
$\tilde{e}_{3}^{*} \tilde{e}_{3}$ & - & - & 0.0006 & 0.0006 & $<10^{-4}$ & $<10^{-4}$ \\
$\tilde{e}_{6}^{*} \tilde{e}_{1}$ & - & - & 0.0052 & 0.0052 & 0.0049 & 0.0048 \\
$\tilde{\nu}_{1}^{*} \tilde{\nu}_{1}$ & - & - & 0.0012 & 0.0012 & $<10^{-4}$ & $<10^{-4}$ \\
$\tilde{\nu}_{2}^{*} \tilde{\nu}_{2}$ & - & - & 0.0011 & 0.0011 & $<10^{-4}$ & $<10^{-4}$ \\
$\tilde{\nu}_{3}^{*} \tilde{\nu}_{3}$ & - & - & 0.0011 & 0.0011 & $<10^{-4}$ & $<10^{-4}$ \\
$\gamma \gamma$ & 0.0025 & 0.0025 & $<10^{-4}$ & $<10^{-4}$ & $<10^{-4}$ & $<10^{-4}$ \\
$g g$ & 0.0310 & 0.0309 & 0.0007 & 0.0007 & $<10^{-4}$ & $<10^{-4}$ \\
$Z Z^{*}$ & 0.0011 & 0.0010 & - & - & - & - \\
$Z Z$ & - & - & 0.0029 & 0.0029 & - & - \\
$W^{-}\left(W^{+}\right)^{*}$ & 0.0110 & 0.0110 & - & - & - & - \\
$\left(W^{-}\right)^{*} W^{+}$ & 0.0110 & 0.0110 & - & - & - & - \\
$W^{-} W^{+}$ & - & - & 0.0060 & 0.0060 & - & - \\
\hline
\end{tabular}

Table 4: Left column: SPheno/SARAH, right column: SPheno "stock". Input values: $m_{0}=$ $100 \mathrm{GeV}, M_{1 / 2}=250 \mathrm{GeV}, A_{0}=-100 \mathrm{GeV}, \tan \beta=10, \operatorname{sign} \mu=1$. 


\begin{tabular}{|l|cc|cc|cc|}
\hline & \multicolumn{3}{|c|}{$\tilde{\chi}_{2}^{0}$} & \multicolumn{2}{c|}{$\tilde{\chi}_{3}^{0}$} & \multicolumn{3}{c|}{$\tilde{\chi}_{4}^{0}$} \\
\hline$\Gamma[\mathrm{GeV}]$ & $6.57 \cdot 10^{-6}$ & $6.57 \cdot 10^{-6}$ & 1.39 & 1.39 & 1.60 & 1.60 \\
\hline \multicolumn{7}{|c|}{ Branching Ratios } \\
\hline$\tilde{\chi}_{1}^{0} d \bar{d}$ & 0.1742 & 0.1742 & $<10^{-4}$ & $<10^{-4}$ & $<10^{-4}$ & $<10^{-4}$ \\
$\tilde{\chi}_{1}^{0} s \bar{s}$ & 0.1742 & 0.1742 & $<10^{-4}$ & $<10^{-4}$ & $<10^{-4}$ & $<10^{-4}$ \\
$\tilde{\chi}_{1}^{0} b \bar{b}$ & 0.1762 & 0.1763 & $<10^{-4}$ & $<10^{-4}$ & $<10^{-4}$ & $<10^{-4}$ \\
$\tilde{\chi}_{1}^{0} e \bar{e}$ & 0.0227 & 0.0227 & $<10^{-4}$ & $<10^{-4}$ & $<10^{-4}$ & $<10^{-4}$ \\
$\tilde{\chi}_{1}^{0} \mu \bar{\mu}$ & 0.0227 & 0.0227 & $<10^{-4}$ & $<10^{-4}$ & $<10^{-4}$ & $<10^{-4}$ \\
$\tilde{\chi}_{1}^{0} \tau \bar{\tau}$ & 0.0230 & 0.0231 & $<10^{-4}$ & $<10^{-4}$ & $<10^{-4}$ & $<10^{-4}$ \\
$\tilde{\chi}_{1}^{0} u \bar{u}$ & 0.1293 & 0.1293 & $<10^{-4}$ & $<10^{-4}$ & $<10^{-4}$ & $<10^{-4}$ \\
$\tilde{\chi}_{1}^{0} c \bar{c}$ & 0.1289 & 0.1287 & $<10^{-4}$ & $<10^{-4}$ & $<10^{-4}$ & $<10^{-4}$ \\
$\tilde{\chi}_{1}^{0} \nu_{i} \bar{\nu}_{i}$ & 0.1487 & 0.1486 & $<10^{-4}$ & $<10^{-4}$ & $<10^{-4}$ & $<10^{-4}$ \\
$\tilde{\chi}_{1}^{-} W^{+}$ & - & - & 0.3137 & 0.3137 & 0.3519 & 0.3519 \\
$\tilde{\chi}_{1}^{+} W^{-}$ & - & - & 0.3137 & 0.3137 & 0.3519 & 0.3519 \\
$\tilde{\chi}_{1}^{0} h_{1}$ & - & - & 0.0269 & 0.0260 & 0.0904 & 0.0904 \\
$\tilde{\chi}_{2}^{0} h_{1}$ & - & - & 0.1228 & 0.1228 & 0.1467 & 0.1455 \\
$\tilde{\chi}_{1}^{0} Z$ & - & - & 0.1359 & 0.1359 & 0.0327 & 0.0327 \\
$\tilde{\chi}_{2}^{0} Z$ & - & - & 0.1985 & 0.1985 & 0.0264 & 0.0264 \\
\hline
\end{tabular}

Table 5: Left column: SPheno/SARAH, right column: SPheno "stock". Input values: $m_{0}=$ $550 \mathrm{GeV}, M_{1 / 2}=200 \mathrm{GeV}, A_{0}=-100 \mathrm{GeV}, \tan \beta=10, \operatorname{sign} \mu=1$. 


\begin{tabular}{|c|c|c|c|c|c|c|}
\hline & \multicolumn{2}{|c|}{ Seesaw I } & \multicolumn{2}{|c|}{ Seesaw II } & \multicolumn{2}{|c|}{ Seesaw III } \\
\hline particle & SPheno & SARAH & SPheno & SARAH & SPheno & SARAH \\
\hline$M_{\mathrm{GUT}}$ & $2.36 \cdot 10^{16}$ & $2.36 \cdot 10^{16}$ & $2.75 \cdot 10^{16}$ & $2.75 \cdot 10^{16}$ & $3.61 \cdot 10^{16}$ & $3.64 \cdot 10^{16}$ \\
\hline$\tilde{\chi}_{1}^{0}$ & 97.59 & 97.61 & 76.07 & 76.17 & 47.04 & 46.92 \\
\hline$\tilde{\chi}_{2}^{0}$ & 180.26 & 180.30 & 139.44 & 139.51 & 84.42 & 84.21 \\
\hline$\tilde{\chi}_{3}^{0}$ & 345.69 & 345.93 & 294.96 & 294.47 & 209.78 & 209.56 \\
\hline$\tilde{\chi}_{4}^{0}$ & 365.60 & 365.81 & 315.62 & 315.22 & 234.36 & 234.39 \\
\hline$\tilde{\chi}_{1}^{-}$ & 179.74 & 179.78 & 138.59 & 138.65 & 81.91 & 81.70 \\
\hline$\tilde{\chi}_{2}^{-}$ & 366.57 & 366.78 & 317.00 & 316.60 & 236.90 & 236.69 \\
\hline$\tilde{g}$ & 621.94 & 622.17 & 509.89 & 509.78 & 366.28 & 365.81 \\
\hline$h_{1}$ & 105.73 & 105.73 & 104.11 & 104.11 & 101.41 & 101.40 \\
\hline$h_{2}$ & 443.91 & 444.07 & 384.29 & 385.31 & 320.91 & 320.74 \\
\hline$A^{0}$ & 443.64 & 443.81 & 384.03 & 385.31 & 320.64 & 320.46 \\
\hline$H^{+}$ & 451.26 & 451.41 & 392.65 & 393.65 & 330.69 & 330.56 \\
\hline$\tilde{e}_{1}$ & 263.89 & 263.85 & 258.62 & 258.77 & 253.34 & 253.38 \\
\hline$\tilde{e}_{2}$ & 270.25 & 270.25 & 264.94 & 265.09 & 259.33 & 259.31 \\
\hline$\tilde{e}_{3}$ & 270.25 & 270.25 & 264.97 & 265.11 & 259.33 & 259.31 \\
\hline$\tilde{e}_{4}$ & 304.59 & 304.58 & 288.40 & 288.46 & 270.48 & 270.42 \\
\hline$\tilde{e}_{5}$ & 304.60 & 304.59 & 288.41 & 288.47 & 270.48 & 270.43 \\
\hline$\tilde{e}_{6}$ & 306.38 & 306.22 & 290.41 & 290.56 & 272.41 & 272.36 \\
\hline$\tilde{d}_{1}$ & 551.93 & 552.14 & 466.24 & 466.39 & 357.84 & 357.51 \\
\hline$\tilde{d}_{2}$ & 592.29 & 592.50 & 505.04 & 505.11 & 396.81 & 396.48 \\
\hline$\tilde{d}_{3}$ & 593.97 & 594.18 & 506.27 & 506.35 & 398.12 & 397.78 \\
\hline$\tilde{d}_{4}$ & 593.98 & 594.18 & 506.27 & 506.35 & 398.12 & 307.79 \\
\hline$\tilde{d}_{5}$ & 614.46 & 614.82 & 522.97 & 523.06 & 409.38 & 409.03 \\
\hline$\tilde{d}_{6}$ & 614.46 & 614.82 & 522.97 & 523.07 & 409.38 & 409.04 \\
\hline$\tilde{u}_{1}$ & 439.39 & 439.62 & 367.16 & 367.10 & 277.73 & 277.47 \\
\hline$\tilde{u}_{2}$ & 594.19 & 594.40 & 505.87 & 505.93 & 396.56 & 396.22 \\
\hline$\tilde{u}_{3}$ & 594.20 & 594.41 & 505.88 & 505.94 & 396.56 & 396.22 \\
\hline$\tilde{u}_{4}$ & 609.64 & 609.84 & 517.06 & 517.16 & 401.71 & 401.35 \\
\hline$\tilde{u}_{5}$ & 609.64 & 609.84 & 517.07 & 517.16 & 401.72 & 401.36 \\
\hline$\tilde{u}_{6}$ & 612.32 & 612.56 & 530.93 & 530.92 & 426.07 & 425.71 \\
\hline$\tilde{\nu}_{1}$ & 292.48 & 292.29 & 275.68 & 275.74 & 256.86 & 256.81 \\
\hline$\tilde{\nu}_{2}$ & 293.79 & 293.78 & 276.94 & 277.00 & 258.14 & 258.09 \\
\hline$\tilde{\nu}_{3}$ & 293.79 & 293.78 & 276.94 & 277.00 & 258.15 & 258.09 \\
\hline
\end{tabular}

Table 6: Comparison for Seesaw type I-III. Input values: $m_{0}=250 \mathrm{GeV}, M_{1 / 2}=250 \mathrm{GeV}$, $A_{0}=-0 \mathrm{GeV}, \tan \beta=10, \operatorname{sign} \mu=1$ and a (degenerated) Seesaw scale of $5 \cdot 10^{14} \mathrm{GeV}$ was used. 


\section{Validation of the WHIZARD model files}

We compared the WHIZARD model generated by SARAH from MSSM-NOFV to the MSSM implementation included in WHIZARD. The result is shown in Tab.7 for a selection of $2 \rightarrow 2$ processes and shows complete agreement between the two models.

For the comparison, the input parameters were chosen as defined by the following SINDARIN code:

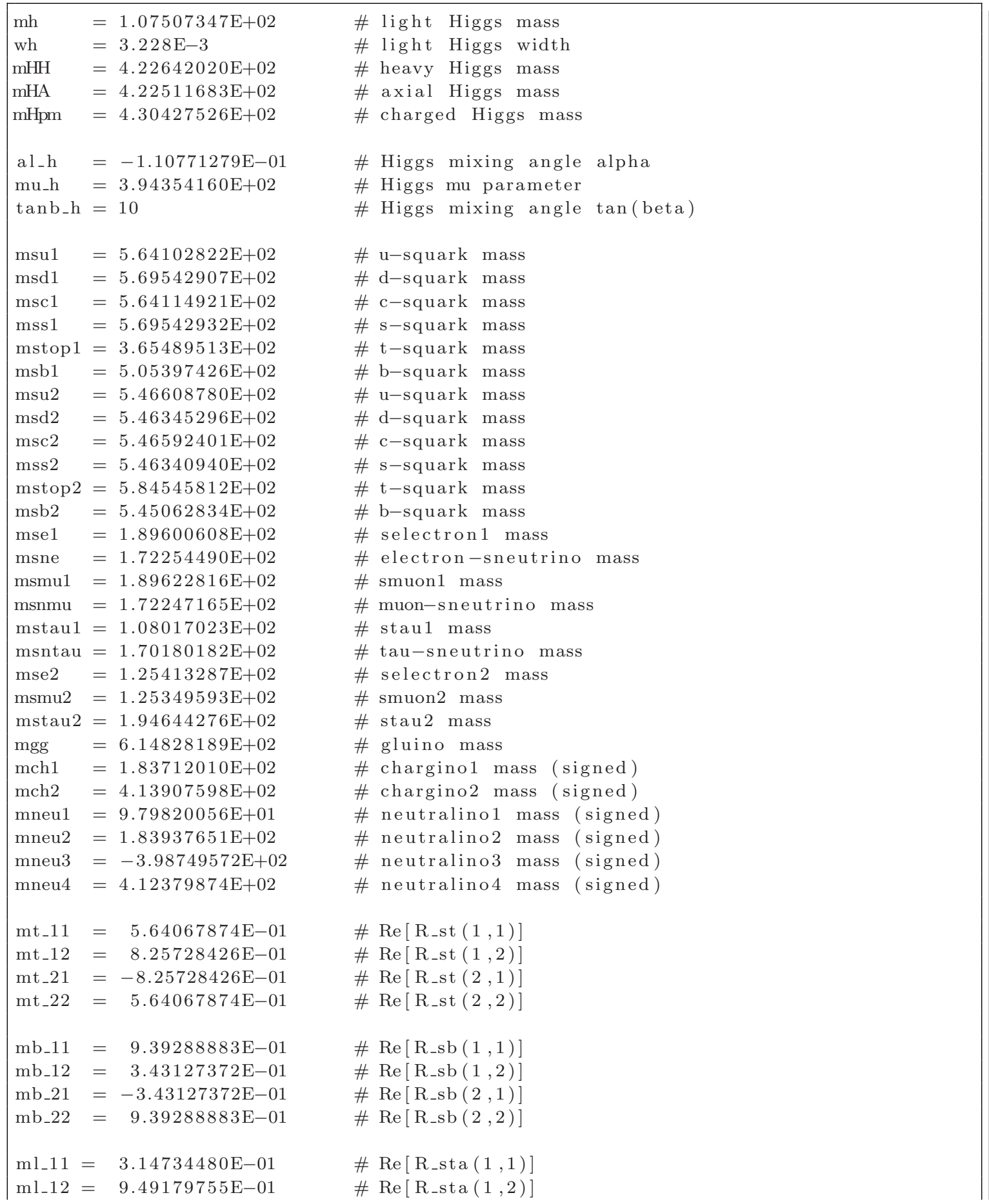




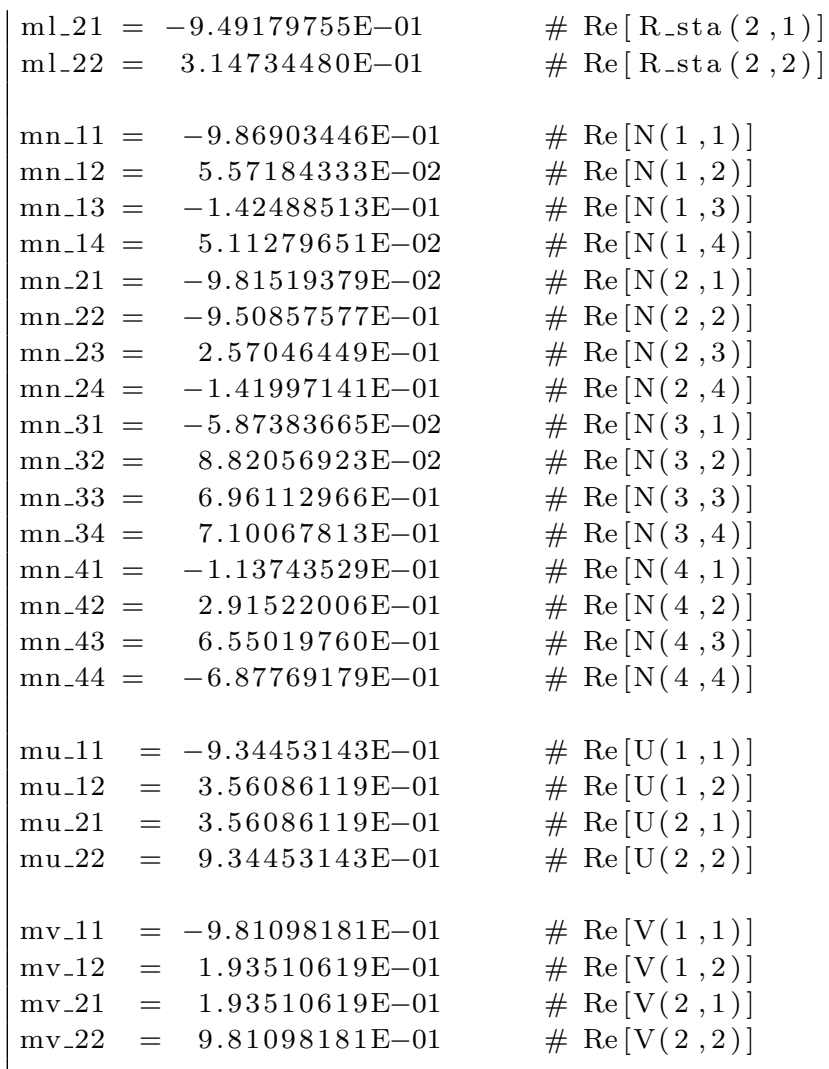

The intergration was performed for a CMS energy of $2 \mathrm{GeV}$, with cuts and integration parameters chosen as exemplified by the following sample input (with the obvious replacement of $I 1 / 2$ and $F 1 / F 2$ by the actual particle identifiers):

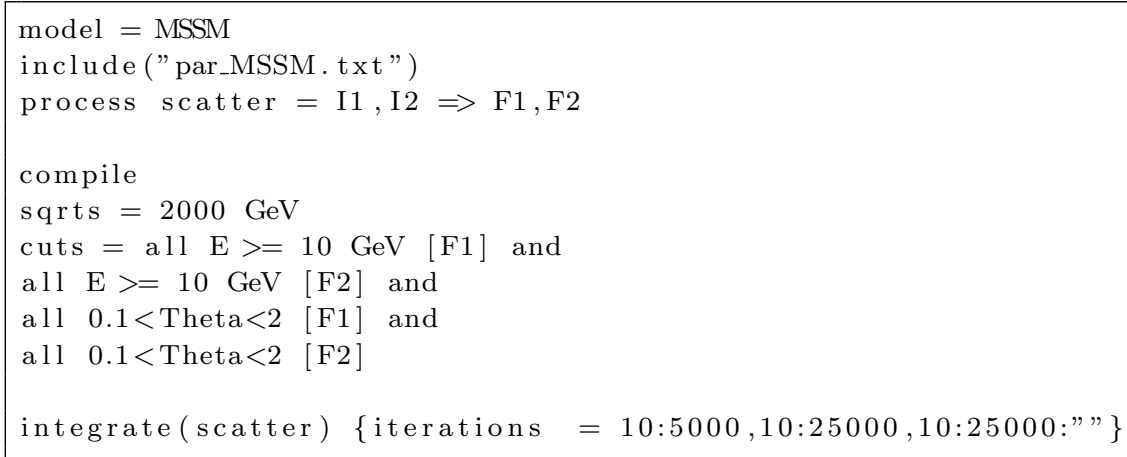




\begin{tabular}{|c|c|c|c|c|c|}
\hline Process & $\sigma_{S}[\mathrm{fb}]$ & $\delta_{S}[\mathrm{fb}]$ & $\sigma_{W}[\mathrm{fb}]$ & $\delta_{W}[\mathrm{fb}]$ & $\Delta[\mathrm{fb}]$ \\
\hline$\tilde{\chi}_{1}^{0} \tilde{\chi}_{1}^{0} \rightarrow Z Z$ & $1.227 \times 10^{-1}$ & $9.87 \times 10^{-6}$ & $1.227 \times 10^{-1}$ & $1.35 \times 10^{-5}$ & $3.28 \times 10^{-5}$ \\
\hline$\tilde{\chi}_{1}^{0} \tilde{\chi}_{1}^{0} \rightarrow \tilde{\chi}_{1}^{0} \tilde{\chi}_{1}^{0}$ & $2.538 \times 10^{-2}$ & $1.34 \times 10^{-6}$ & $2.538 \times 10^{-2}$ & $3.65 \times 10^{-6}$ & $-2.99 \times 10^{-6}$ \\
\hline$\tilde{\chi}_{1}^{0} \tilde{\chi}_{1}^{0} \rightarrow \tilde{\chi}_{2}^{0} \tilde{\chi}_{2}^{0}$ & $2.219 \times 10^{-1}$ & $1.14 \times 10^{-5}$ & $2.219 \times 10^{-1}$ & $1.03 \times 10^{-5}$ & $3.4 \times 10^{-6}$ \\
\hline$\tilde{\chi}_{1}^{0} \tilde{\chi}_{1}^{0} \rightarrow \tilde{\chi}_{3}^{0} \tilde{\chi}_{3}^{0}$ & 1.762 & $1.24 \times 10^{-4}$ & 1.762 & $1.24 \times 10^{-4}$ & $-2.7 \times 10^{-4}$ \\
\hline$\tilde{\chi}_{1}^{0} \tilde{\chi}_{1}^{0} \rightarrow \tilde{\chi}_{4}^{0} \tilde{\chi}_{4}^{0}$ & 1.105 & $8.23 \times 10^{-5}$ & 1.104 & $6.36 \times 10^{-5}$ & $1.37 \times 10^{-4}$ \\
\hline$\tilde{\chi}_{1}^{0} \tilde{\chi}_{1}^{0} \rightarrow h A^{0}$ & $3.913 \times 10^{-1}$ & $3.46 \times 10^{-5}$ & $3.913 \times 10^{-1}$ & $2.36 \times 10^{-5}$ & $1.43 \times 10^{-5}$ \\
\hline$\tilde{\chi}_{1}^{0} \tilde{\chi}_{1}^{0} \rightarrow H A^{0}$ & 1.843 & $2.10 \times 10^{-4}$ & 1.841 & $1.68 \times 10^{-4}$ & $1.38 \times 10^{-3}$ \\
\hline$\tilde{\chi}_{1}^{0} \tilde{\chi}_{1}^{0} \rightarrow \tilde{\chi}_{1}^{+} \tilde{\chi}_{1}^{-}$ & $1.069 \times 10^{-1}$ & $6.69 \times 10^{-6}$ & $1.068 \times 10^{-1}$ & $1.10 \times 10^{-5}$ & $4.44 \times 10^{-5}$ \\
\hline$\tilde{\chi}_{1}^{0} \tilde{\chi}_{1}^{0} \rightarrow \tilde{\chi}_{2}^{+} \tilde{\chi}_{2}^{-}$ & 1.542 & $1.69 \times 10^{-4}$ & 1.541 & $1.34 \times 10^{-4}$ & $1.19 \times 10^{-3}$ \\
\hline$\tilde{\chi}_{1}^{0} \tilde{\chi}_{1}^{0} \rightarrow \tilde{\chi}_{1}^{0} \tilde{\chi}_{2}^{0}$ & $1.504 \times 10^{-1}$ & $1.32 \times 10^{-5}$ & $1.504 \times 10^{-1}$ & $2.04 \times 10^{-5}$ & $-6.14 \times 10^{-5}$ \\
\hline$\tilde{\chi}_{1}^{0} \tilde{\chi}_{1}^{0} \rightarrow W^{-} W^{+}$ & 1.57 & $1.73 \times 10^{-4}$ & 1.57 & $1.55 \times 10^{-4}$ & $-5.73 \times 10^{-5}$ \\
\hline$\tilde{\chi}_{1}^{0} \tilde{\chi}_{1}^{0} \rightarrow d \bar{d}$ & $6.836 \times 10^{-1}$ & $8.44 \times 10^{-5}$ & $6.838 \times 10^{-1}$ & $5.21 \times 10^{-5}$ & $-2.12 \times 10^{-4}$ \\
\hline$\tilde{\chi}_{1}^{0} \tilde{\chi}_{1}^{0} \rightarrow t \bar{t}$ & $1.234 \times 10^{1}$ & $1.18 \times 10^{-3}$ & $1.234 \times 10^{1}$ & $8.07 \times 10^{-4}$ & $6.67 \times 10^{-4}$ \\
\hline$\tilde{\chi}_{1}^{0} \tilde{\chi}_{1}^{0} \rightarrow b \bar{b}$ & $7.878 \times 10^{-1}$ & $9.42 \times 10^{-5}$ & $7.879 \times 10^{-1}$ & $8.46 \times 10^{-5}$ & $-3.97 \times 10^{-5}$ \\
\hline$\tilde{\chi}_{1}^{0} \tilde{\chi}_{1}^{0} \rightarrow \tilde{t}_{1} \tilde{t}_{1}^{*}$ & 9.561 & $1.02 \times 10^{-3}$ & 9.563 & $8.33 \times 10^{-4}$ & $-2.5 \times 10^{-3}$ \\
\hline$\tilde{\chi}_{1}^{0} \tilde{\chi}_{1}^{0} \rightarrow \tilde{t}_{2} \tilde{t}_{2}^{*}$ & $5.421 \times 10^{-1}$ & $3.56 \times 10^{-5}$ & $5.421 \times 10^{-1}$ & $4.61 \times 10^{-5}$ & $6.33 \times 10^{-5}$ \\
\hline$\tilde{\chi}_{1}^{0} \tilde{\chi}_{1}^{0} \rightarrow \tilde{t}_{1} \tilde{t}_{2}^{*}$ & 2.096 & $1.88 \times 10^{-4}$ & 2.098 & $2.22 \times 10^{-4}$ & $-1.92 \times 10^{-3}$ \\
\hline$\tilde{\chi}_{1}^{0} \tilde{\chi}_{1}^{0} \rightarrow \tilde{t}_{2} \tilde{t}_{1}^{*}$ & 2.097 & $1.70 \times 10^{-4}$ & 2.097 & $1.63 \times 10^{-4}$ & $-3.18 \times 10^{-5}$ \\
\hline$\tilde{\chi}_{1}^{0} \tilde{\chi}_{1}^{0} \rightarrow \tilde{d}_{1} \tilde{d}_{1}^{*}$ & $1.844 \times 10^{-1}$ & $1.20 \times 10^{-5}$ & $1.843 \times 10^{-1}$ & $1.58 \times 10^{-5}$ & $6.5 \times 10^{-5}$ \\
\hline$\tilde{\chi}_{1}^{0} \tilde{\chi}_{1}^{0} \rightarrow \tilde{d}_{2} \tilde{d}_{2}^{*}$ & $7.987 \times 10^{-1}$ & $7.22 \times 10^{-5}$ & $7.986 \times 10^{-1}$ & $7.23 \times 10^{-5}$ & $1.01 \times 10^{-4}$ \\
\hline$\tilde{\chi}_{1}^{0} \tilde{\chi}_{1}^{0} \rightarrow \tilde{b}_{1} \tilde{b}_{2}^{*}$ & $2.059 \times 10^{-1}$ & $1.54 \times 10^{-5}$ & $2.058 \times 10^{-1}$ & $2.20 \times 10^{-5}$ & $3.32 \times 10^{-6}$ \\
\hline$\tilde{\chi}_{1}^{0} \tilde{\chi}_{1}^{0} \rightarrow \tilde{b}_{2} \tilde{b}_{1}^{*}$ & $2.057 \times 10^{-1}$ & $2.08 \times 10^{-5}$ & $2.06 \times 10^{-1}$ & $2.82 \times 10^{-5}$ & $-2.11 \times 10^{-4}$ \\
\hline$\tilde{\chi}_{1}^{0} \tilde{\chi}_{1}^{0} \rightarrow \nu_{e} \bar{\nu}_{e}$ & 2.209 & $2.56 \times 10^{-4}$ & 2.21 & $1.65 \times 10^{-4}$ & $-1.26 \times 10^{-3}$ \\
\hline$\tilde{\chi}_{1}^{0} \tilde{\chi}_{1}^{0} \rightarrow \nu_{\mu} \bar{\nu}_{\mu}$ & 2.211 & $1.59 \times 10^{-4}$ & 2.21 & $1.36 \times 10^{-4}$ & $6.83 \times 10^{-4}$ \\
\hline$\tilde{\chi}_{1}^{0} \tilde{\chi}_{1}^{0} \rightarrow \tilde{e}_{1} \tilde{e}_{1}^{*}$ & 1.074 & $7.01 \times 10^{-5}$ & 1.073 & $7.70 \times 10^{-5}$ & $2.87 \times 10^{-4}$ \\
\hline$\tilde{\chi}_{1}^{0} \tilde{\chi}_{1}^{0} \rightarrow \tilde{e}_{2} \tilde{e}_{2}^{*}$ & $2.605 \times 10^{1}$ & $2.79 \times 10^{-3}$ & $2.604 \times 10^{1}$ & $1.59 \times 10^{-3}$ & $9.78 \times 10^{-3}$ \\
\hline$\tilde{\chi}_{1}^{0} \tilde{\chi}_{1}^{0} \rightarrow \tilde{\tau}_{1} \tilde{\tau}_{1}^{*}$ & $2.148 \times 10^{1}$ & $1.47 \times 10^{-3}$ & $2.148 \times 10^{1}$ & $2.17 \times 10^{-3}$ & $5.8 \times 10^{-3}$ \\
\hline$\tilde{\chi}_{1}^{0} \tilde{\chi}_{1}^{0} \rightarrow \tilde{\tau}_{2} \tilde{\tau}_{2}$ & $4.171 \times 10^{-1}$ & $2.00 \times 10^{-4}$ & $4.169 \times 10^{-1}$ & $3.14 \times 10^{-5}$ & $1.9 \times 10^{-4}$ \\
\hline$\tilde{\chi}_{1}^{0} \tilde{\chi}_{1}^{0} \rightarrow \tilde{\tau}_{1} \tilde{\tau}_{2}$ & 2.64 & $2.34 \times 10^{-4}$ & 2.64 & $2.11 \times 10^{-4}$ & $5.81 \times 10^{-4}$ \\
\hline$\tilde{\chi}_{1}^{0} \tilde{\chi}_{1}^{0} \rightarrow \tilde{\tau}_{2} \tilde{\tau}_{1}^{*}$ & 2.639 & $1.95 \times 10^{-4}$ & 2.64 & $2.05 \times 10^{-4}$ & $-7.78 \times 10^{-4}$ \\
\hline$\tilde{\chi}_{1}^{0} \tilde{\chi}_{1}^{0} \rightarrow H^{-} H^{+}$ & 1.14 & $8.12 \times 10^{-5}$ & 1.14 & $1.00 \times 10^{-4}$ & $-4.47 \times 10^{-4}$ \\
\hline$\tilde{\chi}_{1}^{0} \tilde{\chi}_{1}^{0} \rightarrow h h$ & $7.951 \times 10^{-2}$ & $8.62 \times 10^{-6}$ & $7.948 \times 10^{-2}$ & $7.51 \times 10^{-6}$ & $2.98 \times 10^{-5}$ \\
\hline$\tilde{\chi}_{1}^{0} \tilde{\chi}_{1}^{0} \rightarrow H H$ & $5.380 \times 10^{-2}$ & $9.95 \times 10^{-6}$ & $5.380 \times 10^{-2}$ & $5.76 \times 10^{-6}$ & $3.25 \times 10^{-6}$ \\
\hline$\tilde{\chi}_{1}^{0} \tilde{\chi}_{1}^{0} \rightarrow h H$ & $2.643 \times 10^{-1}$ & $2.19 \times 10^{-5}$ & $2.643 \times 10^{-1}$ & $1.79 \times 10^{-5}$ & $8.54 \times 10^{-5}$ \\
\hline$\tilde{\chi}_{1}^{0} \tilde{\chi}_{1}^{0} \rightarrow A^{0} A^{0}$ & $5.487 \times 10^{-2}$ & $6.71 \times 10^{-6}$ & $5.492 \times 10^{-2}$ & $1.10 \times 10^{-5}$ & $-5.63 \times 10^{-5}$ \\
\hline$e \bar{e} \rightarrow Z Z$ & 2.325 & $2.42 \times 10^{-4}$ & 2.325 & $1.82 \times 10^{-4}$ & $-1.77 \times 10^{-4}$ \\
\hline$e \bar{e} \rightarrow \gamma \gamma$ & $1.636 \times 10^{1}$ & $1.52 \times 10^{-3}$ & $1.637 \times 10^{1}$ & $2.81 \times 10^{-3}$ & $-1.09 \times 10^{-2}$ \\
\hline$e \bar{e} \rightarrow \tilde{e}_{1} \tilde{e}_{1}^{*}$ & 5.823 & $4.33 \times 10^{-4}$ & 5.825 & $4.47 \times 10^{-4}$ & $-1.97 \times 10^{-3}$ \\
\hline$e \bar{e} \rightarrow \tilde{e}_{2} \tilde{e}_{2}^{*}$ & 4.955 & $6.10 \times 10^{-4}$ & 4.953 & $5.79 \times 10^{-4}$ & $1.74 \times 10^{-3}$ \\
\hline$e \bar{e} \rightarrow \tilde{\chi}_{1}^{0} \tilde{\chi}_{1}^{0}$ & $1.143 \times 10^{1}$ & $7.15 \times 10^{-4}$ & $1.143 \times 10^{1}$ & $1.01 \times 10^{-3}$ & $-9.57 \times 10^{-4}$ \\
\hline
\end{tabular}


Tab.7: continued

\begin{tabular}{|c|c|c|c|c|c|}
\hline Process & $\sigma_{S}[\mathrm{fb}]$ & $\delta_{S}[\mathrm{fb}]$ & $\sigma_{W}[\mathrm{fb}]$ & $\delta_{W}[\mathrm{fb}]$ & $\Delta[\mathrm{fb}]$ \\
\hline 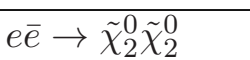 & 8.503 & $6.86 \times 10^{-4}$ & 8.503 & $6.09 \times 10^{-4}$ & $-9.71 \times 10^{-5}$ \\
\hline$e \bar{e} \rightarrow \tilde{\chi}_{3}^{0} \tilde{\chi}_{3}^{0}$ & $1.488 \times 10^{-3}$ & $1.37 \times 10^{-7}$ & $1.488 \times 10^{-3}$ & $1.01 \times 10^{-7}$ & $1.28 \times 10^{-7}$ \\
\hline$e \bar{e} \rightarrow \tilde{\chi}_{4}^{0} \tilde{\chi}_{4}^{0}$ & $4.235 \times 10^{-2}$ & $2.38 \times 10^{-6}$ & $4.234 \times 10^{-2}$ & $2.45 \times 10^{-6}$ & $8.23 \times 10^{-7}$ \\
\hline$e \bar{e} \rightarrow h A^{0}$ & $1.932 \times 10^{-4}$ & $1.41 \times 10^{-8}$ & $1.932 \times 10^{-4}$ & $3.38 \times 10^{-9}$ & $1.21 \times 10^{-8}$ \\
\hline$e \bar{e} \rightarrow H A^{0}$ & 1.351 & $1.06 \times 10^{-4}$ & 1.351 & $2.44 \times 10^{-5}$ & $-2.41 \times 10^{-4}$ \\
\hline$e \bar{e} \rightarrow \tilde{\chi}_{1}^{+} \tilde{\chi}_{1}^{-}$ & $1.658 \times 10^{1}$ & $1.25 \times 10^{-3}$ & $1.658 \times 10^{1}$ & $1.12 \times 10^{-3}$ & $6.14 \times 10^{-3}$ \\
\hline$e \bar{e} \rightarrow \tilde{\chi}_{2}^{+} \tilde{\chi}_{2}^{-}$ & 9.619 & $7.71 \times 10^{-4}$ & 9.617 & $5.87 \times 10^{-4}$ & $2.26 \times 10^{-3}$ \\
\hline$e \bar{e} \rightarrow \tilde{\chi}_{1}^{0} \tilde{\chi}_{2}^{0}$ & 4.361 & $5.20 \times 10^{-4}$ & 4.36 & $2.45 \times 10^{-4}$ & $1.14 \times 10^{-3}$ \\
\hline$e \bar{e} \rightarrow W^{-} W^{+}$ & $2.656 \times 10^{1}$ & $5.08 \times 10^{-3}$ & $2.657 \times 10^{1}$ & $1.50 \times 10^{-3}$ & $-1.21 \times 10^{-2}$ \\
\hline$e \bar{e} \rightarrow d \bar{d}$ & 7.563 & $8.01 \times 10^{-4}$ & 7.558 & $4.25 \times 10^{-4}$ & $5.42 \times 10^{-3}$ \\
\hline$e \bar{e} \rightarrow t \bar{t}$ & $1.428 \times 10^{1}$ & $1.58 \times 10^{-4}$ & $1.428 \times 10^{1}$ & $2.03 \times 10^{-3}$ & $-1.05 \times 10^{-3}$ \\
\hline$e \bar{e} \rightarrow b \bar{b}$ & 7.561 & $7.10 \times 10^{-4}$ & 7.559 & $4.15 \times 10^{-4}$ & $2.13 \times 10^{-3}$ \\
\hline$e \bar{e} \rightarrow \tilde{t}_{1} \tilde{t}_{1}^{*}$ & 3.695 & $1.15 \times 10^{-3}$ & 3.693 & $1.48 \times 10^{-4}$ & $1.92 \times 10^{-3}$ \\
\hline$e \bar{e} \rightarrow \tilde{t}_{2} \tilde{t}_{2}^{*}$ & 3.091 & $5.84 \times 10^{-5}$ & 3.087 & $3.37 \times 10^{-4}$ & $3.88 \times 10^{-3}$ \\
\hline$e \bar{e} \rightarrow \tilde{t}_{1} \tilde{t}_{2}^{*}$ & $7.906 \times 10^{-1}$ & $4.15 \times 10^{-6}$ & $7.906 \times 10^{-1}$ & $2.13 \times 10^{-6}$ & $-1.3 \times 10^{-5}$ \\
\hline$e \bar{e} \rightarrow \tilde{t}_{2} \tilde{t}_{1}^{*}$ & $7.907 \times 10^{-1}$ & $2.29 \times 10^{-5}$ & $7.907 \times 10^{-1}$ & $6.09 \times 10^{-6}$ & $6.73 \times 10^{-5}$ \\
\hline$e \bar{e} \rightarrow \tilde{d}_{1} \tilde{\tilde{d}}_{1}^{*}$ & 3.087 & $1.42 \times 10^{-5}$ & 3.086 & $1.09 \times 10^{-4}$ & $5.13 \times 10^{-4}$ \\
\hline$e \bar{e} \rightarrow \tilde{d}_{2} \tilde{d}_{2}^{*}$ & $6.913 \times 10^{-1}$ & $3.26 \times 10^{-6}$ & $6.913 \times 10^{-1}$ & $2.64 \times 10^{-6}$ & $-6.95 \times 10^{-7}$ \\
\hline$e \bar{e} \rightarrow \tilde{b}_{1} \tilde{b}_{2}^{*}$ & $3.482 \times 10^{-1}$ & $7.06 \times 10^{-5}$ & $3.484 \times 10^{-1}$ & $1.21 \times 10^{-6}$ & $-2.33 \times 10^{-4}$ \\
\hline$e \bar{e} \rightarrow \tilde{b}_{2} \tilde{b}_{1}^{*}$ & $3.482 \times 10^{-1}$ & $5.59 \times 10^{-5}$ & $3.484 \times 10^{-1}$ & $9.92 \times 10^{-6}$ & $-2.07 \times 10^{-4}$ \\
\hline$e \bar{e} \rightarrow \nu_{\mu} \bar{\nu}_{\mu}$ & 2.038 & $2.50 \times 10^{-5}$ & 2.038 & $1.49 \times 10^{-5}$ & $-2.14 \times 10^{-5}$ \\
\hline$e \bar{e} \rightarrow \tilde{\tau}_{1} \tilde{\tau}_{1}^{*}$ & 3.382 & $1.47 \times 10^{-5}$ & 3.383 & $1.07 \times 10^{-3}$ & $-1.55 \times 10^{-3}$ \\
\hline$e \bar{e} \rightarrow \tilde{\tau}_{2} \tilde{\tau}_{2}$ & 3.818 & $2.15 \times 10^{-4}$ & 3.816 & $1.73 \times 10^{-4}$ & $1.62 \times 10^{-3}$ \\
\hline$e \bar{e} \rightarrow \tilde{\tau}_{1} \tilde{\tau}_{2}$ & $1.56 \times 10^{-1}$ & $2.21 \times 10^{-6}$ & $1.56 \times 10^{-1}$ & $3.58 \times 10^{-6}$ & $3.54 \times 10^{-7}$ \\
\hline$e \bar{e} \rightarrow \tilde{\tau}_{2} \tilde{\tau}_{1}^{*}$ & $1.56 \times 10^{-1}$ & $2.12 \times 10^{-6}$ & $1.56 \times 10^{-1}$ & $1.38 \times 10^{-6}$ & $-5.22 \times 10^{-6}$ \\
\hline$e \bar{e} \rightarrow \gamma Z$ & $1.205 \times 10^{1}$ & $1.72 \times 10^{-3}$ & $1.204 \times 10^{1}$ & $8.95 \times 10^{-3}$ & $1.28 \times 10^{-2}$ \\
\hline$e \bar{e} \rightarrow H^{-} H^{+}$ & 3.148 & $1.85 \times 10^{-4}$ & 3.147 & $2.94 \times 10^{-5}$ & $1.63 \times 10^{-3}$ \\
\hline$\tau \bar{\tau} \rightarrow Z Z$ & 2.325 & $2.39 \times 10^{-4}$ & 2.325 & $1.67 \times 10^{-4}$ & $-4.3 \times 10^{-4}$ \\
\hline$\tau \bar{\tau} \rightarrow \gamma \gamma$ & $1.637 \times 10^{1}$ & $1.27 \times 10^{-3}$ & $1.637 \times 10^{1}$ & $1.26 \times 10^{-3}$ & $5.26 \times 10^{-3}$ \\
\hline$\tau \bar{\tau} \rightarrow \tilde{\chi}_{1}^{0} \tilde{\chi}_{1}^{0}$ & $1.149 \times 10^{1}$ & $1.43 \times 10^{-3}$ & $1.148 \times 10^{1}$ & $7.34 \times 10^{-4}$ & $4.91 \times 10^{-3}$ \\
\hline$\tau \bar{\tau} \rightarrow \tilde{\chi}_{2}^{0} \tilde{\chi}_{2}^{0}$ & 8.577 & $4.95 \times 10^{-4}$ & 8.576 & $6.90 \times 10^{-4}$ & $1.19 \times 10^{-3}$ \\
\hline$\tau \bar{\tau} \rightarrow \tilde{\chi}_{3}^{0} \tilde{\chi}_{3}^{0}$ & $1.805 \times 10^{-2}$ & $1.42 \times 10^{-6}$ & $1.805 \times 10^{-2}$ & $1.32 \times 10^{-6}$ & $-1.36 \times 10^{-6}$ \\
\hline$\tau \bar{\tau} \rightarrow \tilde{\chi}_{4}^{0} \tilde{\chi}_{4}^{0}$ & $8.165 \times 10^{-2}$ & $6.90 \times 10^{-6}$ & $8.161 \times 10^{-2}$ & $7.69 \times 10^{-6}$ & $3.49 \times 10^{-5}$ \\
\hline$\tau \bar{\tau} \rightarrow h A^{0}$ & $6.969 \times 10^{-4}$ & $7.26 \times 10^{-8}$ & $6.967 \times 10^{-4}$ & $1.02 \times 10^{-7}$ & $1.57 \times 10^{-7}$ \\
\hline$\tau \bar{\tau} \rightarrow H A^{0}$ & $9.225 \times 10^{-1}$ & $5.97 \times 10^{-5}$ & $9.223 \times 10^{-1}$ & $9.79 \times 10^{-5}$ & $1.57 \times 10^{-4}$ \\
\hline$\tau \bar{\tau} \rightarrow \tilde{\chi}_{1}^{+} \tilde{\chi}_{1}^{-}$ & $1.659 \times 10^{1}$ & $1.03 \times 10^{-3}$ & $1.659 \times 10^{1}$ & $1.23 \times 10^{-3}$ & $-2.02 \times 10^{-3}$ \\
\hline$\tau \bar{\tau} \rightarrow \tilde{\chi}_{2}^{+} \tilde{\chi}_{2}^{-}$ & 9.41 & $5.50 \times 10^{-4}$ & 9.412 & $1.01 \times 10^{-3}$ & $-1.6 \times 10^{-3}$ \\
\hline$\tau \bar{\tau} \rightarrow \tilde{\chi}_{1}^{0} \tilde{\chi}_{2}^{0}$ & 4.329 & $3.15 \times 10^{-4}$ & 4.326 & $3.45 \times 10^{-4}$ & $2.57 \times 10^{-3}$ \\
\hline$\tau \bar{\tau} \rightarrow W^{-} W^{+}$ & $2.66 \times 10^{1}$ & $1.98 \times 10^{-3}$ & $2.66 \times 10^{1}$ & $1.93 \times 10^{-3}$ & $-4.75 \times 10^{-3}$ \\
\hline$\tau \bar{\tau} \rightarrow d \bar{d}$ & 7.56 & $7.16 \times 10^{-4}$ & 7.56 & $4.47 \times 10^{-4}$ & $5.37 \times 10^{-4}$ \\
\hline
\end{tabular}


Tab.7: continued

\begin{tabular}{|c|c|c|c|c|c|}
\hline Process & $\sigma_{S}[\mathrm{fb}]$ & $\delta_{S}[\mathrm{fb}]$ & $\sigma_{W}[\mathrm{fb}]$ & $\delta_{W}[\mathrm{fb}]$ & $\Delta[\mathrm{fb}]$ \\
\hline$\overline{\tau \bar{\tau} \rightarrow t \bar{t}}$ & $1.428 \times 10^{1}$ & $5.20 \times 10^{-4}$ & $1.428 \times 10^{1}$ & $1.45 \times 10^{-4}$ & $-3.12 \times 10^{-3}$ \\
\hline$\tau \bar{\tau} \rightarrow b \bar{b}$ & 8.38 & $5.27 \times 10^{-4}$ & 8.377 & $4.90 \times 10^{-4}$ & $2.96 \times 10^{-3}$ \\
\hline$\tau \bar{\tau} \rightarrow \tilde{t}_{1} \tilde{t}_{1}^{*}$ & 3.804 & $1.07 \times 10^{-4}$ & 3.803 & $5.25 \times 10^{-4}$ & $4.57 \times 10^{-4}$ \\
\hline$\tau \bar{\tau} \rightarrow \tilde{t}_{2} \tilde{t}_{2}^{*}$ & 3.187 & $1.26 \times 10^{-5}$ & 3.185 & $7.57 \times 10^{-4}$ & $2.04 \times 10^{-3}$ \\
\hline$\tau \bar{\tau} \rightarrow \tilde{t}_{1} \tilde{t}_{2}^{*}$ & $9.566 \times 10^{-1}$ & $1.22 \times 10^{-4}$ & $9.564 \times 10^{-1}$ & $8.73 \times 10^{-5}$ & $1.72 \times 10^{-4}$ \\
\hline$\tau \bar{\tau} \rightarrow \tilde{t}_{2} \tilde{t}_{1}^{*}$ & $9.572 \times 10^{-1}$ & $1.80 \times 10^{-6}$ & $9.569 \times 10^{-1}$ & $4.59 \times 10^{-5}$ & $2.8 \times 10^{-4}$ \\
\hline$\tau \bar{\tau} \rightarrow \tilde{d}_{1} \tilde{d}_{1}^{*}$ & 3.087 & $7.22 \times 10^{-5}$ & 3.085 & $3.80 \times 10^{-5}$ & $1.29 \times 10^{-3}$ \\
\hline$\tau \bar{\tau} \rightarrow \tilde{d}_{2} \tilde{d}_{2}^{*}$ & $6.913 \times 10^{-1}$ & $2.90 \times 10^{-5}$ & $6.912 \times 10^{-1}$ & $4.63 \times 10^{-6}$ & $6.97 \times 10^{-5}$ \\
\hline$\tau \bar{\tau} \rightarrow \tilde{b}_{1} \tilde{b}_{2}^{*}$ & $3.484 \times 10^{-1}$ & $7.64 \times 10^{-7}$ & $3.485 \times 10^{-1}$ & $1.65 \times 10^{-5}$ & $-6.59 \times 10^{-5}$ \\
\hline$\tau \bar{\tau} \rightarrow \tilde{b}_{2} \tilde{b}_{1}^{*}$ & $3.48 \times 10^{-1}$ & $1.70 \times 10^{-4}$ & $3.484 \times 10^{-1}$ & $1.28 \times 10^{-6}$ & $-4.05 \times 10^{-4}$ \\
\hline$\tau \bar{\tau} \rightarrow \nu_{e} \bar{\nu}_{e}$ & 2.036 & $4.42 \times 10^{-4}$ & 2.038 & $8.12 \times 10^{-5}$ & $-1.22 \times 10^{-3}$ \\
\hline$\tau \bar{\tau} \rightarrow \nu_{\mu} \bar{\nu}_{\mu}$ & 2.038 & $7.67 \times 10^{-5}$ & 2.038 & $3.27 \times 10^{-5}$ & $-2.33 \times 10^{-4}$ \\
\hline$\tau \bar{\tau} \rightarrow \tilde{e}_{1} \tilde{e}_{1}^{*}$ & 4.052 & $5.98 \times 10^{-5}$ & 4.052 & $2.06 \times 10^{-4}$ & $-1.19 \times 10^{-4}$ \\
\hline$\tau \bar{\tau} \rightarrow \tilde{e}_{2} \tilde{e}_{2}^{*}$ & 3.444 & $3.51 \times 10^{-4}$ & 3.447 & $1.13 \times 10^{-5}$ & $-2.49 \times 10^{-3}$ \\
\hline$\tau \bar{\tau} \rightarrow \tilde{\tau}_{1} \tilde{\tau}_{1}^{*}$ & 3.454 & $1.92 \times 10^{-4}$ & 3.453 & $1.62 \times 10^{-4}$ & $1.39 \times 10^{-3}$ \\
\hline$\tau \bar{\tau} \rightarrow \tilde{\tau}_{2} \tilde{\tau}_{2}$ & 4.131 & $2.21 \times 10^{-4}$ & 4.131 & $1.98 \times 10^{-4}$ & $1.63 \times 10^{-4}$ \\
\hline$\tau \bar{\tau} \rightarrow \tilde{\tau}_{1} \tilde{\tau}_{2}$ & 1.426 & $1.08 \times 10^{-4}$ & 1.426 & $1.04 \times 10^{-4}$ & $-3.02 \times 10^{-5}$ \\
\hline$\tau \bar{\tau} \rightarrow \tilde{\tau}_{2} \tilde{\tau}_{1}^{*}$ & 1.425 & $9.58 \times 10^{-5}$ & 1.425 & $8.42 \times 10^{-5}$ & $2.39 \times 10^{-4}$ \\
\hline$\tau \bar{\tau} \rightarrow \gamma Z$ & $1.205 \times 10^{1}$ & $3.18 \times 10^{-3}$ & $1.206 \times 10^{1}$ & $2.10 \times 10^{-3}$ & $-4.33 \times 10^{-3}$ \\
\hline$\tau \bar{\tau} \rightarrow H^{-} H^{+}$ & 2.964 & $2.41 \times 10^{-4}$ & 2.963 & $3.02 \times 10^{-4}$ & $3.63 \times 10^{-4}$ \\
\hline$\tau \bar{\tau} \rightarrow h h$ & $3.539 \times 10^{-5}$ & $1.86 \times 10^{-9}$ & $3.540 \times 10^{-5}$ & $2.75 \times 10^{-9}$ & $-1.12 \times 10^{-8}$ \\
\hline$\tau \bar{\tau} \rightarrow H H$ & $1.317 \times 10^{-3}$ & $1.32 \times 10^{-7}$ & $1.316 \times 10^{-3}$ & $1.56 \times 10^{-7}$ & $6.19 \times 10^{-7}$ \\
\hline$\tau \bar{\tau} \rightarrow h H$ & $5.755 \times 10^{-4}$ & $5.17 \times 10^{-8}$ & $5.757 \times 10^{-4}$ & $6.97 \times 10^{-8}$ & $-1.80 \times 10^{-7}$ \\
\hline$\tau \bar{\tau} \rightarrow A^{0} A^{0}$ & $1.256 \times 10^{-3}$ & $1.65 \times 10^{-7}$ & $1.257 \times 10^{-3}$ & $3.07 \times 10^{-7}$ & $-5.60 \times 10^{-7}$ \\
\hline$d \bar{d} \rightarrow Z Z$ & 3.043 & $2.57 \times 10^{-4}$ & 3.042 & $1.50 \times 10^{-4}$ & $6.63 \times 10^{-4}$ \\
\hline$d \bar{d} \rightarrow \gamma \gamma$ & $6.740 \times 10^{-2}$ & $1.01 \times 10^{-5}$ & $6.733 \times 10^{-2}$ & $9.16 \times 10^{-6}$ & $6.95 \times 10^{-5}$ \\
\hline$d \bar{d} \rightarrow \tilde{\chi}_{1}^{0} \tilde{\chi}_{1}^{0}$ & $3.762 \times 10^{-2}$ & $2.54 \times 10^{-6}$ & $3.763 \times 10^{-2}$ & $3.39 \times 10^{-6}$ & $-9.14 \times 10^{-6}$ \\
\hline$d \bar{d} \rightarrow \tilde{\chi}_{2}^{0} \tilde{\chi}_{2}^{0}$ & 1.547 & $1.24 \times 10^{-4}$ & 1.547 & $1.91 \times 10^{-4}$ & $5.74 \times 10^{-4}$ \\
\hline$d \bar{d} \rightarrow \tilde{\chi}_{3}^{0} \tilde{\chi}_{3}^{0}$ & $9.423 \times 10^{-4}$ & $7.59 \times 10^{-8}$ & $9.424 \times 10^{-4}$ & $6.25 \times 10^{-8}$ & $-4.87 \times 10^{-8}$ \\
\hline$d \bar{d} \rightarrow \tilde{\chi}_{4}^{0} \tilde{\chi}_{4}^{0}$ & $2.998 \times 10^{-2}$ & $2.27 \times 10^{-6}$ & $3.000 \times 10^{-2}$ & $3.31 \times 10^{-6}$ & $-1.77 \times 10^{-5}$ \\
\hline$d \bar{d} \rightarrow h A^{0}$ & $9.509 \times 10^{-5}$ & $7.01 \times 10^{-9}$ & $9.514 \times 10^{-5}$ & $1.83 \times 10^{-8}$ & $-4.92 \times 10^{-8}$ \\
\hline$d \bar{d} \rightarrow H A^{0}$ & $6.653 \times 10^{-1}$ & $3.82 \times 10^{-5}$ & $6.653 \times 10^{-1}$ & $1.87 \times 10^{-6}$ & $-3.64 \times 10^{-6}$ \\
\hline$d \bar{d} \rightarrow \tilde{\chi}_{1}^{+} \tilde{\chi}_{1}^{-}$ & 4.149 & $2.53 \times 10^{-4}$ & 4.148 & $2.74 \times 10^{-4}$ & $8.91 \times 10^{-4}$ \\
\hline$d \bar{d} \rightarrow \tilde{\chi}_{2}^{+} \tilde{\chi}_{2}^{-}$ & 1.532 & $9.89 \times 10^{-5}$ & 1.532 & $1.02 \times 10^{-4}$ & $2.56 \times 10^{-4}$ \\
\hline$d \bar{d} \rightarrow \tilde{\chi}_{1}^{0} \tilde{\chi}_{2}^{0}$ & $1.732 \times 10^{-1}$ & $1.20 \times 10^{-5}$ & $1.731 \times 10^{-1}$ & $1.33 \times 10^{-5}$ & $3.95 \times 10^{-5}$ \\
\hline$d \bar{d} \rightarrow W^{-} W^{+}$ & 8.039 & $8.13 \times 10^{-4}$ & 8.037 & $8.19 \times 10^{-4}$ & $1.94 \times 10^{-3}$ \\
\hline$d \bar{d} \rightarrow d \bar{d}$ & $5.63 \times 10^{3}$ & $5.52 \times 10^{-1}$ & $5.63 \times 10^{3}$ & $3.47 \times 10^{-1}$ & $-1.87 \times 10^{-2}$ \\
\hline$d \bar{d} \rightarrow t \bar{t}$ & $4.218 \times 10^{2}$ & $5.85 \times 10^{-2}$ & $4.222 \times 10^{2}$ & $5.25 \times 10^{-3}$ & $-4.27 \times 10^{-1}$ \\
\hline$d \bar{d} \rightarrow b \bar{b}$ & $4.179 \times 10^{2}$ & $3.76 \times 10^{-2}$ & $4.176 \times 10^{2}$ & $8.24 \times 10^{-2}$ & $3.12 \times 10^{-1}$ \\
\hline$d \bar{d} \rightarrow \tilde{t}_{1} \tilde{t}_{1}^{*}$ & $1.493 \times 10^{2}$ & $2.27 \times 10^{-3}$ & $1.493 \times 10^{2}$ & $6.30 \times 10^{-3}$ & $2.93 \times 10^{-2}$ \\
\hline
\end{tabular}


Tab.7: continued

\begin{tabular}{|c|c|c|c|c|c|}
\hline Process & $\sigma_{S}[\mathrm{fb}]$ & $\delta_{S}[\mathrm{fb}]$ & $\sigma_{W}[\mathrm{fb}]$ & $\delta_{W}[\mathrm{fb}]$ & $\Delta[\mathrm{fb}]$ \\
\hline$d \bar{d} \rightarrow \tilde{t}_{2} \tilde{t}_{2}^{*}$ & $9.922 \times 10^{1}$ & $1.34 \times 10^{-3}$ & $9.922 \times 10^{1}$ & $4.09 \times 10^{-4}$ & $-2.94 \times 10^{-3}$ \\
\hline$d \bar{d} \rightarrow \tilde{t}_{1} \tilde{t}_{2}^{*}$ & $3.893 \times 10^{-1}$ & $4.45 \times 10^{-6}$ & $3.893 \times 10^{-1}$ & $1.49 \times 10^{-4}$ & $-3.14 \times 10^{-6}$ \\
\hline$d \bar{d} \rightarrow \tilde{t}_{2} \tilde{t}_{1}^{*}$ & $3.893 \times 10^{-1}$ & $1.91 \times 10^{-5}$ & $3.892 \times 10^{-1}$ & $2.07 \times 10^{-6}$ & $2.72 \times 10^{-5}$ \\
\hline$d \bar{d} \rightarrow \tilde{d}_{1} \tilde{d}_{1}^{*}$ & $3.286 \times 10^{2}$ & $1.63 \times 10^{-2}$ & $3.286 \times 10^{2}$ & $2.04 \times 10^{-2}$ & $4.91 \times 10^{-2}$ \\
\hline$d \bar{d} \rightarrow \tilde{d}_{2} \tilde{d}_{2}^{*}$ & $3.973 \times 10^{2}$ & $2.08 \times 10^{-2}$ & $3.973 \times 10^{2}$ & $2.62 \times 10^{-2}$ & $2.6 \times 10^{-2}$ \\
\hline$d \bar{d} \rightarrow \tilde{b}_{1} \tilde{b}_{2}^{*}$ & $1.713 \times 10^{-1}$ & $1.58 \times 10^{-6}$ & $1.715 \times 10^{-1}$ & $1.97 \times 10^{-6}$ & $-2.02 \times 10^{-4}$ \\
\hline$d \bar{d} \rightarrow \tilde{b}_{2} \tilde{b}_{1}^{*}$ & $1.715 \times 10^{-1}$ & $2.92 \times 10^{-6}$ & $1.715 \times 10^{-1}$ & $6.87 \times 10^{-6}$ & $-1.12 \times 10^{-5}$ \\
\hline$d \bar{d} \rightarrow \nu_{e} \bar{\nu}_{e}$ & 1.003 & $4.84 \times 10^{-5}$ & 1.002 & $6.54 \times 10^{-4}$ & $1.22 \times 10^{-3}$ \\
\hline$d \bar{d} \rightarrow \nu_{\mu} \bar{\nu}_{\mu}$ & 1.003 & $1.03 \times 10^{-4}$ & 1.003 & $1.63 \times 10^{-4}$ & $5.07 \times 10^{-4}$ \\
\hline$d \bar{d} \rightarrow \tilde{e}_{1} \tilde{e}_{1}^{*}$ & $5.843 \times 10^{-1}$ & $4.91 \times 10^{-6}$ & $5.838 \times 10^{-1}$ & $1.38 \times 10^{-5}$ & $4.89 \times 10^{-4}$ \\
\hline$d \bar{d} \rightarrow \tilde{e}_{2} \tilde{e}_{2}^{*}$ & $1.28 \times 10^{-1}$ & $4.74 \times 10^{-6}$ & $1.28 \times 10^{-1}$ & $3.03 \times 10^{-6}$ & $1.72 \times 10^{-5}$ \\
\hline$d \bar{d} \rightarrow \tilde{\tau}_{1} \tilde{\tau}_{1}^{*}$ & $9.762 \times 10^{-2}$ & $3.05 \times 10^{-5}$ & $9.772 \times 10^{-2}$ & $3.57 \times 10^{-7}$ & $-1.01 \times 10^{-4}$ \\
\hline$d \bar{d} \rightarrow \tilde{\tau}_{2} \tilde{\tau}_{2}$ & $4.616 \times 10^{-1}$ & $4.36 \times 10^{-5}$ & $4.617 \times 10^{-1}$ & $7.55 \times 10^{-5}$ & $-1.35 \times 10^{-4}$ \\
\hline$d \bar{d} \rightarrow \tilde{\tau}_{1} \tilde{\tau}_{2}$ & $7.682 \times 10^{-2}$ & $9.73 \times 10^{-7}$ & $7.682 \times 10^{-2}$ & $4.04 \times 10^{-7}$ & $-3.85 \times 10^{-6}$ \\
\hline$d \bar{d} \rightarrow \tilde{\tau}_{2} \tilde{\tau}_{1}^{*}$ & $7.679 \times 10^{-2}$ & $7.09 \times 10^{-6}$ & $7.683 \times 10^{-2}$ & $2.60 \times 10^{-6}$ & $-4.29 \times 10^{-5}$ \\
\hline$d \bar{d} \rightarrow \gamma Z$ & $6.594 \times 10^{-1}$ & $5.08 \times 10^{-5}$ & $6.592 \times 10^{-1}$ & $4.78 \times 10^{-5}$ & $1.83 \times 10^{-4}$ \\
\hline$d \bar{d} \rightarrow H^{-} H^{+}$ & $4.539 \times 10^{-1}$ & $2.27 \times 10^{-5}$ & $4.539 \times 10^{-1}$ & $7.42 \times 10^{-6}$ & $-6.51 \times 10^{-5}$ \\
\hline$d \bar{d} \rightarrow \tilde{g} \tilde{g}$ & $4.938 \times 10^{2}$ & $2.83 \times 10^{-2}$ & $4.94 \times 10^{2}$ & $4.32 \times 10^{-2}$ & $-2.43 \times 10^{-1}$ \\
\hline$d \bar{d} \rightarrow g g$ & $1.118 \times 10^{3}$ & $1.03 \times 10^{-1}$ & $1.118 \times 10^{3}$ & $7.46 \times 10^{-2}$ & $-1.83 \times 10^{-1}$ \\
\hline$\gamma \gamma \rightarrow \tilde{\chi}_{1}^{+} \tilde{\chi}_{1}^{-}$ & $3.402 \times 10^{1}$ & $4.19 \times 10^{-3}$ & $3.401 \times 10^{1}$ & $2.50 \times 10^{-3}$ & 6. $\times 10^{-3}$ \\
\hline$\gamma \gamma \rightarrow W^{-} W^{+}$ & $3.22 \times 10^{2}$ & $2.21 \times 10^{-2}$ & $3.22 \times 10^{2}$ & $3.20 \times 10^{-2}$ & $5.72 \times 10^{-2}$ \\
\hline$\gamma \gamma \rightarrow \tilde{\chi}_{2}^{+} \tilde{\chi}_{2}^{-}$ & $3.696 \times 10^{1}$ & $2.23 \times 10^{-3}$ & $3.696 \times 10^{1}$ & $2.88 \times 10^{-3}$ & $4.95 \times 10^{-3}$ \\
\hline$\gamma \gamma \rightarrow d \bar{d}$ & 1.212 & $1.75 \times 10^{-4}$ & 1.213 & $2.79 \times 10^{-4}$ & $-6.44 \times 10^{-4}$ \\
\hline$\gamma \gamma \rightarrow t \bar{t}$ & $2.008 \times 10^{1}$ & $3.19 \times 10^{-3}$ & $2.009 \times 10^{1}$ & $2.72 \times 10^{-3}$ & $-8.75 \times 10^{-3}$ \\
\hline$\gamma \gamma \rightarrow b \bar{b}$ & 1.213 & $1.69 \times 10^{-4}$ & 1.213 & $9.21 \times 10^{-5}$ & $1.13 \times 10^{-4}$ \\
\hline$\gamma \gamma \rightarrow \tilde{t}_{1} \tilde{t}_{1}^{*}$ & 6.059 & $6.91 \times 10^{-4}$ & 6.058 & $4.91 \times 10^{-4}$ & $7.36 \times 10^{-4}$ \\
\hline$\gamma \gamma \rightarrow \tilde{t}_{2} \tilde{t}_{2}^{*}$ & 3.777 & $3.17 \times 10^{-4}$ & 3.778 & $3.37 \times 10^{-4}$ & $-3.11 \times 10^{-4}$ \\
\hline$\gamma \gamma \rightarrow \tilde{d}_{1} \tilde{\tilde{d}}_{1}^{*}$ & $2.44 \times 10^{-1}$ & $1.57 \times 10^{-5}$ & $2.44 \times 10^{-1}$ & $1.55 \times 10^{-5}$ & $5.97 \times 10^{-6}$ \\
\hline$\gamma \gamma \rightarrow \tilde{d}_{2} \tilde{d}_{2}^{*}$ & $2.57 \times 10^{-1}$ & $1.99 \times 10^{-5}$ & $2.569 \times 10^{-1}$ & $1.54 \times 10^{-5}$ & $7.42 \times 10^{-5}$ \\
\hline$\gamma \gamma \rightarrow \tilde{e}_{1} \tilde{e}_{1}^{*}$ & $1.319 \times 10^{1}$ & $8.09 \times 10^{-4}$ & $1.319 \times 10^{1}$ & $9.91 \times 10^{-4}$ & $1.09 \times 10^{-3}$ \\
\hline$\gamma \gamma \rightarrow \tilde{e}_{2} \tilde{e}_{2}^{*}$ & $1.391 \times 10^{1}$ & $7.59 \times 10^{-4}$ & $1.391 \times 10^{1}$ & $1.32 \times 10^{-3}$ & $-2.98 \times 10^{-4}$ \\
\hline$\gamma \gamma \rightarrow \tilde{\tau}_{1} \tilde{\tau}_{1}^{*}$ & $1.406 \times 10^{1}$ & $1.52 \times 10^{-3}$ & $1.406 \times 10^{1}$ & $1.13 \times 10^{-3}$ & $-6.26 \times 10^{-3}$ \\
\hline$\gamma \gamma \rightarrow \tilde{\tau}_{2} \tilde{\tau}_{2}$ & $1.312 \times 10^{1}$ & $6.91 \times 10^{-4}$ & $1.312 \times 10^{1}$ & $1.17 \times 10^{-3}$ & $1.12 \times 10^{-3}$ \\
\hline$\gamma \gamma \rightarrow H^{-} H^{+}$ & 8.983 & $1.07 \times 10^{-3}$ & 8.978 & $8.81 \times 10^{-4}$ & $4.25 \times 10^{-3}$ \\
\hline
\end{tabular}

Table 7: Comparision between the WHIZARD stock MSSM and the version generated by SARAH for several for various $2 \rightarrow 2$ processes. The calculated values for the cross sections are shown together with the respective absolute integration errors. See the text for details. 


\section{References}

[1] S. Weinberg, Phys. Rev. D 13 (1976) 974.

[2] S. Weinberg, Phys. Rev. D 19 (1979) 1277.

[3] P. Ramond, Phys. Rev. D 3 (1971) 2415.

[4] J. Wess and B. Zumino, Nucl. Phys. B 70 (1974) 39.

[5] D. V. Volkov and V. P. Akulov, Phys. Lett. B 46 (1973) 109.

[6] K. Inoue, A. Kakuto, H. Komatsu and S. Takeshita, Prog. Theor. Phys. 68 (1982) 927 [Erratum-ibid. 70 (1983) 330] [Prog. Theor. Phys. 70 (1983) 330].

[7] H. E. Haber and G. L. Kane, Phys. Rept. 117 (1985) 75.

[8] A. Djouadi et al. [MSSM Working Group], arXiv:hep-ph/9901246.

[9] H. Goldberg, Phys. Rev. Lett. 50 (1983) 1419 [Erratum-ibid. 103 (2009) 099905].

[10] J. R. Ellis, J. S. Hagelin, D. V. Nanopoulos, K. A. Olive and M. Srednicki, Nucl. Phys. B 238 (1984) 453.

[11] P. Langacker and M. x. Luo, Phys. Rev. D 44 (1991) 817.

[12] J. R. Ellis, S. Kelley and D. V. Nanopoulos, Phys. Lett. B 260 (1991) 131.

[13] C. Giunti, C. W. Kim and U. W. Lee, Mod. Phys. Lett. A 6 (1991) 1745.

[14] L. J. Hall, Nucl. Phys. B 178 (1981) 75.

[15] S. P. Martin, Phys. Rev. D 65 (2002) 116003 [arXiv:hep-ph/0111209].

[16] L. E. Ibanez and G. G. Ross, Phys. Lett. B 110 (1982) 215.

[17] Y. Fukuda et al. [Super-Kamiokande Collaboration], Phys. Rev. Lett. 81 (1998) 1562 [arXiv:hep-ex/9807003].

[18] K. Eguchi et al. [KamLAND Collaboration], Phys. Rev. Lett. 90 (2003) 021802 [arXiv:hep-ex/0212021].

[19] Q. R. Ahmad et al. [SNO Collaboration], Phys. Rev. Lett. 89 (2002) 011301 [arXiv:nucl-ex/0204008].

[20] T. Schwetz, M. Tortola and J. W. F. Valle, New J. Phys. 13 (2011) 063004 [arXiv:1103.0734 [hep-ph]].

[21] P. Minkowski, Phys. Lett. B 67 (1977) 421.

[22] T. Yanagida, in KEK lectures, ed. O. Sawada and A. Sugamoto, KEK, 1979; M Gell-Mann, P Ramond, R. Slansky, in Supergravity, ed. P. van Niewenhuizen and D. Freedman (North Holland, 1979);

[23] R.N. Mohapatra and G. Senjanovic, Phys. Rev. Lett. 44912 (1980).

[24] J. Schechter and J. W. F. Valle, Phys. Rev. D 22, 2227 (1980).

[25] T. P. Cheng and L. F. Li, Phys. Rev. D 22, 2860 (1980).

[26] R. Foot, H. Lew, X. G. He and G. C. Joshi, Z. Phys. C 44, 441 (1989).

[27] S. Weinberg, Phys. Rev. Lett. 43 (1979) 1566. 
[28] S. Weinberg, Phys. Rev. D 22 (1980) 1694.

[29] E. Ma, Phys. Rev. Lett. 81 (1998) 1171 [arXiv:hep-ph/9805219].

[30] L. J. Hall and M. Suzuki, Nucl. Phys. B 231 (1984) 419.

[31] J. C. Romao, M. A. Diaz, M. Hirsch, W. Porod and J. W. F. Valle, Phys. Rev. D 61 (2000) 071703 [arXiv:hep-ph/9907499].

[32] M. Hirsch, M. A. Diaz, W. Porod, J. C. Romao and J. W. F. Valle, Phys. Rev. D 62 (2000) 113008 [Erratum-ibid. D 65 (2002) 119901] [arXiv:hep-ph/0004115].

[33] A. Dedes, S. Rimmer and J. Rosiek, JHEP 0608 (2006) 005 [arXiv:hep-ph/0603225].

[34] R. D. Peccei and H. R. Quinn, Phys. Rev. Lett. 38 (1977) 1440.

[35] L. Covi and J. E. Kim, New J. Phys. 11 (2009) 105003 [arXiv:0902.0769 [astro-ph.CO]].

[36] J. E. Kim and H. P. Nilles, Phys. Lett. B 138 (1984) 150.

[37] K. Nakamura et al. [ Particle Data Group Collaboration ], J. Phys. G G37 (2010) 075021.

[38] U. Ellwanger, C. Hugonie and A. M. Teixeira, Phys. Rept. 496 (2010) 1-77. [arXiv:0910.1785 [hep-ph]].

[39] F. Staub, arXiv:0806.0538 [hep-ph].

[40] F. Staub, arXiv:0909.2863 [hep-ph].

[41] F. Staub, arXiv:1002.0840 [hep-ph].

[42] W. Porod, Comput. Phys. Commun. 153 (2003) 275 [arXiv:hep-ph/0301101].

[43] W. Porod and F. Staub, arXiv:1104.1573 [hep-ph].

[44] E. E. Boos, M. N. Dubinin, V. A. Ilyin, A. E. Pukhov and V. I. Savrin, arXiv:hep-ph/9503280.

[45] A. Pukhov, arXiv:hep-ph/0412191.

[46] G. Belanger, F. Boudjema, A. Pukhov and A. Semenov, Comput. Phys. Commun. 176 (2007) 367 [arXiv:hep-ph/0607059].

[47] P. Bechtle, O. Brein, S. Heinemeyer, G. Weiglein and K. E. Williams, Comput. Phys. Commun. 181 (2010) 138 [arXiv:0811.4169 [hep-ph]].

[48] P. Bechtle, O. Brein, S. Heinemeyer, G. Weiglein and K. E. Williams, arXiv:1102.1898 [hep-ph].

[49] M. Moretti, T. Ohl and J. Reuter, arXiv:hep-ph/0102195.

[50] W. Kilian, T. Ohl and J. Reuter, arXiv:0708.4233 [hep-ph].

[51] S. P. Martin and M. T. Vaughn, Phys. Rev. D 50 (1994) 2282 [Erratum-ibid. D 78 (2008) 039903] [arXiv:hep-ph/9311340].

[52] R. Fonseca, M. Malinsky, W. Porod, F. Staub, [arXiv:1107.2670 [hep-ph]].

[53] D. M. Pierce, J. A. Bagger, K. T. Matchev and R. j. Zhang, Nucl. Phys. B 491 (1997) 3 [arXiv:hep-ph/9606211].

[54] M. Bruhnke, B. Herrmann, W. Porod, JHEP 1009 (2010) 006. [arXiv:1007.2100 [hep-ph]].

[55] F. Staub, W. Porod and B. Herrmann, JHEP 1010 (2010) 040 [arXiv:1007.4049 [hep-ph]]. 
[56] G. Degrassi, P. Slavich and F. Zwirner, Nucl. Phys. B 611 (2001) 403 [arXiv:hep-ph/0105096];

[57] A. Brignole, G. Degrassi, P. Slavich and F. Zwirner, Nucl. Phys. B 631 (2002) 195 [arXiv:hep-ph/0112177];

[58] A. Brignole, G. Degrassi, P. Slavich and F. Zwirner, Nucl. Phys. B 643 (2002) 79 [arXiv:hep-ph/0206101];

[59] A. Dedes and P. Slavich, Nucl. Phys. B 657 (2003) 333 [arXiv:hep-ph/0212132];

[60] B. C. Allanach et al.,JHEP 0409, 044 (2004) [arXiv:hep-ph/0406166].

[61] P. Z. Skands, B. C. Allanach, H. Baer, C. Balazs, G. Belanger, F. Boudjema, A. Djouadi, R. Godbole et al., JHEP 0407, 036 (2004). [hep-ph/0311123].

[62] B. C. Allanach et al., Comput. Phys. Commun. 180 (2009) 8 [arXiv:0801.0045 [hep-ph]].

[63] M. Spira, A. Djouadi, D. Graudenz and P. M. Zerwas, Nucl. Phys. B 453 (1995) 17 [arXiv:hep-ph/9504378].

[64] M. Drees, K. -i. Hikasa, Phys. Lett. B240 (1990) 455.

[65] A. Djouadi, M. Spira and P. M. Zerwas, Z. Phys. C 70 (1996) 427 [arXiv:hep-ph/9511344].

[66] G. F. Giudice and R. Rattazzi, Phys. Rept. 322 (1999) 419 [arXiv:hep-ph/9801271].

[67] G. Belanger, N. D. Christensen, A. Pukhov and A. Semenov, arXiv:1008.0181 [hep-ph].

[68] F. Maltoni, K. Paul, T. Stelzer, S. Willenbrock, Phys. Rev. D67 (2003) 014026. [hep-ph/0209271].

[69] T. Ohl, Comput. Phys. Commun. 120 (1999) 13-19. [hep-ph/9806432].

[70] N. D. Christensen, C. Duhr, Comput. Phys. Commun. 180, 1614-1641 (2009). [arXiv:0806.4194 [hep-ph]].

[71] N. D. Christensen, C. Duhr, B. Fuks, J. Reuter, C. Speckner, [arXiv:1010.3251 [hep-ph]].

[72] J. N. Esteves, M. Hirsch, W. Porod, J. C. Romao, F. Staub and A. Vicente, JHEP 1012 (2010) 077 [arXiv:1011.0348 [hep-ph]]. 